\title{
The cratering record, chronology and surface ages of (4) Vesta in comparison to smaller asteroids and the ages of HED meteorites
}

\author{
N. Schmedemann ${ }^{\text {a,*, }}$ T. Kneissl ${ }^{\text {a }}$, B.A. Ivanov ${ }^{\text {b }}$, G.G. Michael ${ }^{\text {a }}$, R.J. Wagner ${ }^{c}$, G. Neukum ${ }^{\text {a }}$, \\ O. Ruesch ${ }^{\mathrm{d}}$, H. Hiesinger ${ }^{\mathrm{d}}$, K. Krohn ${ }^{\mathrm{c}}$, T. Roatsch ${ }^{\mathrm{c}}$, F. Preusker ${ }^{\mathrm{c}}$, H. Sierks ${ }^{\mathrm{e}}$, R. Jaumann ${ }^{\text {c,a }}$, \\ V. Reddy ${ }^{\text {h}}$, A. Nathues ${ }^{\text {e }}$, S.H.G. Walter ${ }^{a}$, A. Neesemann ${ }^{a}$, C.A. Raymond ${ }^{\mathrm{f}}$, C.T. Russell ${ }^{\mathrm{g}}$ \\ ${ }^{a}$ Institute of Geological Sciences, Freie Universität Berlin, Berlin, Germany \\ ${ }^{\mathrm{b}}$ Institute of Dynamics of Geospheres, Moscow, Russia \\ ${ }^{\mathrm{c}}$ German Aerospace Center (DLR), Institute of Planetary Research, Berlin, Germany \\ d Institut für Planetologie, Westfälische Wilhelms-Universität, Münster, Germany \\ e Max Planck Institute for Solar System Research (MPS), Göttingen, Germany \\ ${ }^{\mathrm{f}}$ Jet Propulsion Laboratory, California Institute of Technology, Pasadena, CA 91109, USA \\ ${ }^{\mathrm{g}}$ Institute of Geophysics and Planetary Physics, University of California Los Angeles (UCLA), Los Angeles, CA 90095, USA \\ h Planetary Science Institute, Tucson, Arizona 85719, USA
}

\section{A R T I C L E I N F O}

Article history:

Received 31 December 2012

Received in revised form

1 April 2014

Accepted 5 April 2014

Available online 18 April 2014

Keywords:

Vesta

Crater retention ages

Cratering chronology

Crater scaling

Rheasilvia

Veneneia

\begin{abstract}
A B S T R A C T
We derived model functions for the crater production size-frequency distribution and chronology of the asteroids 951 Gaspra, 243 Ida, 21 Lutetia and 4 Vesta, based on a lunar-like crater production function and a lunar-like chronology with a smooth exponential decay in impact rate for the first $\sim 1 \mathrm{Ga}$ of Solar System history. For Gaspra, Ida and Lutetia we find surface ages roughly in agreement with published data. Using the same approach for Vesta leads to results with high correlation to Ar-Ar reset ages of HED meteorites, for which a strong dynamical and spectroscopic connection to Vesta has been found. In contrast to recently published young formation ages of the Rheasilvia and Veneneia basins of about 1 and $2 \mathrm{Ga}$, respectively, we find for Rheasilvia a formation age of $3.5 \pm 0.1 \mathrm{Ga}$ and for the Veneneia formation a lower limit of $3.7 \pm 0.1 \mathrm{Ga}$. For comparison we also give surface model ages for a preliminary version of a chronology (pers. comm. D.P. O'Brien) based on the Late Heavy Bombardment theory. Error bars presented in our work stem only from statistical analysis of measured crater distributions and do not include the uncertainty of the used chronology model.
\end{abstract}

(c) 2014 Elsevier Ltd. All rights reserved.

\section{Introduction}

The asteroid Main Belt is most likely the chief source region of impacting projectiles in the inner Solar System (Neukum, 1984;

*Corresponding to: Institute of Geological Sciences, Malteserstraße 74-100, Building D, Freie Universität Berlin, 12249 Berlin, Germany. Tel.: +49 30838 70516; fax: +493083870539

E-mail addresses: nico.schmedemann@fu-berlin.de (N. Schmedemann), thomas.kneissl@fu-berlin.de (T. Kneissl), boris_a_ivanov@mail.ru (B.A. Ivanov), gregory.michael@fu-berlin.de (G.G. Michael), roland.wagner@dlr.de (R.J. Wagner), gerhard.neukum@fu-berlin.de (G. Neukum),

ottaviano.ruesch@uni-muenster.de (O. Ruesch),

hiesinger@uni-muenster.de (H. Hiesinger), katrin.krohn@dlr.de (K. Krohn),

Thomas.Roatsch@dlr.de (T. Roatsch), Frank.Preusker@dlr.de (F. Preusker),

sierks@mps.mpg.de (H. Sierks), Ralf.Jaumann@dlr.de (R. Jaumann),

reddy@psi.edu (V. Reddy), nathues@mps.mpg.de (A. Nathues),

sebastian.walter@fu-berlin.de (S.H.G. Walter),

adrian.neesemann@fu-berlin.de (A. Neesemann),

carol.a.raymond@jpl.nasa.gov (C.A. Raymond), ctrussel@igpp.ucla.edu (C.T. Russell).
Neukum and Ivanov, 1994; Neukum et al., 2001; Hiesinger et al., 2002; O'Brien and Greenberg, 2005; Strom et al., 2005; Ivanov, 2008; Massironi et al., 2009). As asteroids are believed to be the remains of the main building blocks at least of the inner major planets (Weidenschilling, 1974, 1976, 1977; Spaute et al., 1991; Inaba et al., 2003; Guillot and Gautier, 2007; Raymond et al., 2009; Weidenschilling, 2011) and thus also of the Earth (Alexander et al., 2012), it is of great interest for a number of planet and Solar System-related science topics to understand the diversity, nature and interaction of these bodies in a region, probably very similar to the very early Solar System. For this reason the first spacecraft dedicated to investigating an asteroid was christened "Dawn" (Russell et al., 2012). The Dawn mission will also investigate the only dwarf planet in the asteroid Main Belt, 1 Ceres (Russell et al., 2007; Russell and Raymond, 2011). Previous spacecraft had only investigated asteroidal bodies much smaller than Vesta (Table 1).

In this work we will review earlier work on asteroidal surface ages (Chapman et al., 1996a; Chapman et al., 1996b; Marchi et al., 
Table 1

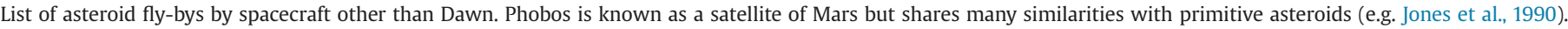

\begin{tabular}{|c|c|c|c|c|}
\hline Listed no. & Name & $\mathrm{s} / \mathrm{c}$ & Min. distance $[\mathrm{km}]$ & Date \\
\hline 5535 & Annefrank & Stardust & 3079 & 02.11 .2002 \\
\hline 132,524 & APL & New Horizons & 101,867 & 13.06.2006 \\
\hline 9969 & Braille & Deep Space 1 & 26 & 29.07.1999 \\
\hline 433 & Eros & NEAR Shoemaker & Landed & 12.02 .2001 \\
\hline 951 & Gaspra & Galileo & 1600 & 29.10.1991 \\
\hline 243 & Ida & Galileo & 2390 & 28.06 .1993 \\
\hline 25,143 & Itokawa & Hayabusa & Landed & 20./25.11.2005 \\
\hline 21 & Lutetia & Rosetta & 3170 & 10.07.2010 \\
\hline 2685 & Masursky & Cassini & $1,600,000$ & 23.01.2000 \\
\hline 253 & Mathilde & NEAR Shoemaker & 1212 & 27.06.1997 \\
\hline 2867 & Steins & Rosetta & 800 & 05.09 .2008 \\
\hline- & Phobos (may be captured) & $\begin{array}{l}\text { Mariner 9, Viking 1, Mars Global Surveyor, } \\
\text { Mars Express, Mars Reconnaissance Orbiter }\end{array}$ & 45 & 29.12.2013 (Mars Express) \\
\hline
\end{tabular}

$2012 \mathrm{~b}$ ) in order to compare the crater distributions and chronologies of small Main Belt asteroids such as 243 Ida, 951 Gaspra and 21 Lutetia with the much larger asteroid 4 Vesta. We will derive a chronology for each of these bodies and characterize the main features of their impact histories. Data on the smaller asteroids is predominantly presented in the supplementary online material of this paper.

Vesta is the chief source for basaltic HED (Howardite - Eucrite Diogenite) meteorites (McCord et al., 1970; Binzel and Xu, 1993; Moskovitz et al., 2008, 2010; McSween et al., 2011), although there is evidence for more sources of basaltic meteorites than just Vesta (Moskovitz et al., 2008; Roig et al., 2008; Scott et al., 2009). While earlier investigations of the cratering age of asteroids could not be cross-checked with meteorites for several reasons, radiometric ages of HED meteorites (e.g. Bogard and Garrison, 2003; Bogard, 2011) could be used to validate cratering chronology models of Vesta and thus, will have immediate consequences for earlier cratering age determinations of asteroids. These results will also have profound implications for understanding the dynamics and collisional history of the early inner Solar System. In general HED meteorites provide ground truth data for calibration and validity checks on Dawn science data.

\section{Methodology}

In order to derive absolute surface ages for asteroidal bodies, we measure crater frequencies and fit a crater production function to our measurements. From the fitted production function we determine the frequency of craters above a standard diameter, $1 \mathrm{~km}$. Knowing the frequency of craters $\geq 1 \mathrm{~km}$, we then use a chronology function to convert the measured crater frequency into a surface model age.

This technique is described in a number of papers (e.g. Neukum and Hiller, 1981; Neukum, 1984; Neukum and Ivanov, 1994; Michael and Neukum, 2010). For the calculation of surface ages, we use the "craterstats" software (Michael and Neukum, 2010). This software also allows for the determination of ages of partially resurfaced areas, for example by ejecta blanketing or seismic shaking.

Errors of Surface Model Ages: Errors given with model ages are derived from the size of the counting area and the number of craters used to fit the production function to the measured crater distribution. Due to the non-linear characteristics of the chronology functions for ages $>3 \mathrm{Ga}$, errors show some asymmetric characteristic (Michael and Neukum, 2010). Error bars for individual crater size bins in a crater plot represent the $1 \sigma$ standard deviation for the respective crater size bins (Crater Analysis Techniques Working Group et al., 1979). Such error bars are based on the number of craters within the individual crater bins. Thus, a bin with only one or a few craters has large error bars, while a bin with many craters has small error bars. The quoted errors give no measure of the likelihood of the used chronology model, the systematic errors of which could be much larger. Despite this, the ages may be interpreted in a relative sense with a level of confidence reflected by the quoted errors.

Coordinate System: The maps presented use the 'Claudia' system, which is the same system used in all publications of the Dawn results to date (Russell et al., 2012). The Planetary Data System provides data at different systems that follows IAU coordinate system recommendations (Archinal et al., 2011). The IAU longitudes are offset from the Claudia system by an addition of $150^{\circ}$.

Terminology: we use the term "lunar-like" to describe a chronology model which is scaled from the lunar model, but not to suggest that the Vesta chronology is the same as that of the Moon.

\subsection{Crater counting}

Crater counting is commonly performed on near spherical bodies such as the Moon, Mars, Mercury, Venus and the larger satellites of the giant planets in the outer Solar System. For this task we use the mapping software ArcGIS (ESRI) and the CraterTools plug-in (Kneissl et al., 2011), which simplifies crater counting. CraterTools allows for measuring crater size-frequency distributions on planetary surfaces independently of image and data frame map projections. All crater counts are performed by experienced human crater counters and cross checked by at least one other experienced crater counter. However, there are a number of specific sources of error, which are difficult to quantify such as the effect of solar illumination angles, photometric characteristics of images, and other similar factors. In addition, there is some variability in the identification of craters by different individuals. Each measurement is complemented with crater maps in the supplementary online material (SOM) chapter 1 (Ida, Gaspra and Lutetia; also measurement description) and chapter 2 (Vesta). We present our crater statistics following Crater Analysis Techniques Working Group et al. (1979), although we use a higher resolution pseudo-log binning with 18 intervals per decade (Neukum, 1984).

Due to the irregular shape of asteroids, data projection on a sphere introduces a source of error in spatial measurements. Therefore, we corrected our measurements gathered from projected imaging data according to a digital elevation model as described by Kneissl et al. (2014).

With increasing body size more ejecta are expected to be retained on the target body. On large asteroids ( $\varnothing>\sim 100 \mathrm{~km}$ ) with low to moderate porosity the surface gravity is sufficient to form well identifiable impact ejecta blankets (Housen and Holsapple, 2011, 
2012). For example, asteroids such as Vesta and even Lutetia are affected by resurfacing processes such as wide spread ejecta blanketing (Cintala et al., 1978) and also landslides occurring due to high relief accompanied by low surface gravity (Jaumann et al., 2012; Otto et al., 2013). To measure crater size-frequency distributions undisturbed by effects of an inhomogeneous geologic evolution, we mapped the counting areas inside identifiable geologic units as it is recommended by several publications (e.g. Baldwin, 1964; Neukum, 1984; Wilhelms et al., 1987; Michael and Neukum, 2010).

The effect of seismic shaking with variable surface gravity was investigated by Richardson (2013). It appears that a lower surface gravity leads to a broader spatial distribution of resurfacing effects following an impact. On large counting areas we therefore expect disturbed crater distributions due to multiple resurfacing events. Crater distributions on young ejecta blankets may be relatively undisturbed, because little time has passed in which other impacts could have caused resurfacing. On small asteroids such as Gaspra, the lower surface gravity and low escape velocity results in less ejecta blanketing, and no secondary craters can be formed (Cintala et al., 1978; Housen and Holsapple, 2011; Bierhaus et al., 2012). In the extreme case of $(25,143)$ Itokawa, there are almost no ejecta retained on its topographic highs, which show a barren rocky surface. Instead the fine grained material moved into the topographic lows (Mahaney et al., 2009).

Crater distributions affected by resurfacing events show a deficiency of small craters. That is because small craters are more easily degraded than larger ones. If a measured crater distribution shows obvious signs of such a resurfacing influence, only that part which appears yet to be intact is compared to the production function. Work on the effects of resurfacing has been done for example by Neukum and Hiller (1981), Hiesinger et al. (2002), Werner (2005), Hartmann and Werner (2010), Michael and Neukum (2010) and Fassett et al. (2012). It is possible to derive ages of resurfacing events: in the cumulative representation the craters larger than the fit range have to be discarded, which is done by the so-called "resurfacing correction" (Michael and Neukum, 2010).

Very old surfaces show crater frequencies so high that, on average, the formation of a new crater results in the obliteration of another crater of a similar size. Such a crater distribution has a shallower slope than the production crater distribution and described as being in equilibrium (Neukum and Dietzel, 1971; Neukum, 1984) or saturation (e.g. Hartmann, 1984; Richardson, 2009) for small craters ( $<\sim 10 \mathrm{~km}$ ). Larger craters usually follow a shallower production distribution, which does not change its shape even if the surface becomes saturated. That case is described with the term quasi-equilibrium (e.g. Neukum and Dietzel, 1971; Chapman et al., 1986). In both cases a surface age derived from such a crater distribution is always a minimum estimation of the true surface age. On a body with surface gravity lower than the Moon, more ejecta are lost to space and therefore less crater obliteration is expected. Thus, saturation may occur at a higher crater density.

\subsubsection{Spatial randomness analysis}

The bombardment of Vesta and other asteroids is expected to produce a spatially random crater distribution. If an area shows a clustered crater population, it either contains secondary craters or is not a homogeneous geologic unit. In both cases, any derived surface ages would likely be flawed. Requirements on defining a counting area are given in e.g. Neukum et al. (1975), Neukum and Hiller (1981), Neukum (1984), Wilhelms et al. (1987) and Michael and Neukum (2010). In order to identify clustering of craters, we perform randomness analysis on our measurements. This analysis of the spatial distribution of craters is a test in order to determine whether the measured spatial crater distribution is consistent with being accumulated on a homogeneous geological unit. We used 300 Monte Carlo iterations using the same area geometry as was used for the crater counting to find the degree of ordering or clustering of the measured craters relative to random distributions (Michael et al., 2012).

\subsection{Derivation of the crater production functions of asteroids}

The crater production function of asteroidal bodies and especially the body size-frequency distribution of asteroids has been discussed in many publications (e.g. Neukum et al., 1975; Neukum and Ivanov, 1994; Chapman et al., 1996a; Chapman et al., 1996b; Ivanov, 2001; Ivanov et al., 2001; Neukum et al., 2001; Werner et al., 2002; Bottke et al., 2005a; O'Brien and Greenberg, 2005; Strom et al., 2005; Tedesco et al., 2005; de Elía and Brunini, 2007; Ivanov, 2008; Gladman et al., 2009; Marchi et al., 2009; Head et al., 2010; Schmedemann et al., 2012). This matter is heavily debated for a number of reasons. Although asteroids are much more accessible to observations than any other source of potential projectiles such as Kuiper Belt Objects or comets and yet the asteroidal body distribution in the Main Belt is incompletely observed below about $5 \mathrm{~km}$ body diameter. De-biased censuses reach down to $\sim 1 \mathrm{~km}$ but incorporate a model assumption of the bias. Furthermore, there seems to be a discrepancy between the modeled body distribution and the observed crater distribution at small diameters $(\varnothing<5 \mathrm{~km}$ ). Models (Dohnanyi, 1971; Bottke et al., 2005a; Gladman et al., 2009) predict a shallower distribution than is observed on planetary surfaces. On larger bodies such as the Moon or Mars, secondary cratering has been suggested as being responsible for the observed steep distributions (e.g. Shoemaker, 1965). However, because of the low surface gravity, this explanation is not applicable to small asteroids such as Gaspra. Thus, it was concluded that the primary impactor population has a steep slope on the order of -3.5 cumulative (Neukum and Ivanov, 1994; Chapman et al., 1996b). However, it has been suggested that Gaspra's steep crater distribution may be the result of a short intense bombardment by Babtistina family fragments (Bottke et al., 2007). Other asteroids, such as Ida (Chapman et al., 1996a) and Lutetia (Marchi et al., 2012b) also show similar steep crater size distributions (see also SOM Section 1), implying a similar bombardment by other asteroid collision families and, probably, a generally steep small crater size-frequency distribution.

Framework for our lunar-like production function for asteroids:

There is a dispute on whether the steep small crater distribution on various bodies is a result of secondary cratering (e.g. McEwen and Bierhaus, 2006). Ivanov (2006) reported that secondary craters do not contribute significantly to the small crater population on the lunar surface. The same is suggested by Richardson (2009). Thus, the steep lunar crater distribution is predominantly caused by primary projectiles of near-Earth objects (NEO; mainly escaped Main Belt asteroids). A similar conclusion with respect to the secondary crater contribution on Mars was reported by Werner et al. (2009) and Neukum et al. (2010). Furthermore, Werner et al. (2002) find a high correlation between the lunar crater size-frequency distribution and the NEO body size-frequency distribution. This finding is confirmed by Ivanov et al. (2002) with further conclusions:

i) The surfaces of all terrestrial planets were bombarded by a NEO-like collisionally evolved projectile population, whose projectile size-frequency distribution is also similar to the body size-frequency distribution in the asteroid Main Belt. As detailed later in this section NEOs derive predominantly from the Main Belt from which they escaped by action of radiation and gravimetric forces. Their lifetime on planet 
crossing orbits is relatively short. Thus, it may be assumed that the size distribution does not change very much in NEO due to mutual collisions and is mostly governed by the initial asteroid size distribution inside the Main Belt and the size dependent radiation forces. Gravimetric forces are independent of body sizes.

ii) Cometary impacts are relatively insignificant $(<\sim 10 \%$ of all impacts) on terrestrial bodies.

iii) Due to the common main-impactor source, the wellinvestigated lunar cratering record can be scaled to other bodies, where the same crater distribution is observed. This is described by Ivanov (2001).

iv) The projectile size-frequency distribution has been stable since $\sim 4 \mathrm{Ga}$ for projectiles forming lunar craters $<300 \mathrm{~km}$ (projectile size: $\sim 27 \mathrm{~km}$; Werner et al., 2002).

Although these points are not universally accepted in the literature, we use them for our theoretical approach. As mentioned, Neukum and Ivanov (1994), Chapman et al. (1996a) and Chapman et al.(1996b) conclude that the small crater size-frequency distribution on Gaspra and Ida has a lunar-like slope, which is steeper than modeled body size-frequency distributions of projectiles in the asteroid Main Belt (Dohnanyi, 1971; Bottke et al., 2005a; Gladman et al., 2009). Furthermore, for the crater distribution on Lutetia, we also find a good agreement with a lunar-like crater production function for slightly larger craters than observed on Gaspra and Ida with a wavy characteristic (SOM Section 1).

The discrepancy between modeled projectile distribution and observed crater distributions may be resolved by consideration of the altered projectile dynamics due to the Yarkovsky and YORP effects (e. g. Farinella et al., 1998; Chesley et al., 2003; O'Brien and Greenberg, 2005; Bottke et al., 2006; Lowry et al., 2007). The Yarkovsky effect is probably more relevant and causes a shift of the semi-major axis of a body due to the vector difference between absorbed and re-emitted radiation. The magnitude of the effect depends on several factors such as speed and direction of rotation and tilt of the rotation axis (all three governed by YORP effect) and thermal surface properties. The most important factor, however, is the mass and thus, the size of the body. Small bodies are much more affected than large bodies due to the smaller volume-to-surface ratio. O'Brien and Greenberg (2005) presents a diagram of the removal efficiency of bodies from the Main Belt with respect to body sizes due to the action of resonances and the Yarkovsky effect. The complexity of the influence of the Yarkovsky and YORP effects on the small asteroid size distribution is described by Morbidelli and Vokrouhlický (2003) and Bottke et al. (2006). Based on those publications a qualitative model for a lunarlike small crater distribution on Main Belt asteroids could be envisioned.

Mean motion resonances are a sink for all bodies in the Main Belt, because they depend on gravitational influences only. The higher removal efficiency of smaller bodies together with the inferred stability of the observed crater size-frequency distribution in the inner Solar System (Ivanov et al., 2002) requires a specific refresh rate of small bodies by fragmentation of larger bodies. Many of these fragments move from their orbit of liberation to any one resonance inside the Main Belt more or less in a random walk fashion due to subsequent collisions and the Yarkovsky and YORP effects. Inside the stronger resonances, the bodies become dynamically excited in a way that they are quickly thrown onto planet crossing trajectories (e.g. Ivanov, 2001; O'Brien and Greenberg, 2005). Thus, we see a transient population of small bodies in the asteroid Main Belt. Since these bodies are continuously replenished and because they continuously change their orbital characteristics due to subsequent collisions, the Yarkovsky and YORP effects, as well as weak secular resonances, these bodies will eventually cross the orbits of other asteroids as well before they enter a mean motion resonance and become planet crossing asteroids. Assuming that, over timescales of several tens of millions of years, the small projectiles are roughly equally distributed in the Main Belt outside the resonance zones, we expect that a constant portion of small projectiles end up either inside a resonance or collide with another asteroid. The flux of projectiles into resonance zones and onto the surfaces of other asteroids would be proportional to the absolute number of bodies in the Main Belt as proposed by Neukum (1984). This implies a similar time dependence in projectile fluxes on the inner Solar System bodies and the Main Belt asteroids but at absolute higher rates inside the Main Belt. Since cratering is a cumulative process, the observed small crater size-frequency distribution has to be steeper than the observed transient population of small projectiles. Thus, the Yarkovsky effect could explain why both the flatter modeled body size-frequency distribution with immediately cleaned out small particles and the steeper observed crater size frequency distribution may not necessarily be in disagreement. This model also explains why the small primary crater distributions on the Moon and asteroids such as Gaspra are so similar to each other. However, this is a preliminary Gedanken experiment, which has to be improved and tested by modeling work and more observations. The interaction of subsequent collisions, radiation forces and mean motion resonances is extremely complex. Thus, no consensus on its influence on the NEO size frequency distribution has yet been reached in the literature.

For larger projectiles ( $\varnothing>\sim 5 \mathrm{~km}$ ), we have a nearly complete observation of the asteroid Main Belt. Thus, we are able to filter out objects on crossing orbits with a target asteroid and derive a projectile size-frequency distribution by statistical analysis of orbital elements of observed bodies. de Elía and Brunini (2007) report in their Table 3 that an object of the inner Main Belt ( $\sim 2 \mathrm{AU}-2.5 \mathrm{AU}$ ), of which Vesta is a member, is impacted by a $93 \% / 7 \%$ ratio from inside/outside the 5:2 resonance gap ( $\sim 2.823 \mathrm{AU})$. This is important, because the amplitude of the wave in the asteroid body size-frequency distribution becomes shallower going from the inner Main Belt outwards (e.g. Tedesco et al., 2005; de Elía and Brunini, 2007). Thus, an impactor population derived from inside the $5: 2$ resonance $(a<2.823 \mathrm{AU})$ has a different shape than the average Main Belt (Fig. 1).

de Elía and Brunini (2007) further report that $~ 94 \%$ of the asteroidal NEOs come from inside the 5:2 resonance. A similar result is also reported by Bottke et al. (2002). Thus, the projectile population hitting the Moon (NEOs) and Vesta (inner Main Belt asteroid) is nearly identical (Fig. 1). The above mentioned circumstances justify our decision to scale the lunar crater production function to vestan impact conditions, instead of scaling the average Main Belt size-frequency distribution to vestan crater sizes as has been done by Marchi et al. (2012c).

Our attempt to scale the lunar production function to vestan impact conditions has the advantage of being independent of models of unobserved populations of small projectiles. The lunar cratering record provides valid crater diameters at least down to about $200 \mathrm{~m}$ (van der Bogert et al., 2010), which corresponds to projectile diameters of a few tens of meters, which is far better than any direct observation of Main Belt asteroids will achieve within the near future. For the lunar case the widely used production function is defined for the diameter range of $10 \mathrm{~m}-300 \mathrm{~km}$ (Neukum, 1984).

The framework we use is possibly not in agreement with the E-belt model (Bottke et al., 2012). If the E-belt model is correct and the vast majority of the NEOs come from inside the $v_{6}$ resonance, we would expect larger amplitude in the wave in the $R$-plot of crater size-frequency distribution on the terrestrial planets than what is actually observed for the past $\sim 4 \mathrm{Ga}$ (based on Fig.1, De Elía and Brunini (2007) and Tedesco et al. (2005)). 
A

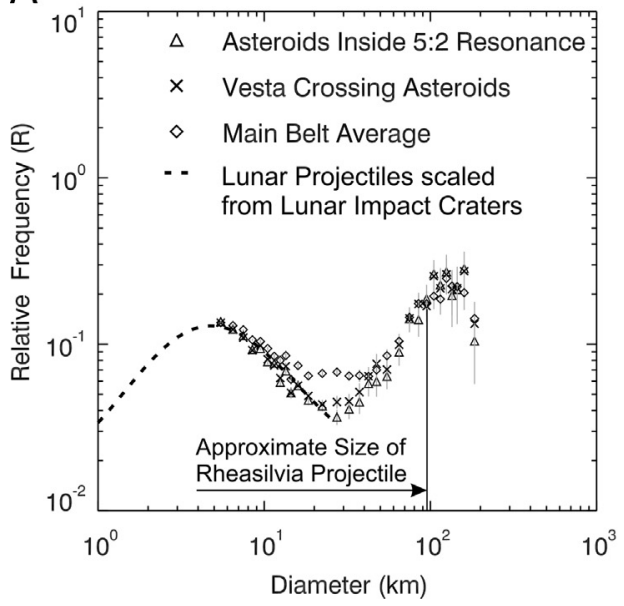

B

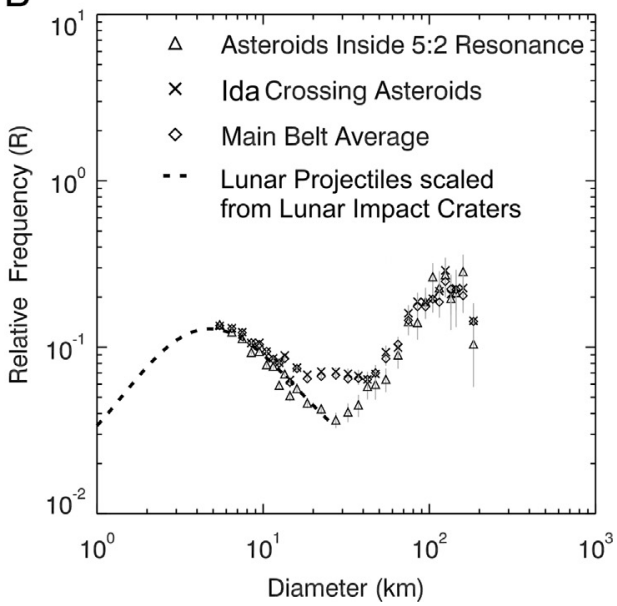

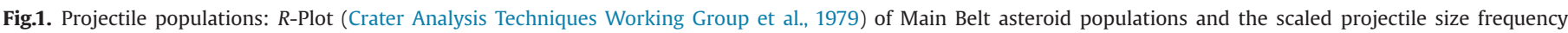

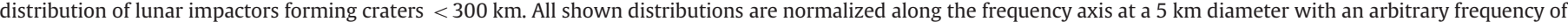

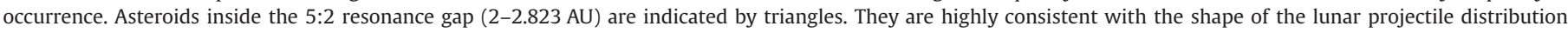

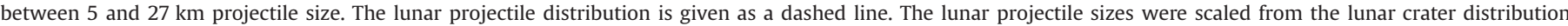

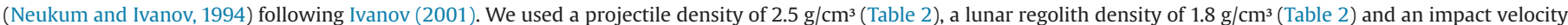

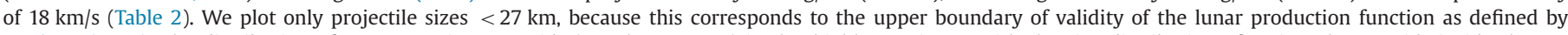

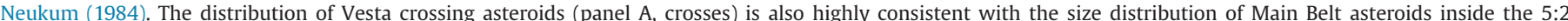

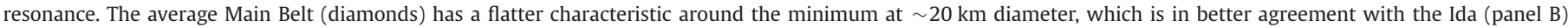

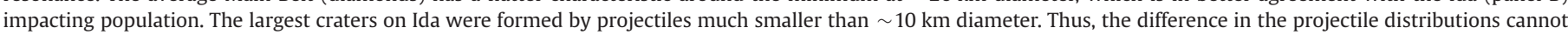

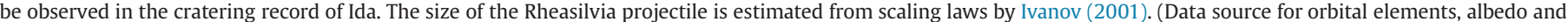
absolute magnitude: JPL Solar System Dynamics web page (http://ssd.jpl.nasa.gov/sbdb_query.cgi))

There is also an ongoing discussion of whether or not and when the lunar projectile distribution changed its shape. Several papers (e.g. Strom, 1977; Strom et al., 2005; Malhotra and Strom, 2011) discuss two different impactor populations on the terrestrial planets. This view is questioned by other authors (e.g. Neukum and Ivanov, 1994; Ćuk et al., 2010) for different reasons. Neukum and Ivanov (1994) suspect the source of the change between the pre- and post-Imbrian crater size-frequency distribution in resurfacing effects such as ejecta blanketing. Ćuk et al. (2010) questions the related dynamics of putative lunar cataclysm projectiles. There is also some divergence in the timing of the postulated population change (e.g. Head et al., 2010; Fassett et al., 2012; Marchi et al., 2012a). Thus, so far it is uncertain whether a change of the lunar projectile distribution occurred and how exactly it is characterized. For this work we assume a constant projectile size-frequency distribution over the last $4 \mathrm{Ga}$ as outlined above.

\subsubsection{Scaling of the lunar crater production function to asteroids}

In this work we use the corrected scaling laws by Ivanov (2001). We elected to use this kind of scaling because the related parameters can either be measured on the planetary surfaces or roughly estimated from well-known parameters such as the surface gravity. Another choice could have been the $\pi$-group scaling (Housen and Holsapple, 2011). In that case, important coefficients for strength and cohesion are only known for analog materials. Eq. (1) gives the corrected version of the Ivanov (2001) scaling.

$\frac{D_{t}}{D_{P}(\delta / \rho)^{0.43}(v \sin \alpha)^{0.55}}=\frac{1.21}{\left[\left(D_{s g}+D_{t}\right) g\right]^{0.28}}$

After Ivanov (2001; corrected by Ivanov and Hartmann (2007) and by Ivanov (2008) as his Eq. 8)

Table 2 summarizes the used scaling parameters. In Fig. 2, we show the distribution of impact probabilities versus impact velocities for the asteroids Vesta, Lutetia, Ida and Gaspra. For the orbital elements of the target bodies we use proper elements of the AstDys catalog as of July 212012 (http://hamilton.dm. unipi.it/astdys/index.php?pc=5). Orbital elements of the crossing field bodies are taken from the JPL Solar System Dynamics database as of July 212012 (http://ssd.jpl.nasa.gov/ sbdb_query.cgi). For this analysis, we used a diameter cut-off at $5 \mathrm{~km}$ body size. Above this diameter, we expect a nearly complete observation of bodies in the Main Belt between 2.1 and $4.1 \mathrm{AU}$. We estimated the body diameters by converting absolute magnitude and albedo values into absolute body diameters (Tedesco et al., 1992). For this purpose we derived a floating average of known albedo values with a box size of 0.5 AU. For all bodies with unknown albedo we used the calculated floating average for that particular region of the Main Belt in order to derive asteroid diameters. The impact probabilities of the four mentioned asteroids are used for the derivation of their chronologies (next section). Here we use the average impact velocities to scale the lunar crater production function to the impact conditions at the four mentioned target asteroids. The impact probability-impact velocity plot shows distinct shoulders of higher impact probability between 6 and $8 \mathrm{~km} / \mathrm{s}$ for Vesta and Ida (Ida: 6-7 km/s). If the responsible bodies are plotted with inclination vs. semi-major axis, we see basically three outstanding clusters for Vesta and two for Ida. The clustering in the regions of the Eunomia and Hansa family suggest that some family members could indeed be responsible for higher impact probabilities in the impact velocity range of 6$8 \mathrm{~km} / \mathrm{s}$. The third cluster is in the area of the Theobalda family. This family is not as densely populated as the Eunomia family, but many of the Vesta and Ida crossing bodies are located in this region. An actual connection to the Theobalda family is therefore questionable. Solving this matter is beyond the scope of this work. For now we conclude that the observed shoulders in the probability-velocity plot can be linked to certain body populations in the Main Belt. If in such populations a break up event occurs, it might increase the rate of bombardment at the target asteroid and might also have an influence on the impactor-size spectrum for a certain amount of time. The production and chronology functions we derive here are averaged over time. The actual function shapes and values for a specific point in time may diverge from this average. 
Table 2

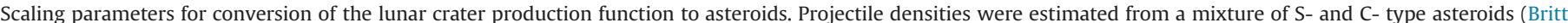

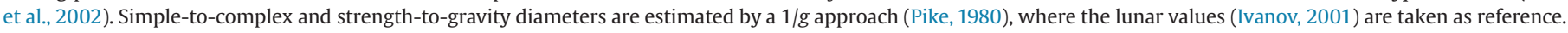

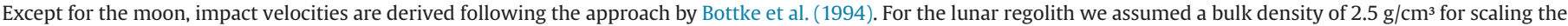

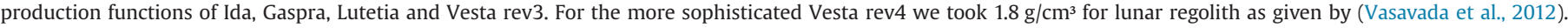

\begin{tabular}{|c|c|c|c|c|c|c|}
\hline Body & Target density $\left[\mathrm{g} / \mathrm{cm}^{3}\right]$ & $\begin{array}{l}\text { Projectile } \\
\text { density } \\
{\left[\mathrm{g} / \mathrm{cm}^{3}\right]}\end{array}$ & $\begin{array}{l}\text { Simple to complex transition } \\
{[\mathrm{km}]}\end{array}$ & $\begin{array}{l}\text { Strength to gravity transition } \\
{[\mathrm{km}]}\end{array}$ & Impact velocity $[\mathrm{km} / \mathrm{s}]$ & Surface gravity $\left[\mathrm{m} / \mathrm{s}^{2}\right]$ \\
\hline Moon & $\begin{array}{c}2.5 \\
\text { except for Vesta rev4 } \\
\text { Vesta rev4: } 1.8 \\
\text { Vasavada et al. (2012) }\end{array}$ & 2.5 & $\begin{array}{c}15 \\
\text { Ivanov (2001) }\end{array}$ & $\begin{array}{c}0.3 \\
\text { Ivanov }(2001)\end{array}$ & $\begin{array}{c}18 \\
\text { Ivanov (2008) }\end{array}$ & $\begin{array}{c}1.62 \\
\text { Ivanov (2008) }\end{array}$ \\
\hline Vesta & $\begin{array}{c}3 \\
\text { (basaltic regolith; } \\
\text { Russell et al., 2012) }\end{array}$ & 2.5 & $\begin{array}{l}70 \\
\text { (est. from the transitional } \\
\text { character of Marcia crater } \\
\text { on Vesta) }\end{array}$ & $\begin{array}{c}\text { rev3: } 2.21 \\
\text { (est. from pre-Dawn } \\
\text { mass of Vesta; } \\
\text { (Thomas et al., 1997)) } \\
\text { rev4: } 1.94 \\
\text { (est. from Russell et al., 2012) }\end{array}$ & $\begin{array}{c}\text { rev3: } 4.75 \\
\text { (pers. comm. O'Brien) } \\
\text { rev4: } 4.56 \\
\text { (est. after Bottke et al., 1994) }\end{array}$ & $\begin{array}{c}\text { rev3: } 0.22 \\
\text { (est. from pre- Dawn mass of } \\
\text { Vesta; (Thomas et al., 1997)) } \\
\text { rev4: } 0.25 \\
\text { Russell et al., } 2012\end{array}$ \\
\hline Lutetia & $\begin{array}{c}3.3 \\
\text { Thomas et al. (2012) }\end{array}$ & 2.5 & $\begin{array}{c}2430 \\
\text { (est. from Pätzold et al., 2011) }\end{array}$ & $\begin{array}{c}49 \\
\text { (est. from Pätzold et al., 2011) }\end{array}$ & $\begin{array}{c}4.09 \\
\text { (est. after Bottke et al., 1994) }\end{array}$ & $\begin{array}{c}0.01 \\
\text { Pätzold et al. (2011) }\end{array}$ \\
\hline Ida & $\begin{array}{c}2.6 \\
\text { Belton et al. (1996) }\end{array}$ & 2.5 & $\begin{array}{c}3471 \\
\text { (est. from Thomas et al., 1996) }\end{array}$ & $\begin{array}{c}69 \\
\text { (est. from Thomas et al., 1996) }\end{array}$ & $\begin{array}{c}3.32 \\
\text { (est. after Bottke et al., 1994) }\end{array}$ & $\begin{array}{c}0.007 \\
\text { Thomas et al. (1996) }\end{array}$ \\
\hline Gaspra & $\begin{array}{c}2.7 \\
\text { Britt et al. (2002) }\end{array}$ & 2.5 & $\begin{array}{l}4860 \\
\text { (est. from Thomas et al., 1994) }\end{array}$ & $\begin{array}{c}97 \\
\text { (est. from } \\
\text { Thomas et al., 1994) }\end{array}$ & $\begin{array}{c}4.69 \\
\text { (est. after Bottke et al., 1994) }\end{array}$ & $\begin{array}{l}0.005 \\
\text { Thomas et al. (1994) }\end{array}$ \\
\hline
\end{tabular}

A

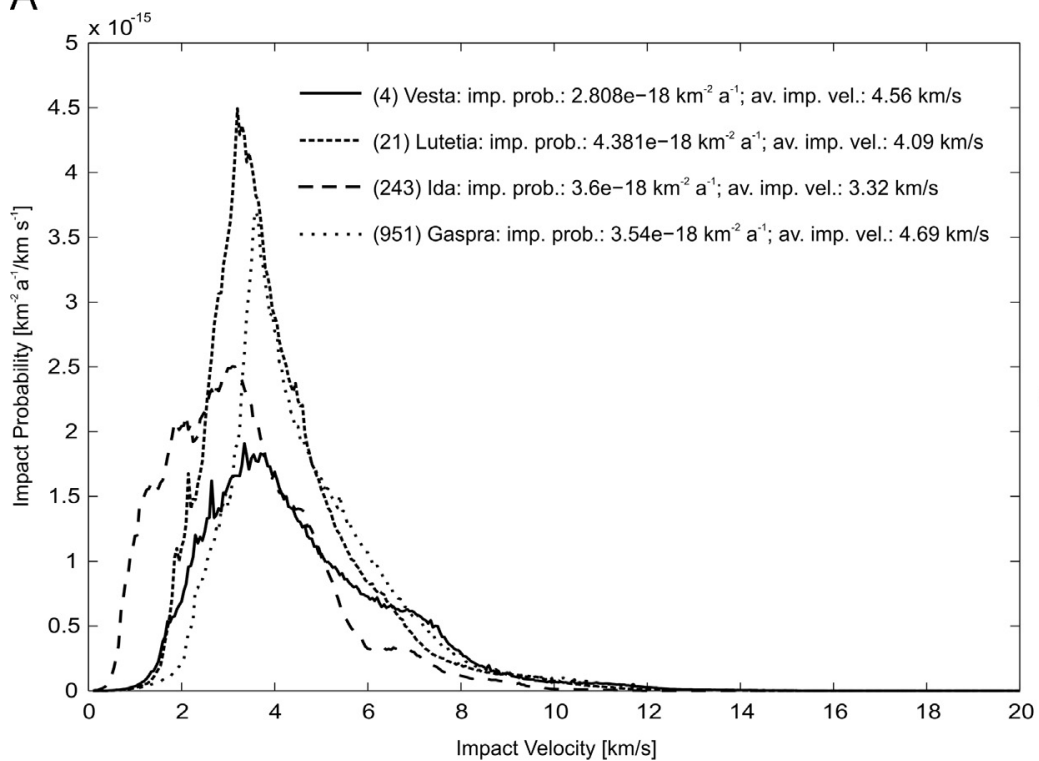

B

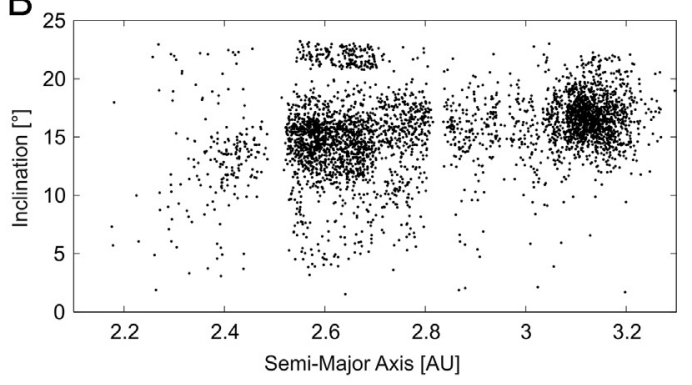

$\mathrm{C}_{25}$

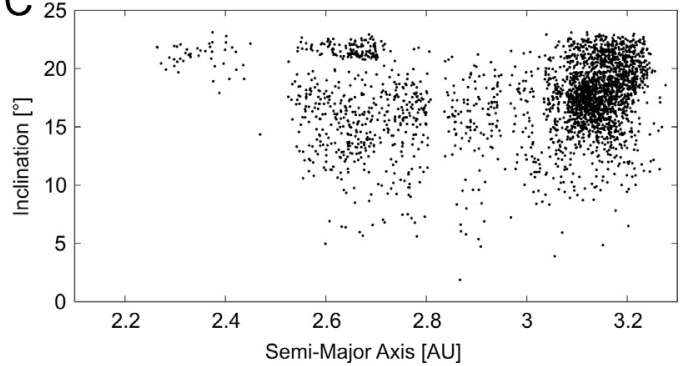

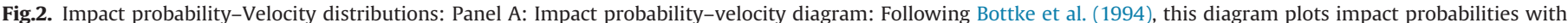

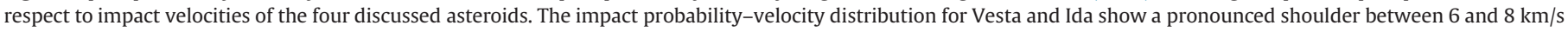

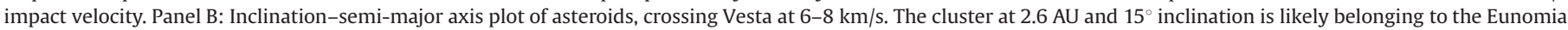

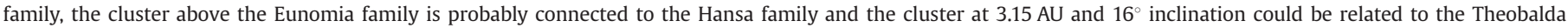

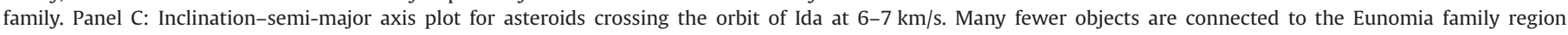

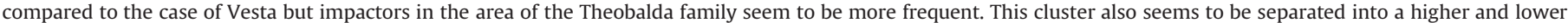
inclination part.

Eq. (2) defines the polynomial of 11th degree for crater production functions (Neukum, 1984; Neukum and Ivanov, 1994; Neukum et al., 2001). In this equation, $N_{\text {cum }}$ is the cumulative crater frequency for craters equal or larger than the crater diameter $D$ and $a_{x}$ are the coefficients of the individual terms.

$\log N_{\text {cum }}=a_{0}+a_{1} \log (D)+a_{2}(\log (D))^{2}+\ldots+a_{11}(\log (D))^{11}$

Table 3 provides the respective values for the used coefficients for the Moon, which crater production function has been used as reference, for Vesta (rev3+4), Lutetia, Ida and Gaspra. Here we give two revisions for crater production functions of Vesta which are quite similar in shape. However, the respective chronologies for these cases differ slightly from each other, and for data integrity we present crater production functions and chronologies of both revisions here. Vesta rev3 has been derived from pre- and early-Dawn data and has been used in several publications already. Rev4 on the other hand, incorporates the most recent results of the Dawn mission and a revised bulk density of the lunar regolith (Vasavada et al., 2012).

Fig. 3 visualizes the derived crater production functions of the asteroids and the lunar reference function. 
Table 3

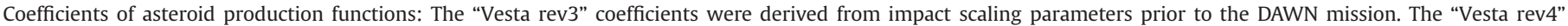
coefficients are an update by utilizing impact scaling parameters based on results of the DAWN mission and an updated bulk density of the lunar regolith.

\begin{tabular}{|c|c|c|c|c|c|c|}
\hline & Moon Neukum (1984) & Vesta rev3 & Vesta rev4 & Lutetia & Ida & Gaspra \\
\hline$a_{0}$ & -2.5339 & -3.1643 & -3.365 & -3.196963 & -3.224142 & -2.994946 \\
\hline$a_{1}$ & -3.6269 & -3.0382 & -2.9267 & -2.750391 & -2.734469 & -2.837653 \\
\hline$a_{2}$ & 0.43662 & 0.5445 & 0.6192 & 0.655547 & 0.671929 & 0.58864 \\
\hline$a_{3}$ & 0.79347 & 0.67305 & 0.59636 & 0.320959 & 0.324531 & 0.362687 \\
\hline$a_{4}$ & 0.086468 & 0.11447 & 0.11879 & -0.101901 & -0.117051 & -0.090568 \\
\hline$a_{5}$ & -0.26485 & -0.34186 & -0.30577 & -0.051678 & -0.061444 & -0.081317 \\
\hline$a_{6}$ & -0.066382 & -0.15077 & -0.20515 & 0.016547 & 0.025122 & 0.033631 \\
\hline$a_{7}$ & 0.037923 & 0.079115 & 0.068576 & -0.020969 & -0.016168 & -0.00511 \\
\hline$a_{8}$ & 0.010596 & 0.035557 & 0.058513 & -0.008409 & -0.010554 & -0.015223 \\
\hline$a_{9}$ & -0.0022496 & -0.0099727 & -0.010293 & 0.007004 & 0.006315 & 0.004495 \\
\hline$a_{10}$ & -0.00051797 & -0.002574 & -0.0053325 & 0.001212 & 0.001419 & 0.00205 \\
\hline$a_{11}$ & 0.0000397 & 0.00058434 & 0.0009643 & -0.000638 & -0.000625 & -0.000613 \\
\hline
\end{tabular}

\subsection{Discussion of alternative proposed chronology model}

In the previous sections we stated our reasoning for believing that the Moon and Asteroids are cratered by impactor populations with similar size-frequency distributions. In our model we also expect the impact rate on the terrestrial planets and the Main Belt asteroids to be characterized by a similar time-dependent evolution (Neukum et al., 2001; Ivanov et al., 2002). Imaging data of freshly formed craters has allowed for direct measurement of the present day impact flux on Mars, revealing a high degree of correspondence between the lunar and the scaled lunar-like Martian production function and chronology (Malin et al., 2006; Kreslavsky, 2007; Daubar et al., 2013). This result further supports the idea of a common projectile population at least for the Moon and Mars and demonstrated the consistency of current impact rates derived from statistical analysis of asteroid orbits.

Morbidelli et al. (2012) report a good agreement with the lunar chronology by Neukum and Ivanov (1994) over the last 4.1 Ga, based on the Nice model (e.g. Gomes et al., 2005; Morbidelli et al., 2005; Tsiganis et al., 2005) and the E-Belt model (Bottke et al., 2012). The Nice model is a numerical computer simulation in which, due to a resonance crossing between Jupiter and Saturn, the small body populations in the Solar System are destabilized and dynamically excited. Furthermore, in some flavors of the Nice model, Uranus and Neptune switch their order. In general, the outer planet orbits change from a more compact configuration to the currently observed one. The shape of a Nice- and E-Belt model based chronology function of a target body should be different depending on whether the target object is inside ( $a_{\text {target }}<a_{\text {source }}$; terrestrial planets) or outside ( $a_{\text {target }}>a_{\text {source }}$; Main Belt asteroids) the semi-major axis range of the E-Belt. The Hungaria asteroid family is suggested to be the remains of an initial much heavier E-Belt (up to 0.8 times the current Main Belt) located between $\sim 1.7$ and 2.1 AU. According to the E-Belt model (Bottke et al., 2012) the E-Belt would contribute 10 times more asteroids to the inner planet region than the entire remaining asteroid Main Belt outside the $\mathrm{v}_{6}$ resonance although the initial mass of the E-Belt is at most $80 \%$ that of the current entire Main Belt. Thus, the E-Belt would be the prime source for NEOs during the proposed late heavy bombardment time period.

In the work of Morbidelli et al. (2012) the onset of the so-called late heavy bombardment (LHB) was shifted backwards in time by $200 \mathrm{Ma}$ compared to the original idea of a terminal lunar cataclysm around 3.9 Ga ago (Tera et al., 1974). Also the duration of the LHB period changed from a few tens of millions of years (Gomes et al., 2005) to roughly 400 Ma years for the end of the conventional LHB at around 3.7 to $3.8 \mathrm{Ga}$ (Bottke et al., 2012). The same work suggests that the LHB was even more extended by stating that probably one E-Belt projectile could have hit the Moon as late as 1.5-3.2 Ga ago. This suggestion is supported by crater measurements by Kirchoff et al. (2013) and a reevaluation of lunar chronology data (Le Feuvre and Wieczorek, 2011) but is in disagreement with Chapman et al. (2007), who argue for a cessation in lunar impact rates within $\sim 0.05-0.1 \mathrm{Ga}$ declining from high rates at $3.9 \mathrm{Ga}$. Furthermore, in the Nice model the projectile characteristic changed from roughly equal contributions by asteroids and short period comets with a slight time delay in the impact spike for the asteroids (Gomes et al., 2005) to a heavy preponderance of asteroids, predominantly E-Belt asteroids (Bottke et al., 2012).

Although the lunar cataclysm in the shape of the Nice model has been quite popular in recent years, the basic idea of a lunar cataclysm has been heavily debated (e.g. Baldwin, 1974; Hartmann, 1975, 2003; Neukum, 1984; Haskin et al., 1998; Baldwin, 2006; Nimmo and Korycansky, 2012; Fernandes et al., 2013) since it was proposed by Tera et al. (1974). The alternative for the lunar cataclysm model is some kind of a smooth decay in impact rate as it was suggested for the Moon and other inner Solar System bodies (e.g. Wetherill, 1975; Neukum, 1984; Hartmann and Neukum, 2001; Le Feuvre and Wieczorek, 2011). Interestingly since the paper by Morbidelli et al. (2012) there is a convergence at least for the Moon between the two camps (pro/contra lunar cataclysm), which agree on the lunar chronology over the last $4.1 \mathrm{Ga}$. O'Brien et al. (under review) presents a cratering chronology for Vesta based upon the latest developments in the Nice model. Due to Vesta's peculiar dynamical situation inside the Main Belt O'Brien's chronology model differs significantly from typical LHB chronology models of the inner Solar System bodies. O'Brien's chronology for Vesta agrees with ours from the present back to $\sim 3 \mathrm{Ga}$. At earlier times both chronologies diverge significantly in a way that the lunar-like chronology for Vesta turns over into an exponential part, which describes the smooth decay in impact rates for the time period of the declining heavy bombardment. The Nice model derived chronology for Vesta by O'Brien et al. shows only a minor increase in impact rates at $4.1 \mathrm{Ga}$ and a very steep increase close to $4.5 \mathrm{Ga}$. In the linear part, for ages $<\sim 3 \mathrm{Ga}$, this characteristics leads to similar results between both chronologies and nearly the same $\mathrm{N}(1)$ value (i.e. crater frequency at $1 \mathrm{~km}$ crater diameter). The Nice model chronology produces increasingly older ages at the same crater frequency where the lunar-like chronology steepens up in the exponential part for surface ages $>\sim 3 \mathrm{Ga}$. One fundamental assumption in the Nice model based chronology for Vesta by O'Brien et al. is that the surface of Vesta shows a complete cratering record above $200 \mathrm{~km}$ crater size since $4.56 \mathrm{Ga}$. In other words, the crater frequency at $200 \mathrm{~km}$ crater size (N200) is set to $4.56 \mathrm{Ga}$. At least for one $\sim 180 \mathrm{~km}$ crater 


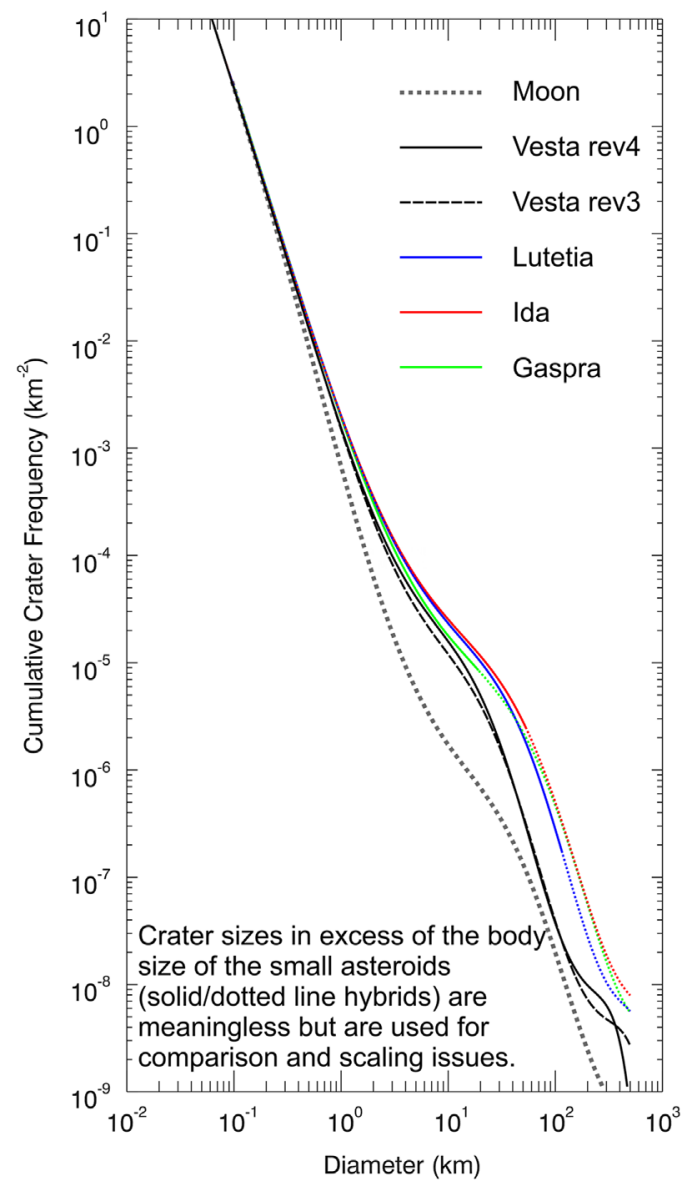

Fig. 3. Lunar and asteroidal crater production functions: All production functions are normalized in cumulative crater frequency at $100 \mathrm{~m}$ crater diameter and therefore do not represent the same model ages. The lunar production function is the steepest, due to the comparatively high surface gravity and high impact velocities. The two Vesta curves are very similar but diverge around $10 \mathrm{~km}$ and at very large diameters $(200-500 \mathrm{~km})$. The differences around $10 \mathrm{~km}$ are due to the change of the lunar bulk density from $2.5 \mathrm{~g} / \mathrm{cm}^{3}$ in rev3 to $1.8 \mathrm{~g} / \mathrm{cm}^{3}$ in rev4. Differences for the largest craters are likely due to an instability of the polynomial function in that range and an optimization of the curve fitting at small diameters. Thus, fits to measured crater frequencies at diameters $>100 \mathrm{~km}$ should be evaluated with caution. The Vesta production functions are transitional between the Moon and smaller asteroids because of the intermediate mass of Vesta. Although Gaspra is the smallest body between the small asteroids its production function is slightly steeper than the curves of Lutetia or Ida below $100 \mathrm{~km}$ diameter. This could be caused by the higher average impact velocity $(4.69 \mathrm{~km} / \mathrm{s})$ compared to Lutetia $(4.09 \mathrm{~km} / \mathrm{s})$ or Ida $(3.32 \mathrm{~km} / \mathrm{s})$. The curves of the small asteroids are calculated beyond the body size (dotted lines) for better comparison as well as for scaling of the asteroidal chronologies. In reality, craters larger than the body diameter will result in catastrophic disruption of the target body.

$\left(30^{\circ} \mathrm{N} / 250^{\circ} \mathrm{E}\right.$; Fig.11) there is morphologic evidence, that it suffered severe degradation at its northern rim presumably due to the tectonic response caused by the formation of large basins. Several other basins may not have been recognized yet because their morphology is heavily muted. For instance, the average height in the heavily cratered terrain $\left(\sim 10^{\circ}-40^{\circ} \mathrm{N} / \sim 30-150^{\circ} \mathrm{E}\right)$ is so low that the crater floors of even small craters $(\varnothing \sim 20 \mathrm{~km})$ reach $\sim 20 \mathrm{~km}$ below the reference ellipsoid. The assumption for the Nice model based chronology implies that Vesta has the oldest surface in the Solar System, as far as has been investigated. It would be even older than the densely cratered surface of Iapetus, whose surface age is estimated to be $\sim 4.4 \mathrm{Ga}$ (Castillo-Rogez et al., 2007). The cumulative crater frequency at Iapetus (Zahnle et al., 2003; Neukum et al., 2006) for $4 \mathrm{Ga}$ of model age can roughly be estimated at least 2 orders of magnitude less than the crater frequency at Vesta (current cratering rate at the Moon $\sim 24$ times less than at Vesta; see Table 4 and Fig. 3). Therefore, it appears somewhat unlikely that all basins ever formed on Vesta are still detectable today. For comparison, in the lunar-like chronology the $N(100)$ value (frequency of craters $\geq 100 \mathrm{~km}$ diameter) for the total surface area of Vesta gives a surface model age between 3.9 and $4 \mathrm{Ga}$ (SOM, Section 3). Since the lunar chronology is still debated for ages $>\sim 4.1 \mathrm{Ga}$ and radioisotopic $\mathrm{Ar}^{39}-\mathrm{Ar}^{40}$ ages of Vesta derived brecciated HED meteorites lack ages $>\sim 4.1$ Ga (Bogard, 2011) we make no assumptions about the vestan chronology for ages $>4.1 \mathrm{Ga}$, because it appears not to be accessible to crater measurements in the frame of lunar derived functions. Although the chronology by O'Brien et al. has been developed for the case of Vesta from the most recent dynamical models, we question the approach for the mentioned reasons. However, this is the only alternative chronology for Vesta available in order to estimate crater retention ages. Thus, we use it (hereafter "O'Brien's chronology") to compare its predictions with our lunar-like approach.

\subsubsection{Lunar-like chronology of asteroids}

Based on assumptions we made in the previous sections Eq. (3) defines the lunar-like chronology function following Neukum (1984), Neukum and Ivanov (1994), Neukum et al. (2001) and Marchi et al. (2009). In this equation $N_{\text {cum }}$ is the cumulative crater frequency for craters $\geq 1 \mathrm{~km}, k_{1-3}$ are the used coefficients and $t$ is the surface age in Ga.

$N_{\text {cum }}(D \geq 1 \mathrm{~km})=k_{1}\left(e^{k_{2} t}-1\right)+k_{3} t$

$k_{3}$ resembles the linear term in Eq. (3), which is equivalent to the current rate of collisions. Using Eq. (4), we can calculate the number of collisions per Ga per $\mathrm{km}^{2}$ from the observed orbital parameters of the target body and crossing Main Belt asteroids. $P_{i}$ is the intrinsic collision probability (Bottke et al., 1994), $r_{t}$ and $r_{p}$ are the radii of the target body and the projectile respectively. Since the average projectile size is much smaller than the target body, we assume $\left(r_{t}+r_{p}\right)^{2} \approx r_{t}$ and cancels out the $r_{t}^{2}$ term. $N$ is the number of available projectiles.

$k_{3}=\frac{P_{i}\left(r_{t}+r_{p}\right)^{2} N * 10^{9}}{4 \pi r_{t}^{2}}$

as given by O'Brien and Greenberg (2005).

Eq. (3) calculates the cumulative number of craters $\geq 1 \mathrm{~km}$. Therefore in Eq. (4), " $N$ " would be the number of projectiles forming craters $\geq 1 \mathrm{~km}$. Projectiles forming $1 \mathrm{~km}$ craters are only $\sim 65 \mathrm{~m}$ in diameter. Thus, the number of the projectiles cannot be determined from observations of the asteroid Main Belt alone, because the observational completeness is at about $5 \mathrm{~km}$ body size, although de-biased censuses reach $\sim 1 \mathrm{~km}$. We use the number of Main Belt asteroids $\geq 10 \mathrm{~km}$ and infer from the scaling laws the expected crater diameter at the surfaces of the target asteroids. For example on Vesta, a $10 \mathrm{~km}$ projectile is expected to form a $63 \mathrm{~km}$ crater. From the crater production function we calculate the ratio of the formation rate of craters $\geq 1 \mathrm{~km}$ to that of craters $\geq 63 \mathrm{~km}$ and multiply this ratio with the number of projectiles $\geq 10 \mathrm{~km}$.

For $N$ we find 9404 objects in the JPL SSD catalog between 1.8 and $4.1 \mathrm{AU}$ with diameters $\geq 10 \mathrm{~km}$. The Vesta crater production function gives a ratio of 9262 . Thus, we find $N=9404 \times$ $9262=87.1 \times 10^{6}$.

The $k_{2}$ value is the decay constant of the exponential decay. $k_{1}$ weights the exponential term and is scaled to the lunar value with the ratio of $k_{3 \text {-asteroid }} / k_{3 \text {-lunar }}$.

In Table 4 we give the respective values of the derived coefficients of the asteroid chronology functions. The lunar coefficients are also given as reference. 
Table 4

Intrinsic collision probabilities ( $P_{i}$; Fig.2) for asteroids and coefficients $k_{1-3}$ of lunar and asteroidal chronology functions.

\begin{tabular}{lrrrl}
\hline & \multicolumn{1}{l}{$P_{i}$} & \multicolumn{1}{c}{$k_{1}$} & \multicolumn{1}{l}{$k_{2}$} & \multicolumn{1}{l}{$k_{3}$} \\
\hline Moon (Neukum, 1984) & & $5.44 \times 10^{-14}$ & 6.93 & $8.38 \times 10^{-4}$ \\
Vesta (rev3) & $2.717 \times 10^{-18}$ & $1.3223 \times 10^{-12}$ & 6.93 & 0.02037 \\
Vesta (rev4) & $2.808 \times 10^{-18}$ & $1.2850 \times 10^{-12}$ & 6.93 & 0.01979 \\
Lutetia & $4.381 \times 10^{-18}$ & $2.0479 \times 10^{-12}$ & 6.93 & 0.0315 \\
Ida & $3.6 \times 10^{-18}$ & $1.581 \times 10^{-12}$ & 6.93 & 0.0244 \\
Gaspra & $3.54 \times 10^{-18}$ & $2.6354 \times 10^{-12}$ & 6.93 & 0.0406 \\
\hline
\end{tabular}

Fig. 4 shows a semi-log plot of the expected cumulative crater frequencies of craters $\geq 1 \mathrm{~km}$ with respect to the surface age of the asteroids and the Moon.

\section{Results: crater retention ages for key areas}

In this section we interpret many of the kinks in the measured crater distributions as resurfacing events and not as the original production function. Especially on Vesta we find examples where we see such kinks in one area but not in another with similar age and similar diameter range such as the northern and southern ejecta blanket of the Oppia crater in Section 3.2.8 (additional kink in the southern area). Since crater degradation more efficiently removes small craters than large ones and due to the high impact rates on Vesta, resurfacing induced by seismic shaking and/or ejecta blanketing appears more reasonable than frequent changes in the projectile size-frequency distribution over wide time and diameter ranges. Thus, we think the frequently observed kinks in the crater distribution with shallower characteristics towards smaller sizes are less likely a result of a changing impactor population than the result of resurfacing effects. However, we accept that major break up events in the Main Belt and/or basin forming events on Vesta itself could produce enough secondary projectiles for a limited period of time to change the size distribution of the impacting projectiles to a steeper configuration. This could be the case for a large fraction of the northern hemisphere of Vesta, which appears to have been heavily contaminated with secondary craters from the Rheasilvia impact with probable contributions from the then, replenished population of Vestoids. Such large contributions of secondary projectiles in addition to the primary projectiles should always lead to a steeper crater distribution in a specific size range.

\subsection{Surface ages of Ida, Gaspra and Lutetia}

In this section we present results of measurements for key areas, which were used to test the derived production function for consistency with the observed and measured crater distribution. For these areas we also calculate surface ages based on the presented crater counts and derived crater production and chronology functions. Measurements from Ida, Gaspra and Lutetia are detailed in the SOM Section 1. In Table 5 we present our results from these smaller asteroids.

In general our results are in agreement with earlier works on these smaller asteroids (Chapman et al., 1996a, 1996b; Marchi et al., 2012b). The main differences between the earlier work and our attempt are the utilization of ArcGIS for crater counting and the size correction of measurements to account for the projection error caused by the divergence between the reference body and the actual topography. Chronology functions of the earlier work are based on the current intrinsic impact probability of the investigated target bodies, similar to our approach. Thus, similar results are not surprising as long as derived model ages fall into the linear part of our lunar-like chronologies.

A few heavily degraded craters on Gaspra give a loosely constrained age of $2.9_{-1.1}^{+0.4} \mathrm{Ga}$. Fresh craters indicate a much younger surface of $270 \pm 70 \mathrm{Ma}$. At that time Gaspra may have

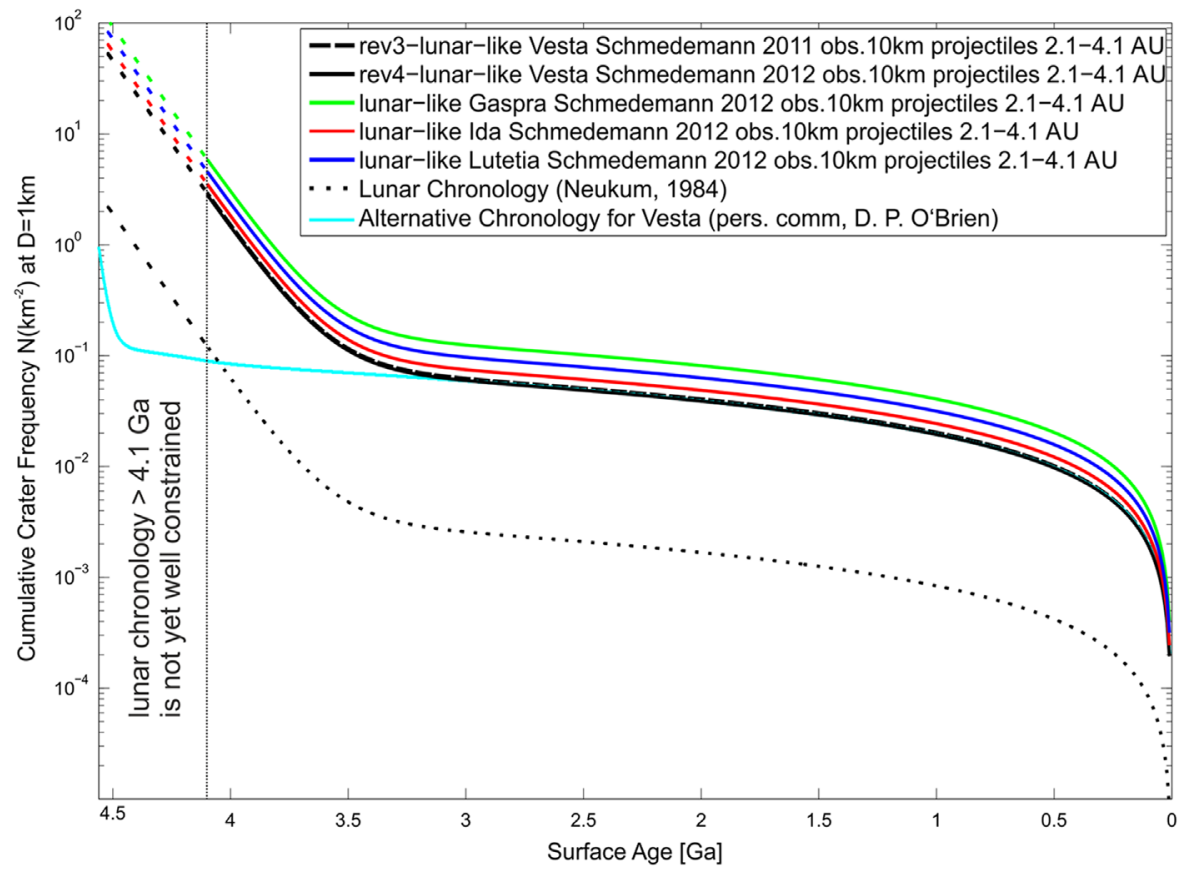

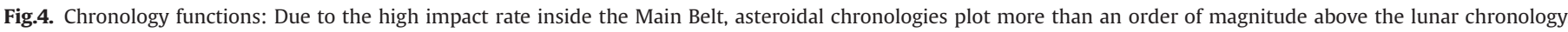

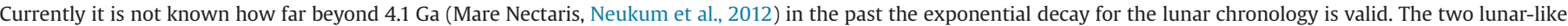

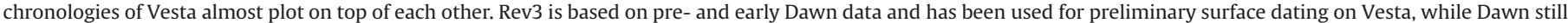

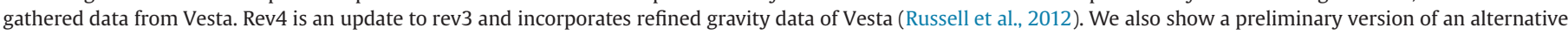

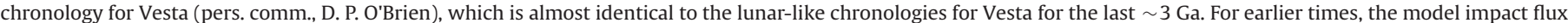
of O'Brien's chronology is much lower than that of our lunar-like chronology model for Vesta, so the same measured frequency produces older model ages. 
Table 5

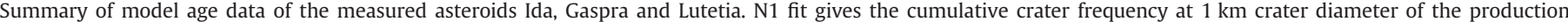

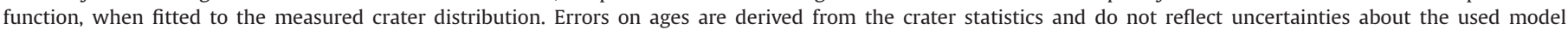
chronology. Maps and plots of each measurement are presented in the SOM (Section 1).

\begin{tabular}{|c|c|c|c|c|c|c|}
\hline Measurement & Lunar-like age [Ga] & Lunar-like age+Error [Ga] & Lunar-like age-Error [Ga] & N1 fit & $\mathrm{N} 1+$ Error fit & N1 - Error fit \\
\hline Gaspra_all_craters & 2.9 & 0.43 & 1.1 & 0.119 & 0.0446 & 0.0446 \\
\hline Gaspra_fresh_craters & 0.265 & 0.068 & 0.068 & 0.0107 & 0.00273 & 0.00273 \\
\hline Ida_all_craters & 3.59 & 0.058 & 0.097 & 0.188 & 0.0516 & 0.0516 \\
\hline Ida_fresh_craters Baseage & 3.35 & 0.18 & 1.3 & 0.1 & 0.0497 & 0.0497 \\
\hline Ida_fresh_craters Resurfacing age & 2.13 & 0.27 & 0.27 & 0.052 & 0.00664 & 0.00664 \\
\hline Lutetia_all_craters & 3.49 & 0.036 & 0.047 & 0.177 & 0.0201 & 0.0201 \\
\hline Lutetia_fresh_craters Resurfacing age & 2.08 & 0.25 & 0.25 & 0.0655 & 0.00804 & 0.00804 \\
\hline
\end{tabular}

collided with another asteroid, which caused widespread resurfacing.

Fresh craters on Ida are probably in saturation below $\sim 1 \mathrm{~km}$ crater size. Thus, the derived age may only be a lower limit. Similarly to the earlier work by Chapman et al. (1996a), we find a model age of $2.1 \pm 0.3 \mathrm{Ga}$. Craters $\geq 1 \mathrm{~km}$ give a surface age of $3.4_{-1.3}^{+0.2} \mathrm{Ga}$ and if heavily degraded craters are also taken into account we find an age of $3.6 \pm 0.1 \mathrm{Ga}$ for Ida.

Interestingly, we find a similar age using Lutetia's large and degraded craters $(3.5 \pm 0.1 \mathrm{Ga})$. This is about 200 Ma younger than the result by Marchi et al. (2012b). Fresh craters $<7 \mathrm{~km}$ on Lutetia indicate a surface model age of $2.1 \pm 0.3 \mathrm{Ga}$. The most important difference between our work and that of Marchi et al. (2012b) is that we interpret the lower crater frequency of fresh craters $<7 \mathrm{~km}$ as a younger crater population. That population built up after a resurfacing event such as the formation of a large impact crater, which may have caused severe seismic shaking and some degree of ejecta blanketing, despite the low surface gravity. Preexisting craters were degraded but not completely destroyed. Their morphology however, was muted to a point that Marchi et al. (2012b) did not accept them as craters anymore. Instead, they interpreted the kink in their crater distribution as a result of different scaling between two layers of more and less competent materials.

\subsection{Surface Ages of Vesta}

For Vesta we present surface model ages for both models: our lunar-like model and that based on the Nice model (O'Brien et al., under review). We will refer to the Nice model based chronology also as O'Brien's chronology. Both model ages are derived from the same $N(1)$ value, which is calculated from the fit of our lunar-like production function for Vesta to the measured crater distribution.

The vestan cratering record appears to be affected by an additional distinct population of impactors in a limited size range. This effect may either be linked to the influence of the Vesta collisional family, whose members are also known as Vestoids (e.g. Nesvorný et al., 2008), or to secondary cratering, both caused by the formation of large basins such as the Rheasilvia South Pole basin (e.g. Jaumann et al., 2012). The additional projectile population appears to influence the vestan cratering record only in a limited diameter range of about $7-12 \mathrm{~km}$ crater size and only in the northern hemisphere of Vesta (i.e. outside the Rheasilvia basin) to variable amounts. A contamination of the smaller crater distribution $(<\sim 7 \mathrm{~km})$ with secondary craters might be possible, but only to a minor degree such that it does not dominate the crater distribution. Alternatively, the crater distribution in that size range could also be heavily influenced by secondary cratering, which is not indicated by a steep crater distribution. It might be possible that due to high crater frequencies saturation effects occur, that prevent the observation of a steep crater distribution.
The presented Dawn imaging data of Vesta is projected to a $255 \mathrm{~km}$ radius sphere. Surface areas as well as crater diameters were measured on that sphere and corrected for the projection error caused by divergences between the reference body and the actual topography (Kneissl et al., 2014). The topographic information is related to a vestan ellipsoid with a semi major axis of $285 \mathrm{~km}$ and a semi-minor axis of $229 \mathrm{~km}$ (Jaumann et al., 2012). For large counting areas, we used the Dawn high altitude mapping orbit (HAMO) imaging data (Roatsch et al., 2012) with a ground resolution of $\sim 60 \mathrm{~m} /$ pixel. Small areas were measured on higher resolved ( $\sim 20 \mathrm{~m} / \mathrm{pixel})$ imaging data from Dawn's low altitude mapping orbit (LAMO; Roatsch et al., 2013). In order to identify degraded craters with muted topography, we also used slope maps and maps of shaded relief.

The errors are calculated from the measured counting statistics and thus do not incorporate systematic errors such as the model of early Solar System bombardment history (see also Section 2.3).

For each measurement we present a topographic map and a cumulative crater plot including surface model ages. The fitted production function is drawn as dashed line except for the range used to fit the measured crater distribution, where it is drawn solid. In most cases we plot a randomness analysis above the crater plot. The measured craters are analyzed for the degree of random spatial distribution. Craters are considered to be randomly distributed, if their size bins fall within $\pm 3 \sigma$ (grayish zone). The randomness analysis is based on the SDAA (standard deviation of adjacent area) algorithm (Michael et al., 2012).There is also complementary information in the SOM (Section 2) with a crater map and differential crater plot with differential fit for surface age determination and the lunar equilibrium distribution for small craters (Neukum and Dietzel, 1971; Neukum, 1984). The term small craters is used for crater sizes $<\sim 10 \mathrm{~km}$, whose frequency distribution shows a steep slope of about -3 cumulative $(-4$ differential) in the used production function.

\subsubsection{Rheasilvia basin}

The Rheasilvia basin (Jaumann et al., 2012) was observed before Dawn arrived at Vesta by the Hubble Space Telescope (Thomas et al., 1997). The general findings based on Hubble data were confirmed by the Dawn mission (Jaumann et al., 2012; Schenk et al., 2012) although the basin has now been found to be a bit bigger and deeper than estimated in previous work. Vesta is probably the chief source of HED meteorites (e.g. Binzel and Xu, 1993), which can be traced back to the collisional family of Vesta (Nesvorný et al., 2008). Rheasilvia is thought to be the youngest impact event of global scale and it apparently contributed large amounts of material to replenish the Vestoid population. Thus, the age of Rheasilvia (Fig.5) puts constraints on the characteristics of the Vesta family (Marzari et al., 1996; Nesvorný et al., 2008) and our understanding of the dynamical evolution of asteroid collisional families in general. 

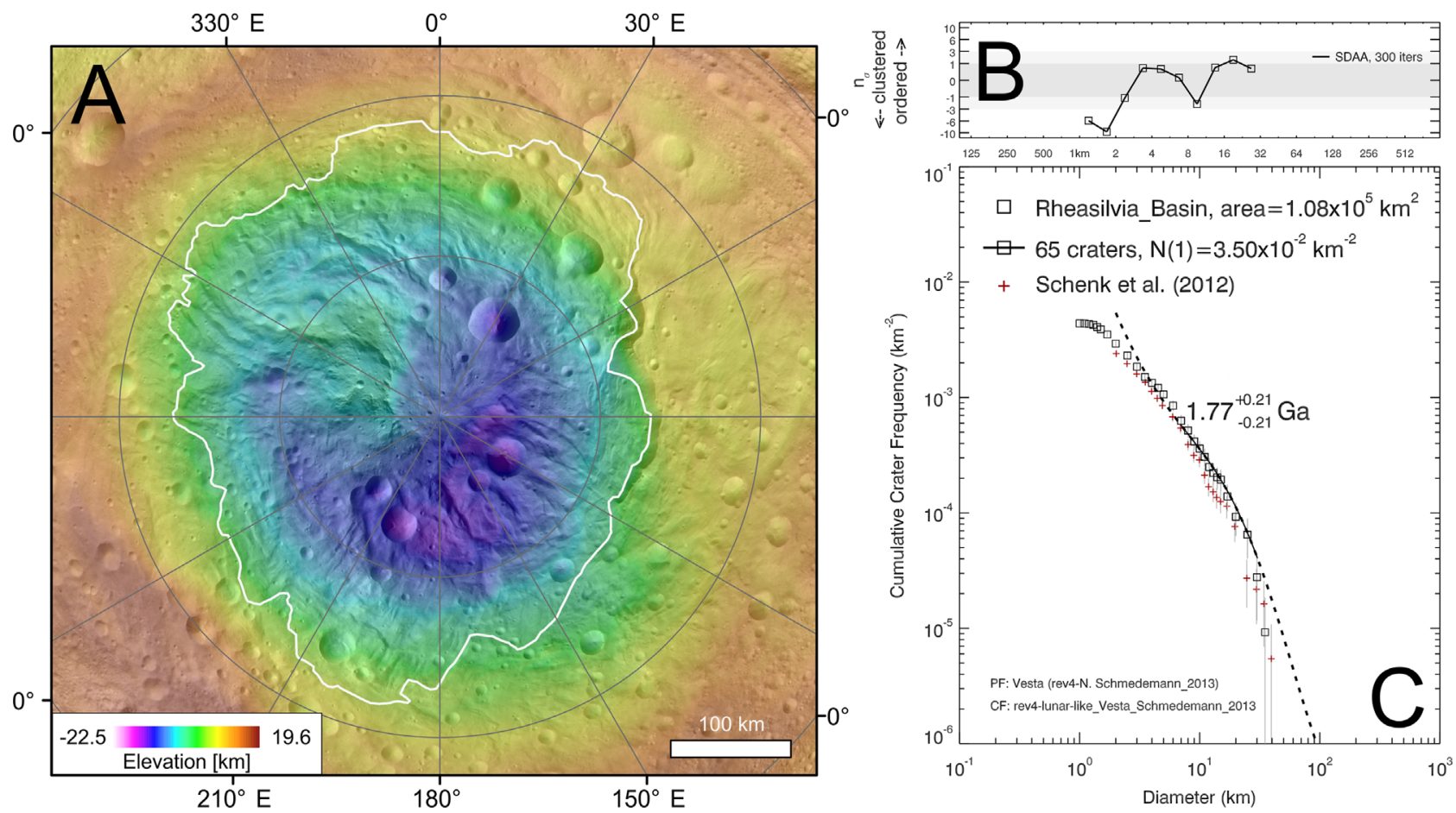

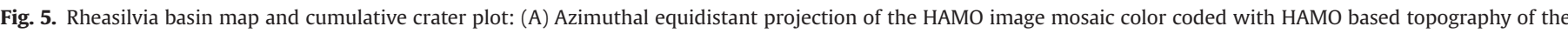

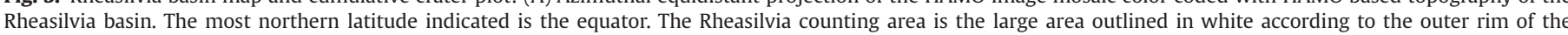

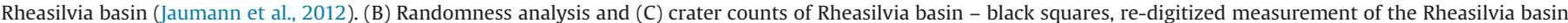
by Schenk et al. (2012) - red '+' signs.

The age of the Rheasilvia basin was estimated to be about $1 \mathrm{Ga}$ from crater measurements on the basin floor (Marchi et al., 2012c; Schenk et al., 2012). However, Rheasilvia ejecta on the floor of the older and smaller Veneneia basin (Jaumann et al., 2012) were dated by Schenk et al. (2012) at about 2 Ga. Assuming both crater counts by Schenk et al. (2012) are reasonable, that implies an earlier formation of Rheasilvia by roughly $1 \mathrm{Ga}$ and a resurfacing of the Rheasilvia basin floor such that it indicates a younger crater retention age. Either way, the formation age of the Rheasilvia basin is severely underestimated by Marchi et al. (2012c) and Schenk et al. (2012).

Mainly because of the Rheasilvia basin, Vesta is slightly out of equilibrium with a Maclaurin spheroid (Thomas et al., 1997) as determined from Vesta's principal dimensions where the equatorial radius is a little too large in relation to the polar radius to be in equilibrium (also Russell et al., 2012). However, it is probable that Vesta's lithosphere supports this inequity as can be inferred from the observed steep slopes at the crater rim of Rheasilvia, indicative of intact bedrock (Jaumann et al., 2012). On the other hand, loose regolithic material close to the surface of Vesta will likely follow the existing gravitational slope, whenever mobilized, for example by nearby impacts and move into the gravitational lows (Otto et al., 2013).

The measured crater size-frequency distribution follows closely the crater production function. The spatial crater distribution shows no significant clustering in the diameter range that has been used for the age determination. The measurement is well below the lunar equilibrium distribution for small lunar craters (SOM Fig. S4, Section 2.1.1), and thus should not suffer from effects of crater saturation. Our surface model age for the geologic unit of the Rheasilvia basin is $1.8 \pm 0.21 \mathrm{Ga}$ (Fig.5 panel C). Although our measurement is similar to Schenk et al. (2012) our derived model age is higher, primarily because of the different production functions used by both groups. But our data points also plot slightly higher. For comparison, we also show the re-digitized data by Schenk et al. (2012) in Fig. 5 (red crosses). Because the Rheasilvia basin is topographically about $9 \%$ below the reference sphere of Vesta, the respectively corrected crater sizes and size of the counting area are smaller than actually measured values. The correction results in a slight up-shift of crater frequencies, which could be one factor why our measurement is slightly above theirs. Different area outlines and crater identifications may be further sources of differences. We do not claim that our model age for the Rheasilvia floor closely corresponds to the Rheasilvia formation age, since, the Rheasilvia floor is a rather inhomogeneous surface unit, containing a large quantity of younger impact ejecta and showing strong indications for widespread downslope mass wasting (Otto et al., 2013). Although most of this mass wasting may have occurred shortly after basin formation (Schenk et al., 2012), repeated reactivation in more recent times is expected (e.g. due to impacts into the sloped basin walls). Mass wasting effectively destroys the cratering record and results in younger surface ages. At the central peak of the basin, a small area much older than $\sim 1.8$ Ga (see next section) can be found. The derived model age for the Rheasilvia basin may be interpreted as an approximate time at which the last major mass wasting inside the basin occurred. The true basin age is better indicated by the related ejecta blankets as well as the age of the central peak. These topographically high-standing areas should be less affected by mass wasting.

\subsubsection{Top of central peak of Rheasilvia}

Due to its positive topography, the area atop of Rheasilvia's central peak (Fig. 6) is not prone to be covered by mass wasted material. It thus offers a good possibility, of showing the formation age of the basin. This measurement is complemented by measurements in the northern hemisphere of Vesta, which 

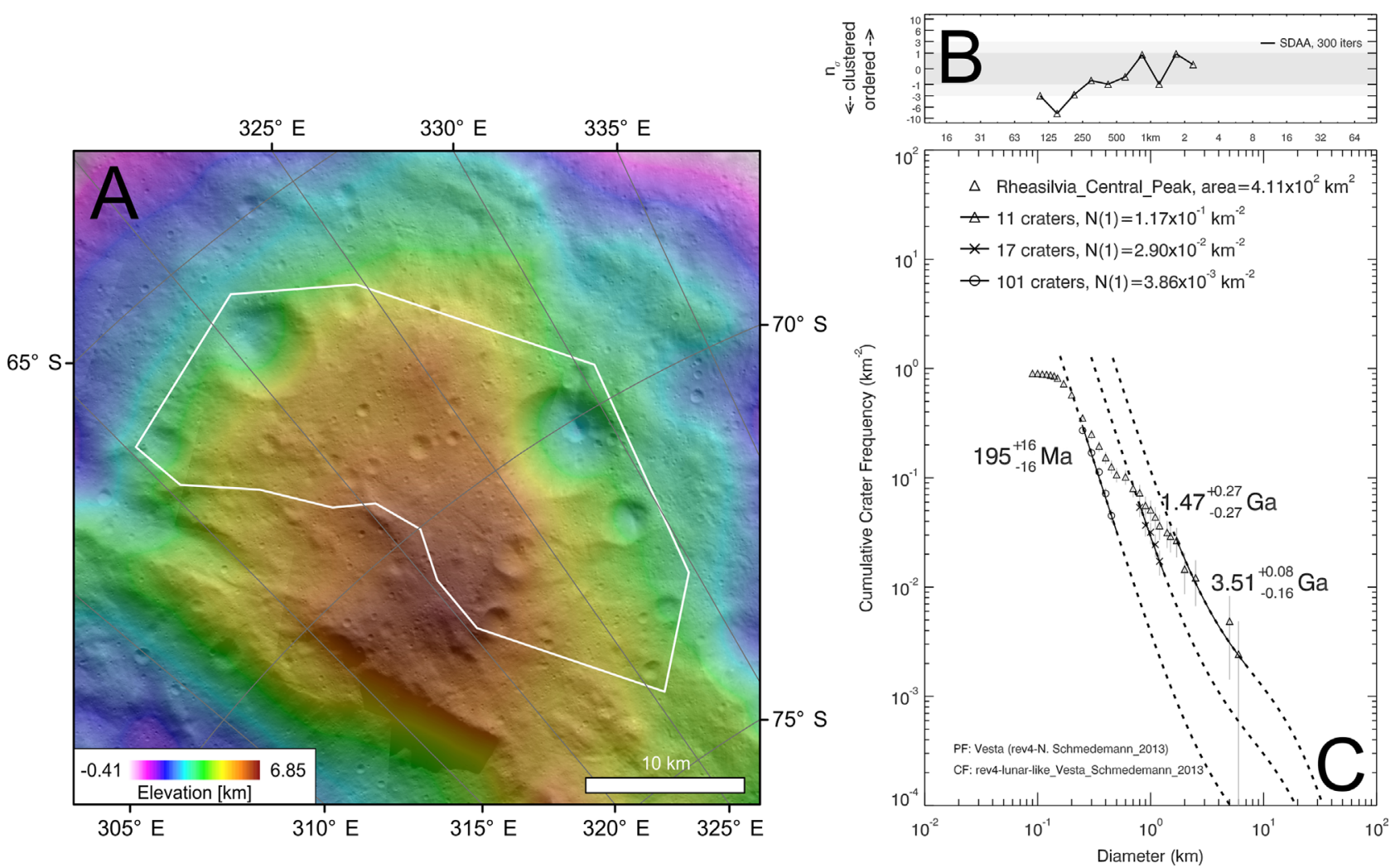

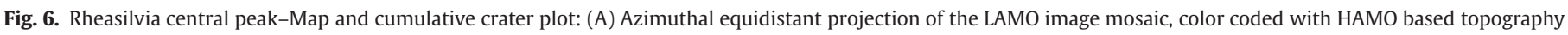

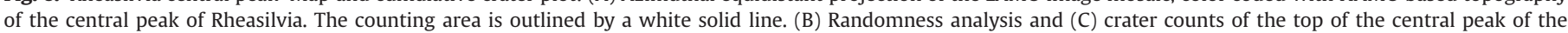
Rheasilvia basin - triangles, older resurfacing - crosses, younger resurfacing - circles.

could have been tectonically influenced by the South Pole basin formation (Bowling et al., 2013; Ivanov and Melosh, 2013) and the Rheasilvia ejecta blanket close to the crater Oppia (Le Corre et al., 2013).

Although selected for its expected low probability for being resurfaced due to mass wasting processes, this area shows a resurfaced crater distribution below $1.7 \mathrm{~km}$ crater size. 11 larger craters indicate a surface model age of $3.5_{-0.16}^{+0.08} \mathrm{Ga}$. These craters follow the production function reasonably well and suggest a reliable estimate of the surface model age. The found model age is significantly older than the average age of the whole Rheasilvia basin (previous area, Section 3.2.1). Since the central peak was formed together with the basin, the derived age of $\sim 3.5 \mathrm{Ga}$ is a lower threshold for the formation age of the Rheasilvia basin. It is a lower threshold, because later impact cratering could have reset the surface age at this area. Crater frequencies in the range of $0.8-1.3 \mathrm{~km}$ have been resurfaced by a younger event, which we dated to $1.5 \pm 0.27 \mathrm{Ga}$. Given the error bars this age could be thought to be contemporary with the average age of the whole Rheasilvia basin $(1.8 \pm 0.21 \mathrm{Ga})$. In the diameter range of 0.25 $0.5 \mathrm{~km}$ we may have indication for an even younger resurfacing event that ceased its activity $200 \pm 16 \mathrm{Ma}$ ago. These later events may have their source in seismic shaking/ejecta blanketing due to other impacts, probably in the vicinity of the counting area, which destroyed or covered predominantly smaller craters and affected larger craters to a lesser degree. The measured spatial crater distribution shows no significant clustering in the diameter range used for the age determinations. Our base age of $\sim 3.5 \mathrm{Ga}$ is derived from a crater distribution marginally above the lunar equilibrium (see SOM Fig. S5, Section 2.12) and thus may be influenced by crater saturation effects. In this case, the derived age is only a lower limit.

\subsubsection{The Tuccia quadrangle - Ridge and Groove Terrain}

The Tuccia quadrangle (Fig. 7) borders the Vestalia Terra unit in the north and in the south partly to the central peak of the Rheasilvia basin between $180^{\circ}$ and $270^{\circ} \mathrm{E}$. The slopes of Tuccia cover a difference in elevation of about $40 \mathrm{~km}$ over a distance of about $150 \mathrm{~km}$ at an average slope of about $15^{\circ}$. We measured crater frequencies in high resolution in the southern part of the Tuccia quadrangle, which belongs to the Rheasilvia basin formation (Jaumann et al., 2012). The Tuccia quadrangle geologic mapping is detailed by Kneissl et al. (2014), which is the source for the presented crater count.

The measured age of the Tuccia quadrangle confirms that larger subsections of the Rheasilvia basin show similar surface model ages to the entire basin area. For the counting area in the Tuccia quadrangle we derive $1.9 \pm 0.22 \mathrm{Ga}$, which is within the error bars the same age as the entire basin $(1.8 \pm 0.21 \mathrm{Ga})$. It is also similar to the older resurfacing age on top of the Rheasilvia central peak $(1.5 \pm 0.27 \mathrm{Ga})$. In the fit range between 2.5 and $35 \mathrm{~km}$ crater size, we observe a good agreement between the measured crater sizefrequency distribution and the production function. In this diameter range, a transition occurs in the slopes of the production function, which makes this measurement important to judge the quality of the scaling procedure we performed to convert the lunar production function to the impact conditions on Vesta. A similarly good result over the same slope transition feature can be observed in the dataset for Lutetia (SOM Fig. S3, Section 1.3). As shown in Fig. S6 (SOM) the measurement from the Ridge and Groove Terrain of the Tuccia quadrangle plots well below the lunar equilibrium distribution for small craters and the analysis for spatial randomness did not detect significant clustering for craters in the diameter range that is used. As outlined before, we interpret the age of about $1.9 \mathrm{Ga}$ as an age estimate for the last major 

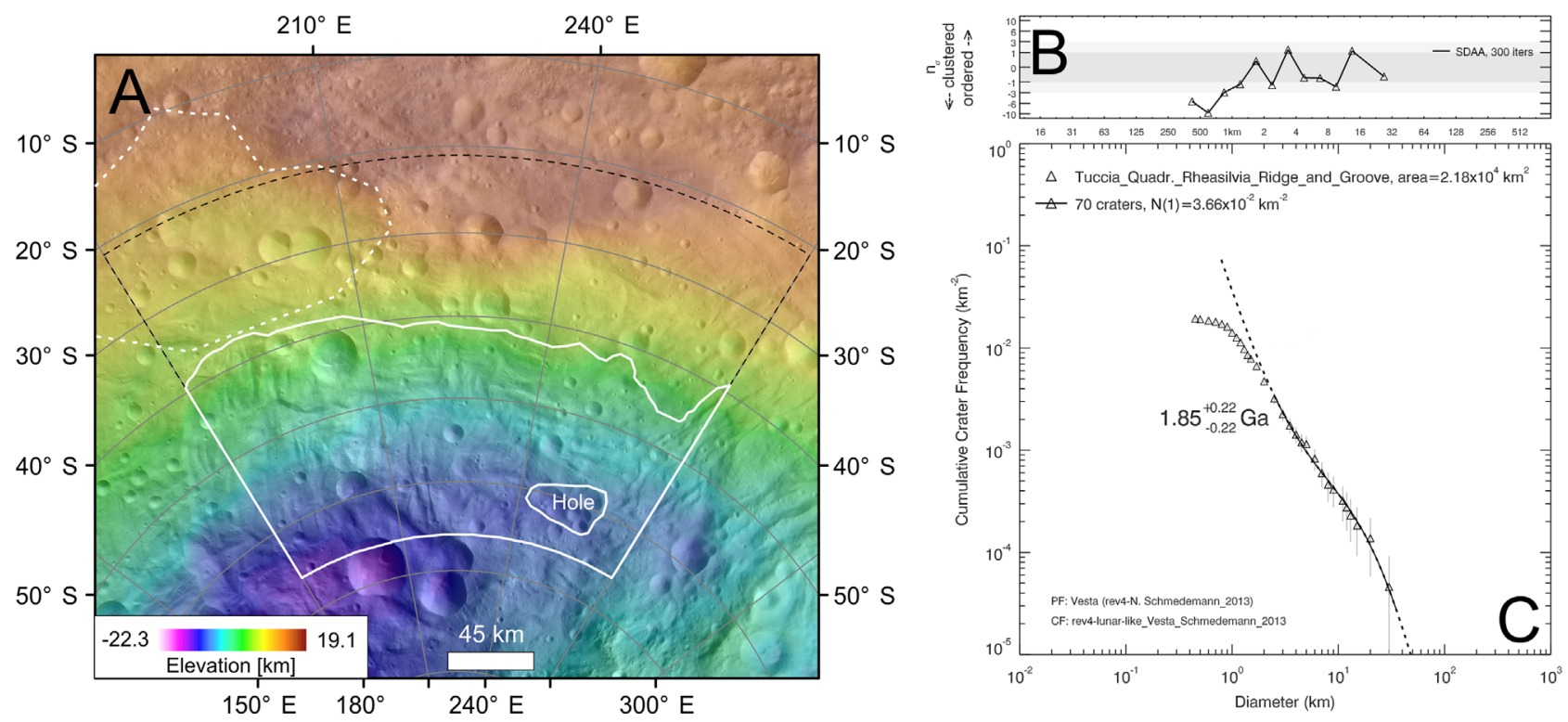

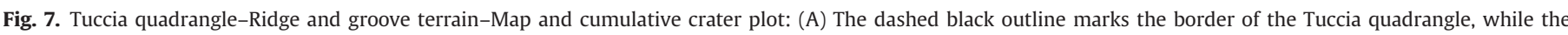

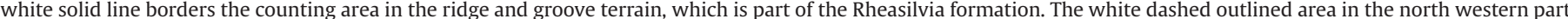

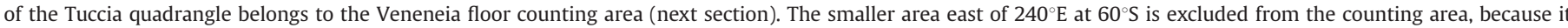

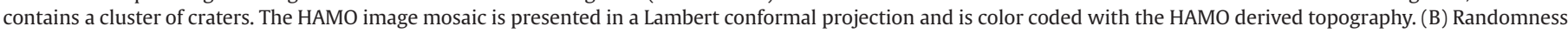
analysis and (C) crater counts of Ridge and Groove terrain in the Tuccia quadrangle - triangles.

resurfacing in this region. In the corresponding differential plot (SOM Fig. S6, Section 2.1.3), the crater distribution is approximated with two different isochrones, younger and older than the surface model age from the cumulative plot. This could be interpreted as two resurfacing events inside the counting area, with the cumulative model age being an averaged result.

\subsubsection{Floor of the Veneneia basin}

Veneneia is the smaller and older of the two prominent basins in the southern hemisphere of Vesta (Jaumann et al., 2012; Schenk et al., 2012). According to Schenk et al. (2012), its floor is heavily resurfaced by the Rheasilvia impact and therefore the derived surface age of Veneneia may only be a lower limit for the basin formation age. We elected to use only the eastern part of the Veneneia basin as the counting area, because the morphology in the west appears to be different from the east (Fig. 8). For instance, the west contains morphologically muted circular depressions, which are absent in the east of the floor of Veneneia. Thus, it is possible that after the formation of Veneneia, resurfacing processes like the Rheasilvia impact did not act equally over the part of the Veneneia basin not superposed by Rheasilvia. Due to its proximity to the larger and stratigraphically younger Rheasilvia basin, it is challenging to obtain a clear formation age for Veneneia from measurements of its basin floor covered by Rheasilvia ejecta. Theoretically, the age of Veneneia can be determined much better from areas specifically altered by the Veneneia impact event, such as the Saturnalia Fossae formation (Jaumann et al., 2012) in the northern hemisphere of Vesta. However, the measurement of sharp rimmed craters on top of the Rheasilvia ejecta blanket inside the Veneneia basin should give a lower threshold for the age of the Rheasilvia ejecta blanket.

For craters in the size range of $7-35 \mathrm{~km}$ diameter, we find a reasonable fit between the measured crater distribution and the production function. From this fit we derive a model age of $3.1_{-0.53}^{+0.2}$ Ga (Fig. 8). Smaller craters appear to be less frequent than expected from this isochron. Under the assumption, that this deficiency is caused by a resurfacing event, we made a resurfacing correction for the size range between 2 and $6 \mathrm{~km}$ diameter and found a resurfacing model age of $2 \pm 0.18 \mathrm{Ga}$. Based, on the test for spatial randomness, we find a slight clustering of craters in the range of 2 $4 \mathrm{~km}$ diameter, so it is possible that this diameter range is contaminated by secondary craters or is not uniformly resurfaced. Nevertheless, the crater distribution does follow the expected production function and shows no indication of an extraordinarily steep slope. Other crater sizes, which we used for age determination, appear to be randomly distributed as expected for an isotropic bombardment. The crater frequency below $10 \mathrm{~km}$ diameter is well below the lunar equilibrium distribution for small craters (see SOM Fig. S7), so we do not expect to observe crater saturation effects. The model age of $\sim 3.1 \mathrm{Ga}$ for a Rheasilvia blanketed area further supports the hypothesis of a formation age of the Rheasilvia basin much higher than $1 \mathrm{Ga}$ and subsequent resurfacing events. Interestingly, although not inside the Rheasilvia basin, this area also shows evidence for resurfacing around $2 \mathrm{Ga}$ ago, which is similar to several other areas inside the Rheasilvia basin. For comparison we also show the re-digitized measurement of the Veneneia floor by Schenk et al. (2012) in Fig. 8.

\subsubsection{Heavily cratered Terrains North of the equator}

North of the vestan equator, many large and heavily eroded impact structures are visible. This is one of the most densely cratered areas on Vesta. We defined a large counting area around $90^{\circ} \mathrm{E} / 20^{\circ} \mathrm{N}$, which stretches about $40^{\circ}$ in latitude and about $160^{\circ}$ in longitude (Fig. 9). Many of the larger craters are muted in their morphology due to obliteration and ejecta blanketing by subsequent impacts. Interestingly, the floors of small craters of 20$30 \mathrm{~km}$ in diameter show absolute altitudes in the range of $18 \mathrm{~km}$ below the vestan reference ellipsoid. The floors of similar sized craters in other regions of Vesta only reach such low altitudes inside larger basins like Rheasilvia or the equatorial basin at $310^{\circ} \mathrm{E}$ longitude (Feralia Planitia). Furthermore, some craters appear to show hollow depressions around them.

From the cumulative crater distribution in panel $C$ of Fig. 9, we derive a model age of $3.8_{-0.15}^{+0.07} \mathrm{Ga}$ in the crater diameter range of $60-100 \mathrm{~km}$. For craters $<60 \mathrm{~km}$, the cumulative plot shows a step towards lower crater frequencies, which likely indicates a resurfacing event. For the resurfaced crater distribution, we applied a resurfacing correction (Michael and Neukum, 2010) in the 

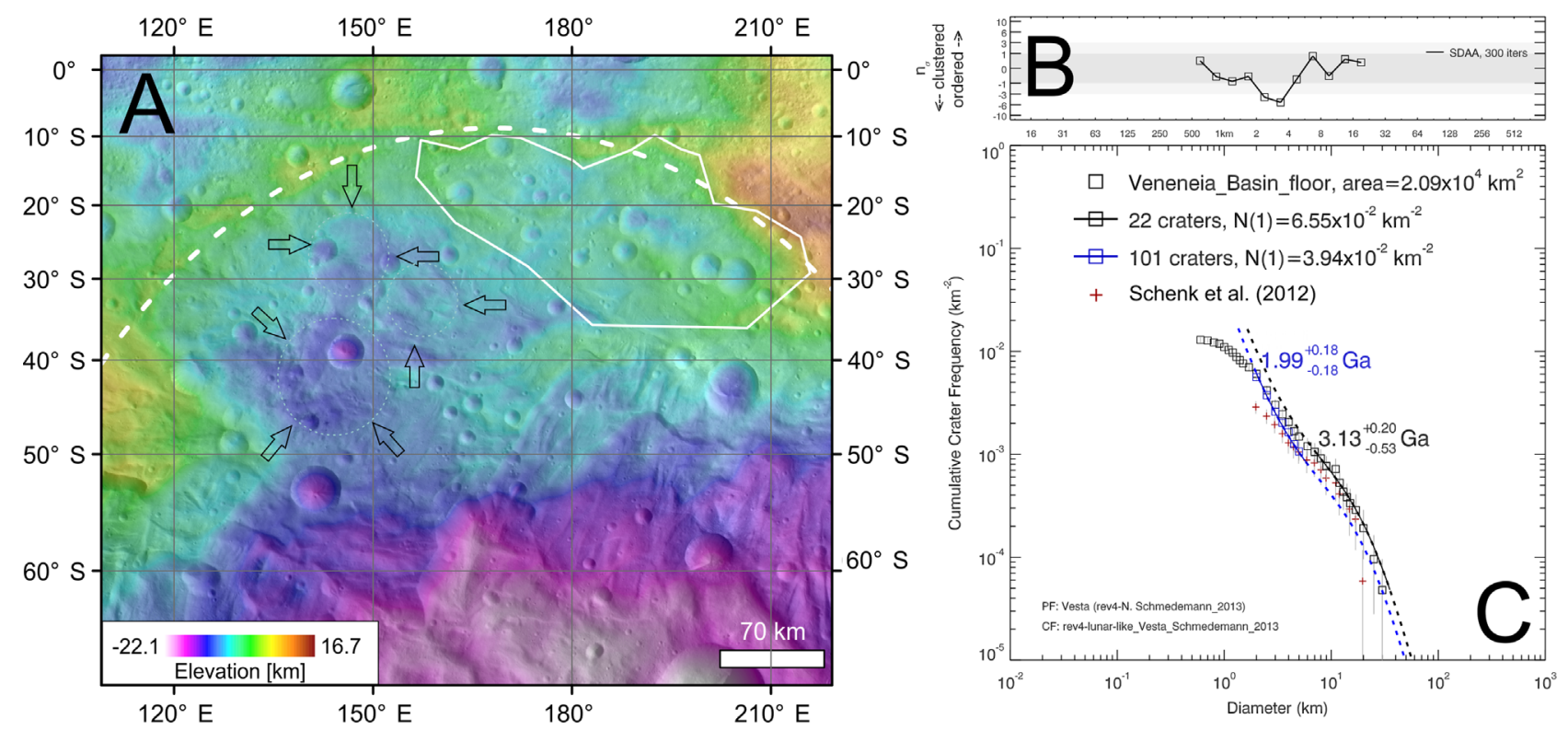

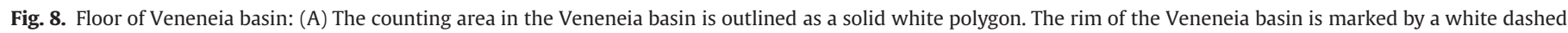

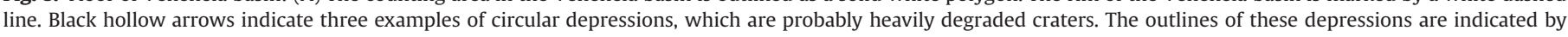

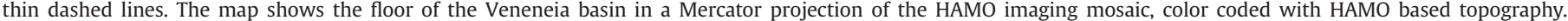

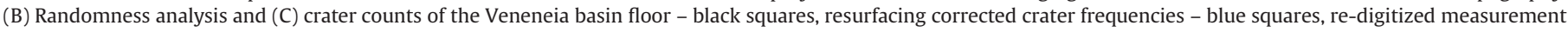
of the Veneneia basin floor by Schenk et al. (2012) - red '+' signs.

diameter range of $20-45 \mathrm{~km}$. The corrected measurement is consistent with a model age of $3.5_{-0.07}^{+0.05} \mathrm{Ga}$. This result is close to the model age derived from the top of the central peak of the Rheasilvia basin $3.5_{-0.16}^{+0.08} \mathrm{Ga}$. Thus, it might be possible that the observed resurfacing event is connected to the Rheasilvia basin formation. At crater sizes below $17 \mathrm{~km}$, we find an increase in crater frequencies above the resurfacing age isochron. The increase is small in the diameter range above $12 \mathrm{~km}$ but dramatically ramps up at $12 \mathrm{~km}$ until about $8 \mathrm{~km}$ diameter. At $4-6 \mathrm{~km}$ diameter the crater size-frequency distribution almost aligns with the $3.8 \mathrm{Ga}$ isochron, which is defined by the 4 largest craters. This observation of a steep crater size-frequency distribution in a limited size range may be consistent either with secondary cratering from the large basin formation in the southern hemisphere of Vesta and/or heavy bombardment by members of the Vesta family shortly after significant events of replenishment, also related to the basin formation. Since the lunar equilibrium for small craters is only valid below $\sim 10 \mathrm{~km}$ crater size, it cannot be used for the fit ranges we used for this area (SOM Fig. S8, Section 2.1.5). However, it is likely that the measured crater frequencies are close to equilibrium/saturation and thus, the found ages may only be lower limits for the actual surface ages. The spatial randomness test (Michael et al., 2012) shows no obvious crater clustering in either diameter range used for age determination. Interestingly, the diameter range containing the steep increase in crater frequency shows no heavy clustering either, which could imply distant secondary cratering as suggested by Bierhaus et al. (2012).

\subsubsection{North pole area}

Because this area is farthest from the impact site of Rheasilvia, one might expect to detect the highest surface ages on Vesta in the North Pole region. As Dawn departed from Vesta, it mapped the North Pole area with grazing incidence angles of sunlight, allowing for crater counting and age determination. Despite the difficult illumination conditions, Fig. 10 shows a couple of large craters of up to $80 \mathrm{~km}$ in diameter very close to the geometric antipode of
Rheasilvia $\left(121^{\circ} \mathrm{E} / 75^{\circ} \mathrm{N}\right)$. The possible antipodal effect by the Rheasilvia impact is discussed by Bowling et al. (2013).

The measured crater size-frequency distribution shows a high similarity to the crater distribution measured at the heavily cratered terrain (Section 3.2.5). Large craters in the range of $40-80 \mathrm{~km}$ indicate a model age of $3.8_{-0.06}^{+0.04} \mathrm{Ga}$. Smaller craters between 15 and $35 \mathrm{~km}$ diameter indicate a younger surface age, because crater frequencies in this range plot below the production function fitted to the large craters. We found a resurfacing model age of $3.5_{-0.04}^{+0.03} \mathrm{Ga}$. Given the high crater frequency in this area, it is possible that the derived ages are influenced by crater saturation: the derived ages should therefore be regarded as lower limit for the model age. Below $15 \mathrm{~km}$ crater size, the crater frequencies are steeper than the $3.5 \mathrm{Ga}$ isochron. The crater frequencies get close but do not reach the older isochron at $\sim 3.8 \mathrm{Ga}$. At about $7 \mathrm{~km}$ diameter, the crater distribution flattens into a roll-over. This behavior again might be caused by the influence of secondary/ Vestoid cratering. The randomness analysis did not reveal significant clustering in the size range used for the measurement. The age of the resurfacing event we found in this area is remarkably close to those we found in other areas on Vesta and which we linked to the formation of the Rheasilvia basin. This would suggest the Rheasilvia basin formation also influenced the North Pole area. The older base age may be connected to the formation of the Veneneia basin.

\subsubsection{Ancient crater at $250^{\circ} \mathrm{E} / 30^{\circ} \mathrm{N}$}

At about $250^{\circ} \mathrm{E}$ longitude, the southern edge of the North Pole area seems to superimpose an ancient large crater $(\varnothing \sim 180 \mathrm{~km})$, which has been altered by the Saturnalia Fossae formation ( Fig. 11; Jaumann et al., 2012). This crater is intriguing, because its northern half appears to be cut by a relatively flat and elevated terrain, which continues into the North Pole area. However, the northern crater rim is still visible in the higher flat terrain. We measured a relatively small area near the lowest part of the crater floor but at some distance from the steep slopes at the southern crater rim. We expect that the age of this area is connected to the 

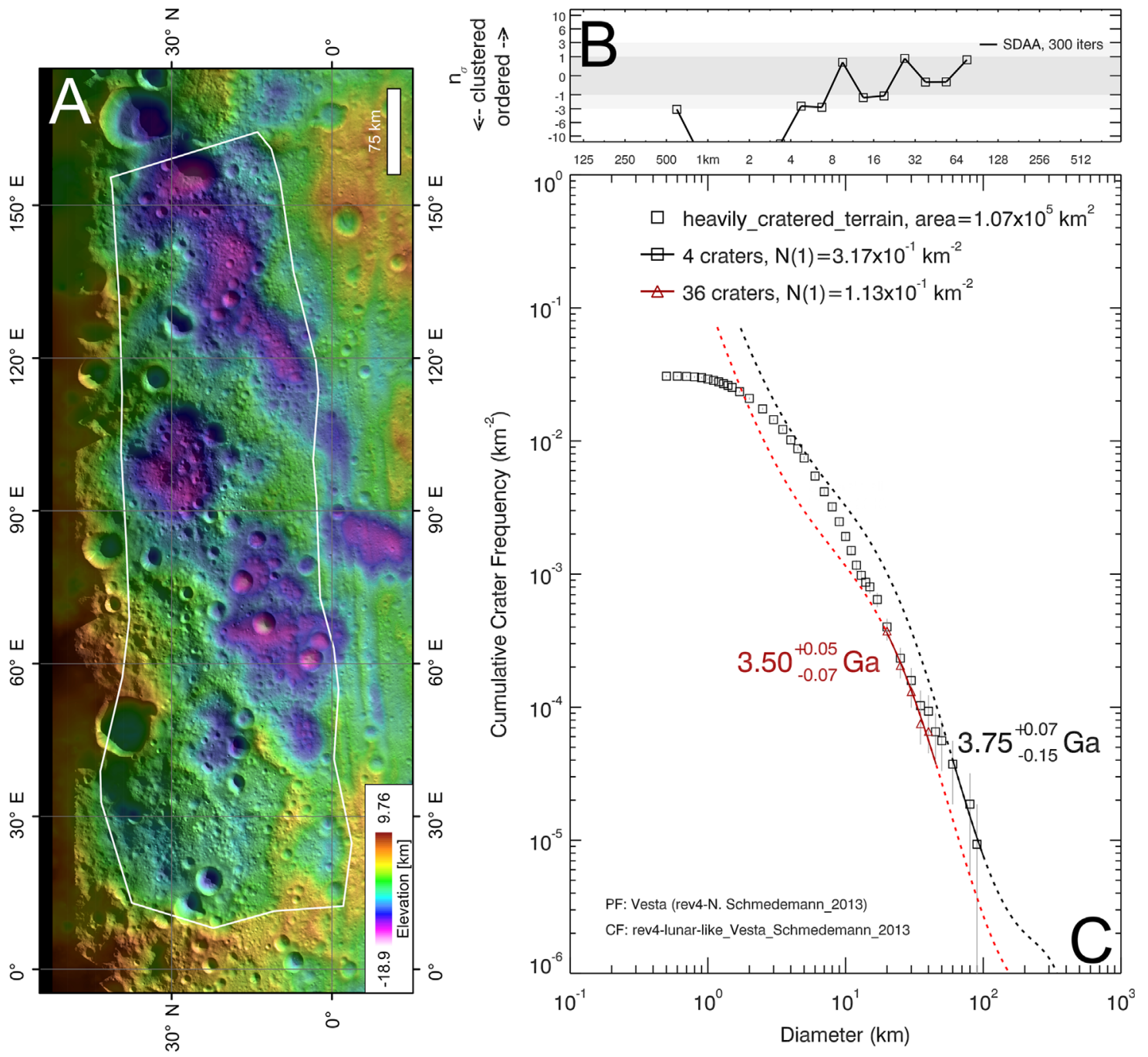

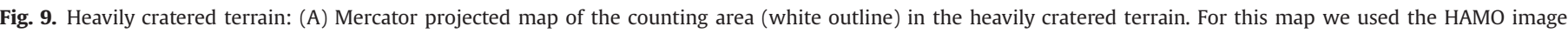

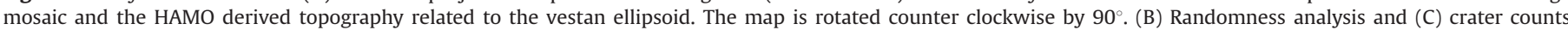
of heavily cratered terrain - black squares, resurfacing corrected crater frequencies - red triangles.

formation of Saturnalia Fossae and thus gives clues to the formation age of Veneneia. Furthermore, we measured two small areas atop that elevated terrain ("Elevated Terrain North" (ETN) and "Elevated Terrain South" (ETS), Fig. 11). We also measured two larger areas on lower resolution HAMO data, in order to crosscheck our results from the high resolution LAMO measurements. The HAMO measurements (Fig. 11), one on the crater floor and the other atop the elevated terrain are very similar to each other. Both show a crater distribution similar to the measurements at the North Pole and heavily cratered terrain areas (Sections 2.3 .5 and 2.3.6) with a steep crater distribution between $\sim 7$ and $\sim 12 \mathrm{~km}$. If that steep distribution is caused by Vestoid cratering or Rheasilvia secondary craters, it is possible that the presented LAMO crater counts suffer from such contamination, although it was indicated neither in the crater plots (Fig. 11) nor as clustered craters in the spatial crater distribution (SOM Figs. S11-S13, Section 2.1.7). In order to give a broader morphological characterization of this area, we also derived two topographic profiles starting at the two small counting areas on top of the elevated terrain, crossing each other in the area at the crater floor and extending further southward across the southern rim of the large ancient crater. The counting area on the crater floor is roughly at the intersection of the two profiles, at about $90-100 \mathrm{~km}$ in the profile. The profile shows a significant step where the elevated terrain cuts the crater floor. The step height varies but was measured at between $4000 \mathrm{~m}$ and $6500 \mathrm{~m}$. The reference height at the Saturnalia Fossae modified crater floor was set to an average level near the step. Stratigraphically, it is apparent that the elevated terrain is younger than the large crater. From tectonic relations, we believe that the Saturnalia Fossae formation on the crater floor is connected to the Veneneia impact structure (Jaumann et al., 2012), which makes the large crater older than Veneneia. Thus, after Vestalia Terra in which the crater formed, the crater is the oldest geologic structure in the area. Since the larger Rheasilvia impact is stratigraphically younger, it is possible that this area was already fractured by the Veneneia impact and the formation of Saturnalia and was later exposed to high levels of seismic activity during Rheasilvia's formation.

From the small area on the floor of the ancient crater we derive a surface model age of $3.8{ }_{-0.07}^{+0.04} \mathrm{Ga}$. This result is comparable to our results for the model age at the heavily cratered terrain and the North Pole. The two small areas atop of the elevated terrain show younger ages. For the ETN area we derive $3.6_{-0.23}^{+0.09} \mathrm{Ga}$ and for the ETS area we find $3.6_{-0.05}^{+0.04} \mathrm{Ga}$. These ages are similar to results from other areas we would link to the formation of the Rheasilvia basin. Both of the latter two areas are very similar to each other but appear significantly 

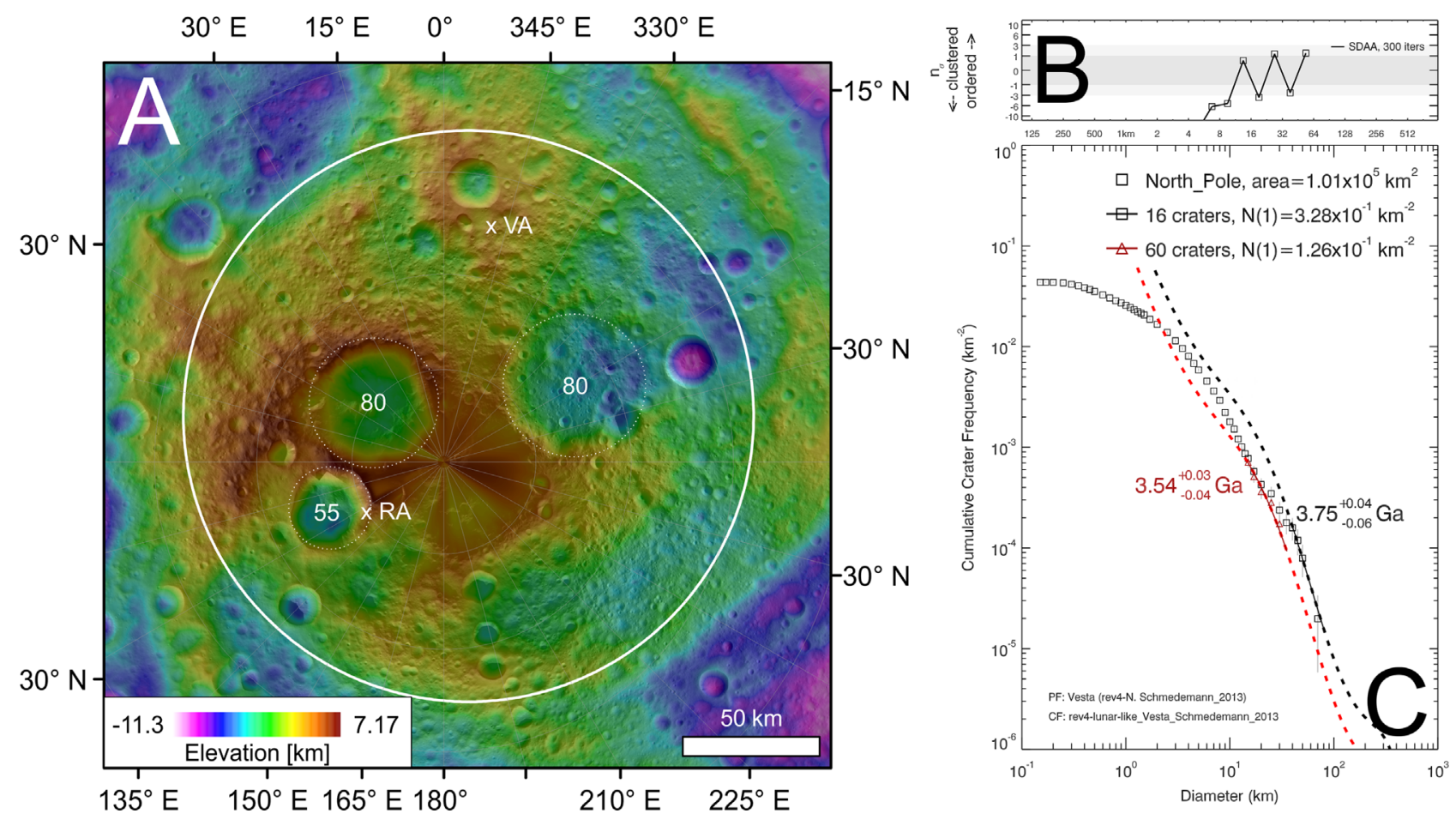

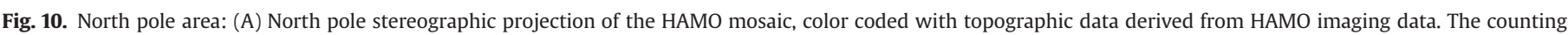

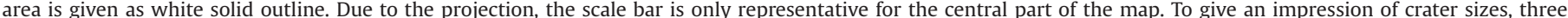

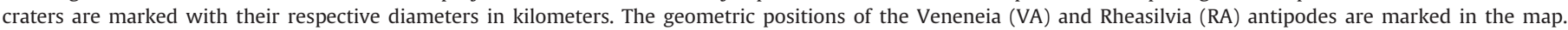
(B) Randomness analysis and (C) crater counts of North pole area - black squares, resurfacing corrected crater frequencies - red triangles.

younger than the area at the crater floor. This can easily be seen in the crater plot of Fig. 11. All of the given ages imply crater frequencies above the lunar equilibrium distribution for small craters (SOM Figs. S11-S13, Section 2.1.7). Thus, these model ages may only be considered as lower estimates for the true surface model ages. However, the younger nature of the elevated terrain is not supported from the two HAMO measurements, which show a high similarity to each other. From craters larger than $\sim 12 \mathrm{~km}$ we date the elevated terrain with 3.6 $6_{-0.06}^{+0.04} \mathrm{Ga}$ and the crater floor with $3.6_{-0.07}^{+0.05} \mathrm{Ga}$. These ages are highly similar to the results from the LAMO measurements in the elevated terrain. It is not obvious, why the HAMO measurement in the elevated terrain gives a significant higher crater frequency than the LAMO measurement for craters $<\sim 7 \mathrm{~km}$. The reason could be the difficult illumination condition in the LAMO data. But it is also possible that area location and size may also play some role.

Over the diameter ranges, which we used to fit the production function to the measurement, we find good agreement between the measured size-frequency distribution and the shape of the production function. At particular diameters however, in each measurement the crater size distribution falls below the production function. The small area on the crater floor shows such a kink at $\sim 2 \mathrm{~km}$ diameter and continues towards smaller crater sizes with a distribution flatter than the lunar equilibrium. Thus, the distribution is indicative for an ongoing resurfacing possibly caused by various agents such as seismic shaking and ejecta blanketing of neighboring impacts. The ETN area shows its kink at $\sim 1.2 \mathrm{~km}$ it continues steeper than the size distribution from the crater floor. In fact, the crater distribution of the ETN area falls along the lunar equilibrium for small craters (SOM Fig. S12, Section 2.1.7). This is interesting, since it may imply that craters $>1.2 \mathrm{~km}$ are not in equilibrium and thus, the derived surface model age is more reliable. The ETS area (SOM Fig. S13, Section 2.1.7) shows a kink at $\sim 600 \mathrm{~m}$ diameter and continues with a shallow slope, similar to the one of the crater floor. This implies a similar conclusion as for the crater floor, i.e. this crater distribution is modified by ongoing resurfacing. For clarity and as an exception to other areas discussed in this section, the randomness analysis for the five areas is shifted to the SOM (Figs. S11S14, Section 2.1.7). The crater size bins which have been used for the age determination were sometimes not populated enough (minimum of 3 craters) to conduct a randomness analysis. In cases where there were enough craters, they show a random spatial distribution.

Both HAMO measurements appear to follow the $3.8 \mathrm{Ga}$ isochron from $\sim 3 \mathrm{~km}$ to $\sim 7 \mathrm{~km}$ crater size (SOM Fig. S14, Section 2.1.7). It may be possible that the crater distribution is actually in equilibrium and does not really follow the $3.8 \mathrm{Ga}$ isochron. From the presented data it is hard to distinguish between both cases. If the measurement is in equilibrium below $\sim 7 \mathrm{~km}$ this is an indication that the lunar equilibrium does not apply to Vesta but is vertically shifted to higher frequencies by a factor of about 2 or 3. Then, model ages of about $3.6 \mathrm{Ga}$ or less are well defined and no lower limits.

\subsubsection{Oppia ejecta blanket}

The two measurements of the Oppia ejecta blanket (Fig. 12) have already been presented by Le Corre et al. (2013). Here, we present updated model ages after application of the measurement correction to account for the projection error caused by the differences between the real topography and the used reference body. Furthermore, the already published data utilizes rev3 of the vestan production and chronology functions, where here we use rev4. Both versions are described in Sections 2.2 and 2.3 of this work.

Oppia is located immediately north of the rim of the Rheasilvia basin and thus likely formed within the Rheasilvia ejecta blanket. Craters which formed in the massive Rheasilvia ejecta should 
A

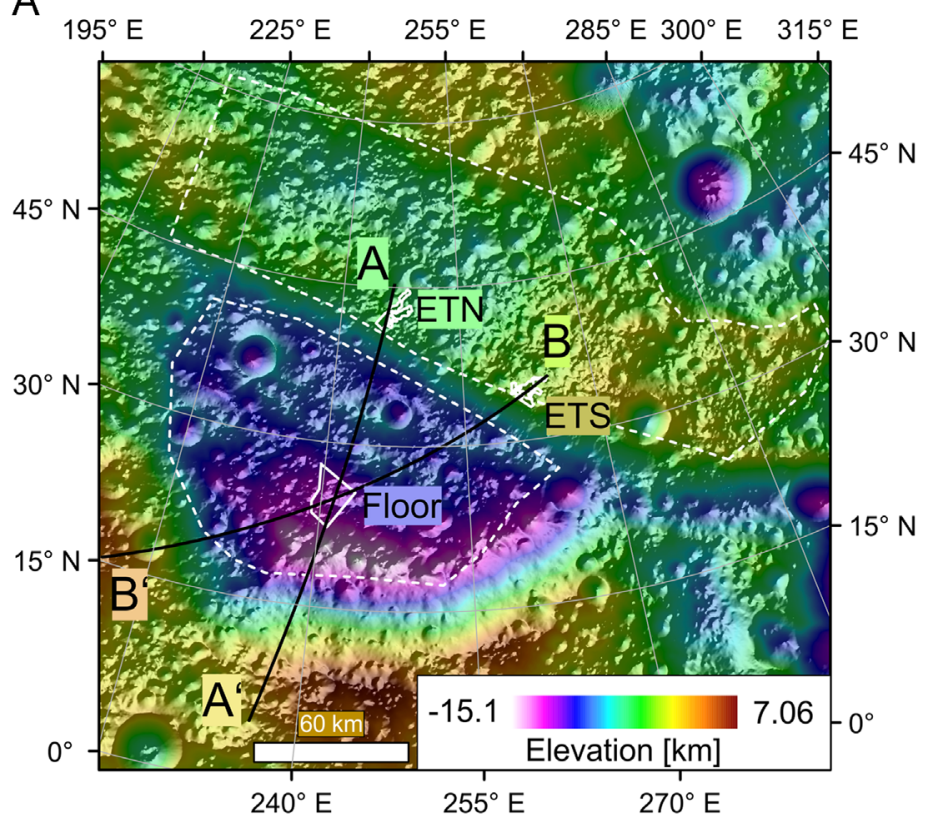

B

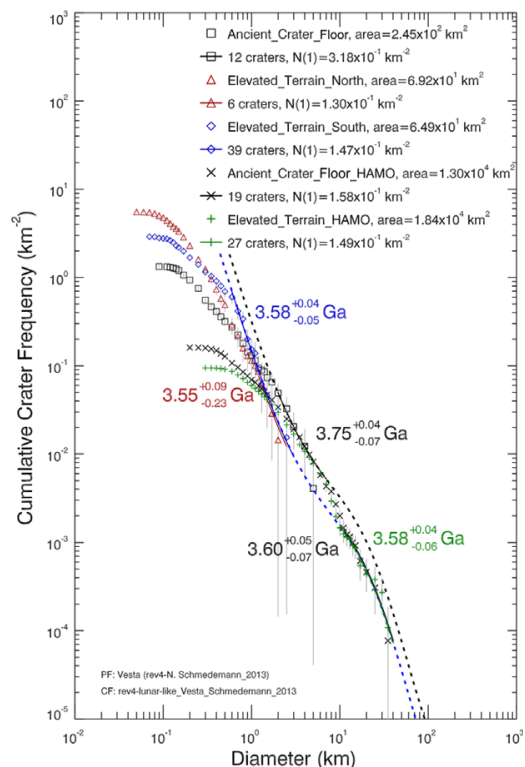

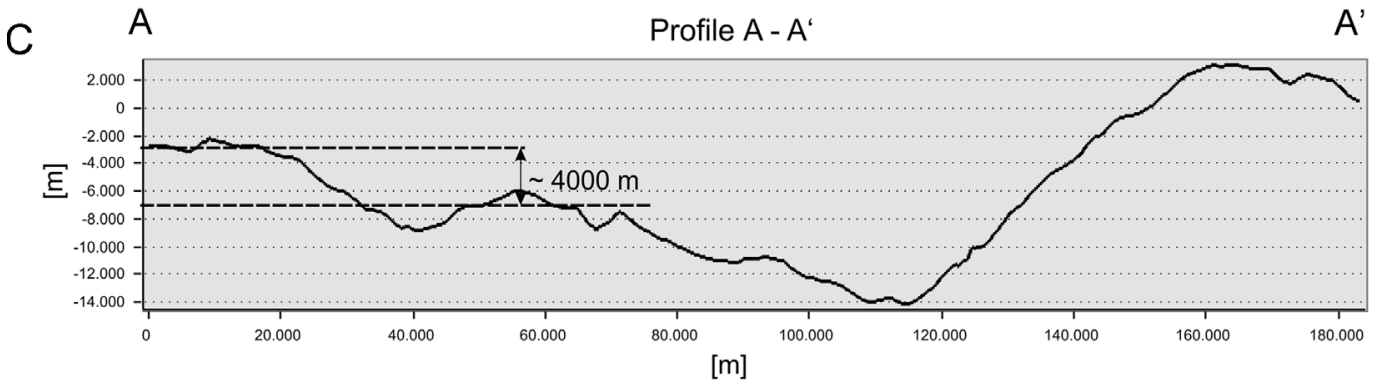

B

Profile B - B'

$\mathrm{B}^{\prime}$

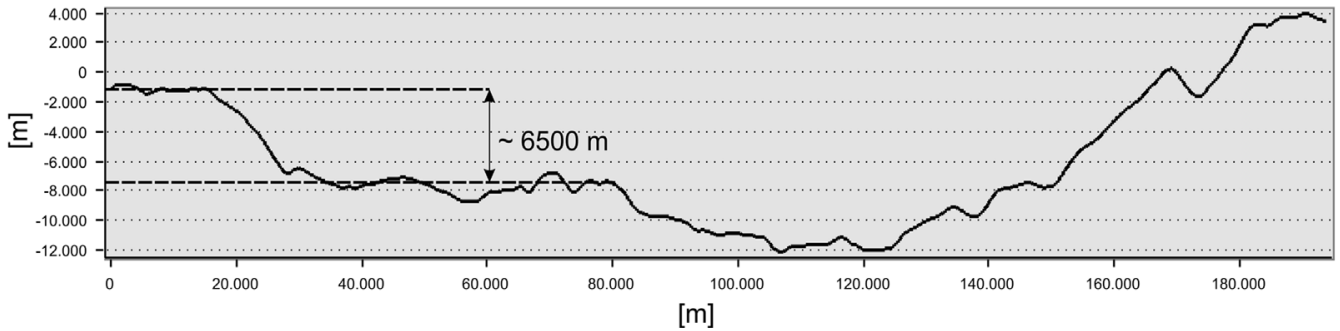

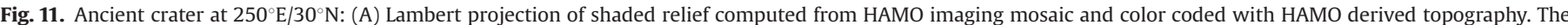

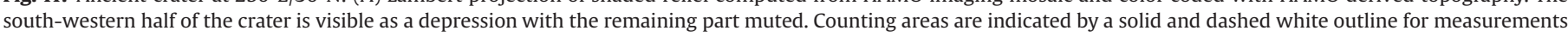

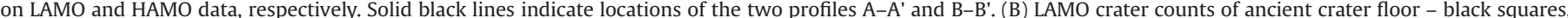

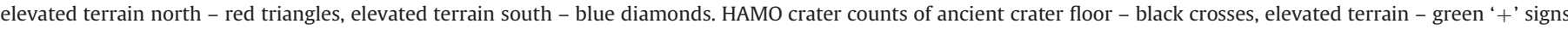
and $(\mathrm{C})$ topographic profiles A-A' and B-B' based on the HAMO DTM. Dashed lines approximate the average heights of the elevated terrain and the crater floor.

indicate the formation age of the Rheasilvia ejecta. Thus, dating Oppia ejecta, including stratigraphically underlying craters, might also provide information about the formation age of the Rheasilvia basin itself. We measured the Oppia ejecta blanket in one area north of Oppia (northern part), which is further away from Rheasilvia and a second area south of Oppia (southern part) closer to Rheasilvia.

The area in the northern part of the Oppia ejecta blanket reveals a crater distribution typical for a resurfacing event (Fig. 12). That is for a cumulative representation a depletion of crater frequencies below a certain diameter and a buildup of crater frequencies according to the production function when going towards smaller diameters. A differential crater plot shows a simple step towards lower frequencies for craters below a specific crater diameter (SOM Fig. S15, Section 2.1.8). The step size is a measure of the time difference between the older underlying unit and the younger ejecta blanket at the current surface. For the northern Oppia ejecta blanket we derive from craters $\geq 2 \mathrm{~km}$ a model age of $3.6_{-0.11}^{+0.06} \mathrm{Ga}$. This might be interpreted as the age of the Rheasilvia ejecta blanket in which Oppia formed, the resurfacing event being the emplacement of the Oppia ejecta. Le Corre et al. (2013) find a model age of $3.6_{-0.09}^{+0.05} \mathrm{Ga}$ for the same area. The formation age of Oppia is derived from the resurfaced part of the crater distribution (0.3-0.7 km diameter) and gives a model age of $320 \pm 24 \mathrm{Ma}$. Le Corre et al. (2013) find for this area a model age of $280 \pm 23 \mathrm{Ma}$, which is in agreement to the precision of the error 

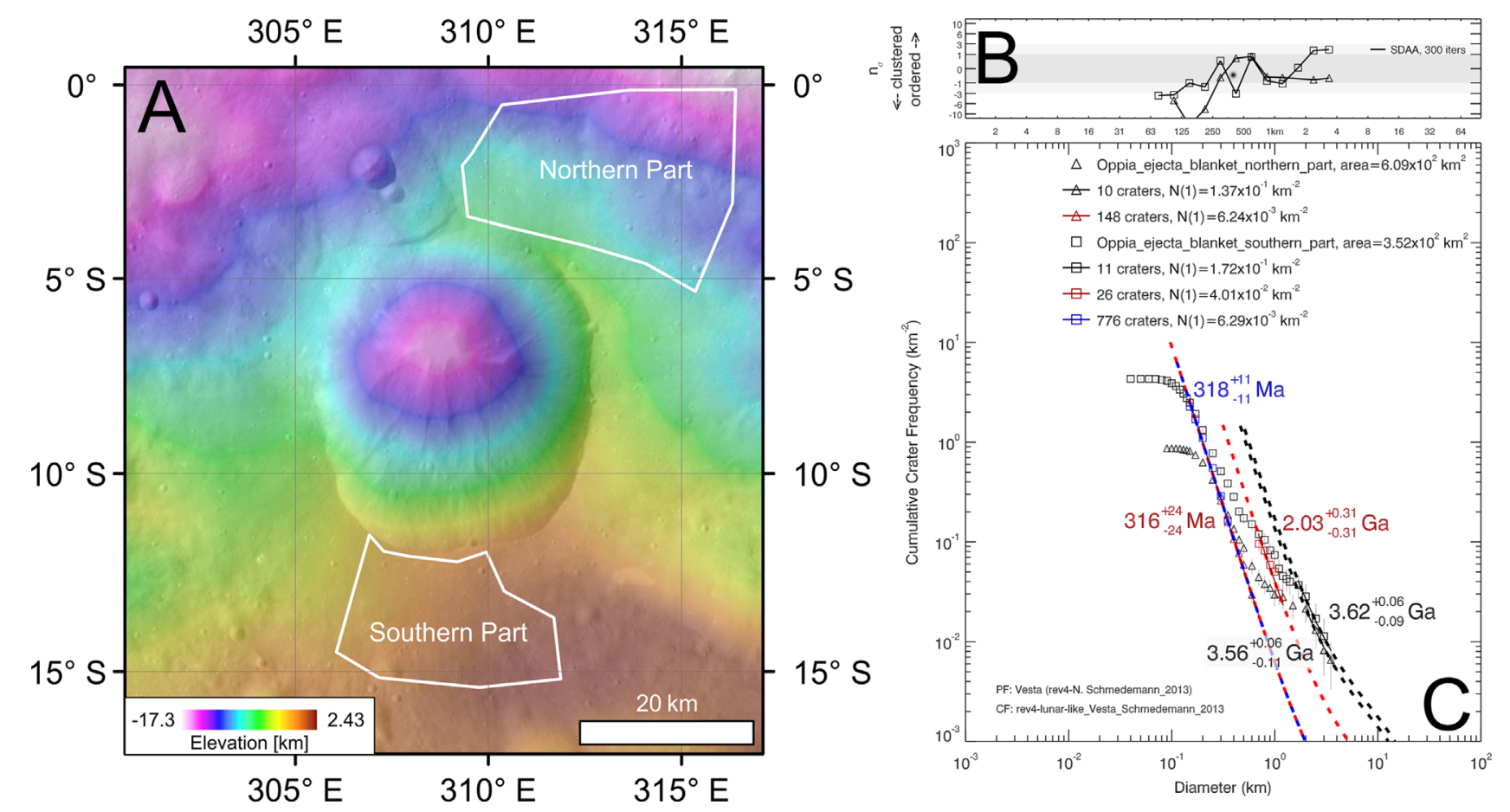

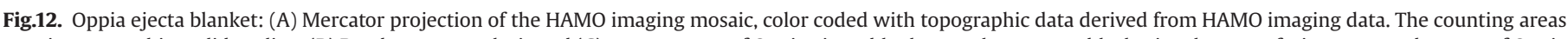

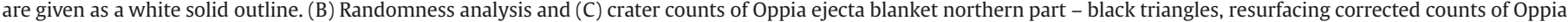

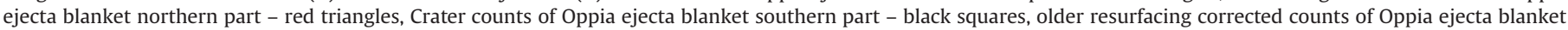
southern part - red squares, younger resurfacing corrected counts of Oppia ejecta blanket southern part - blue squares.

bars. Frequencies of craters $\geq 2 \mathrm{~km}$ plot very close to the lunar equilibrium distribution for small craters (SOM Fig. S15, Section 2.1.8). Thus, the found age may only be a lower limit.

The southern part of the Oppia ejecta blanket shows a more complicated crater size-frequency distribution, as it features an additional kink at about $1 \mathrm{~km}$ diameter. This kink may indicate another resurfacing event before the Oppia impact. For the model age defined by craters $\geq 1.7 \mathrm{~km}$ we find $3.6_{-0.09}^{+0.06} \mathrm{Ga}$. Le Corre et al. (2013) find a model age of $3.6_{-0.1}^{+0.06}$ Ga for the same area. In this work, we date the older resurfacing with $2 \pm 0.31 \mathrm{Ga}$, utilizing resurfacing corrected crater frequencies in the range of $0.7-1.2 \mathrm{~km}$. Le Corre et al. (2013) find for the older resurfacing in this area a model age of $1.8 \pm 0.3 \mathrm{Ga}$, also using a resurfacing correction (Michael and Neukum, 2010). The Oppia formation age is derived from the younger resurfacing event, which we date in this work to $320 \pm 11 \mathrm{Ma}$, utilizing resurfacing corrected crater frequencies in the range of $0.15-0.4 \mathrm{~km}$ diameter. Le Corre et al. (2013) find a model age of $270 \pm 9.3 \mathrm{Ma}$ for the younger resurfacing in this area. Thus, for the southern part of the Oppia ejecta blanket we see a slight deviation in the Oppia formation age between this work and Le Corre et al. (2013). The older resurfacing and the underlying ages of the Rheasilvia ejecta are the same within the error bars in both works, respectively. Frequencies of craters $\geq 1.7 \mathrm{~km}$ plot very close to the lunar equilibrium distribution for small craters (SOM Fig. S16, Section 2.1.9). Thus, the age of the Rheasilvia ejecta may only be a lower limit. Resurfaced crater frequencies are slightly below the lunar equilibrium and may also show a slight influence from saturation effects.

It is intriguing that the Rheasilvia facing section of the Oppia ejecta shows an additional resurfacing age of about $2 \mathrm{Ga}$, which is absent on the opposite side of the ejecta blanket. Vast areas inside the Rheasilvia basin show model ages of about $2 \mathrm{Ga}$ as well, as has been shown in the previous measurements. This could possibly indicate a significant resurfacing event inside the Rheasilvia basin around that time, which obliterated most of the previous cratering record inside the basin.

\subsubsection{Octavia ejecta blanket}

Model ages of the areas of the Octavia ejecta blanket presented here have also been shown by Le Corre et al. (2013). As outlined for the Oppia ejecta blanket (previous section) we update the already published age data by applying the same changes as for the Oppia measurements.

Octavia is of interest because it is located close to the northern rim of the Veneneia basin. Thus, this configuration is comparable with Oppia in relation to Rheasilvia and due to the distance of Octavia to Rheasilvia, the influence of Rheasilvia ejecta may be relatively limited. We therefore use measurements from the Octavia ejecta blanket, in order to date both the Octavia ejecta blanket and the Veneneia ejecta blanket. Octavia also features a landslide in its interior, which has not been further investigated in this work. The landslide indicates a geologic activity after the crater formation similar to what we expect from the Rheasilvia interior, but on much smaller scale.

The south-east counting area (Fig. 13) shows a resurfaced crater distribution, similar to the Oppia ejecta blankets. Craters $\geq 2.5 \mathrm{~km}$ can be well fitted with the vestan production function. We derive a model age of $3.7_{-0.09}^{+0.06} \mathrm{Ga}$. The measured crater frequencies in this diameter range are well above the lunar equilibrium distribution for small craters (SOM Fig. S17, Section 2.1.10). Thus, the derived age is only a lower limit for the Veneneia ejecta blanket in which Octavia presumably formed. Resurfacing corrected crater frequencies between 0.25 and $0.7 \mathrm{~km}$ crater size reveal a resurfacing model age of $390 \pm 28 \mathrm{Ma}$. This would be the formation age of Octavia. The respective crater frequencies are below the lunar equilibrium distribution for small craters (SOM Fig. S17, Section 2.1.10) and thus may not show significant influence from crater 

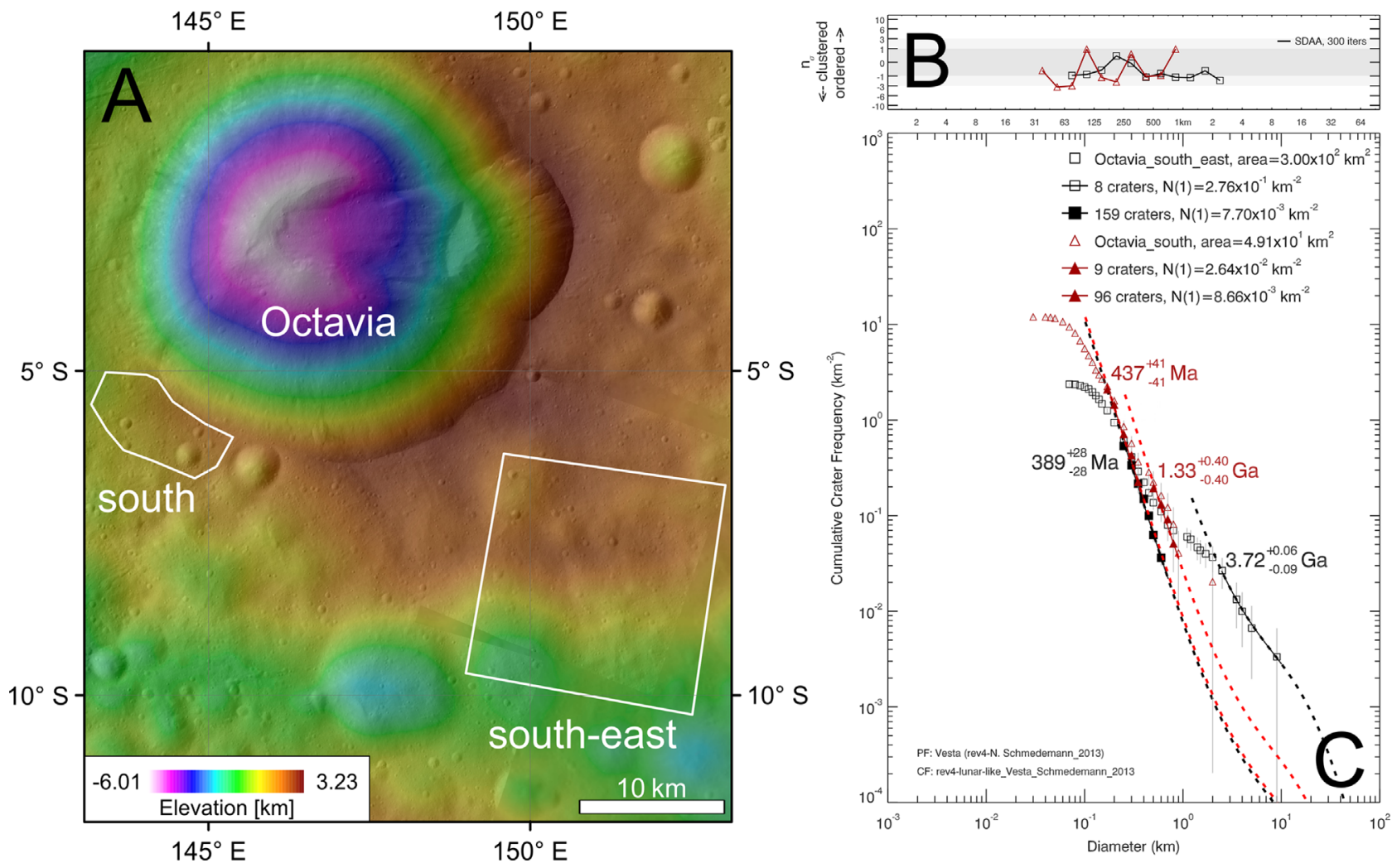

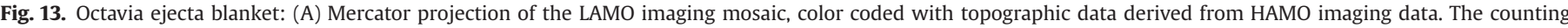

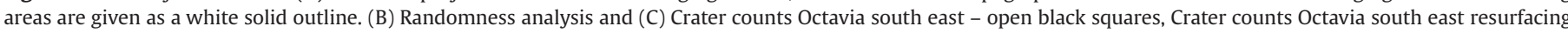
corrected - filled black squares, Crater counts Octavia south - open red triangles, Crater counts Octavia south resurfacing corrected - filled red triangles.

saturation effects. Le Corre et al. (2013) present a model age of $3.8_{-0.06}^{+0.04} \mathrm{Ga}$ and a resurfacing model age of $370 \pm 30 \mathrm{Ma}$, in agreement with our updated surface model ages.

The "Octavia_south" area (Fig. 13) is smaller than the "Octavia_south_east" area. It is also located closer to the crater rim of Octavia and thus probably shows fewer craters which survived the Octavia formation. From crater diameters in the range of $0.5-0.9 \mathrm{~km}$, we derive a model age of $1.3 \pm 0.4 \mathrm{Ga}$. This is significantly younger than the oldest age of the south-east area. We do not interpret this age as a possible formation age of the Veneneia ejecta, but rather the influence of another event that could be the emplacement of another ejecta blanket. The detected signal in the crater distribution is weak and should not be overinterpreted: it was not presented by Le Corre et al. (2013). We date the resurfacing event, which is likely connected with the Octavia formation with a model age of $440 \pm 41 \mathrm{Ma}$. In order to do that, we use resurfacing corrected crater frequencies in the range of 0.17 to $0.4 \mathrm{~km}$ diameter. For the corresponding dataset, Le Corre et al. (2013) find a model age of $360_{-38}^{+39} \mathrm{Ma}$, which is similar but slightly outside the error bars. The crater frequencies for both the underlying old age and the resurfacing model ages plot very close to the lunar equilibrium distribution for small craters (SOM Fig. S18, Section 2.1.11). Thus, the derived ages may only be lower limits.

\subsubsection{Ejecta blanket of Antonia crater}

Part of the eastern ejecta blanket of the Antonia crater has been counted and dated with rev3 of the lunar-like vestan crater production and chronology functions by Kneissl et al. (2014). In order to show the applicability of the lunar-like production function for Vesta, we present this measurement (Fig. 14; SOM Fig. S19, Section 2.1.12) with updated rev4 model ages. Antonia is an apparently young sharp-rimmed crater. Thus, its ejecta blanket has a low likelihood of being affected by subsequent resurfacing events, such as seismic shaking or ejecta blanketing by other nearby impacts. Antonia is an example of an asymmetric crater, typical for impacts on slopes (Jaumann et al., 2012) and it also features dark ejecta. The crater is detailed, for instance, by Kneissl et al. (2014).

The measured distribution of small craters atop the Antonia ejecta blanket is in remarkably good agreement with the lunar-like production function for Vesta. We fit the crater distribution between 0.06 and $0.3 \mathrm{~km}$ diameter and find a surface model age of $21 \pm 1.7 \mathrm{Ma}$. This would also be the formation age of the Antonia crater. For comparison, Kneissl et al. (2014) find a model age of $24 \pm 1.1 \mathrm{Ma}$ for this area using rev3 of the production and chronology functions.

\subsection{Summary on crater retention ages of key areas on Vesta}

Table 6 summarizes the results of all areas measured on Vesta and which are described in Section 3.2 "Surface Ages of Vesta". We present model ages not only for the rev 4 version of the lunar-like chronology but also give results for an alternative chronology based on the Late Heavy Bombardment (LHB) model, which is detailed by O'Brien et al. (under review). For the ages based on O'Brien's chronology model, we still use our lunar-like production function to fit the measured crater frequencies. From the lunar-like production function, we derive the respective $N(1)$ values (frequency of craters $\geq 1 \mathrm{~km}$ diameter). The determined $N(1)$ value is then projected to O'Brien's chronology function to convert the $N$ (1) value into a model age. More detailed tables are presented in the SOM (Table S1 for cumulative fits and Table S2 for differential fits, Section 2.2) including area sizes and $N(1)$ values. 

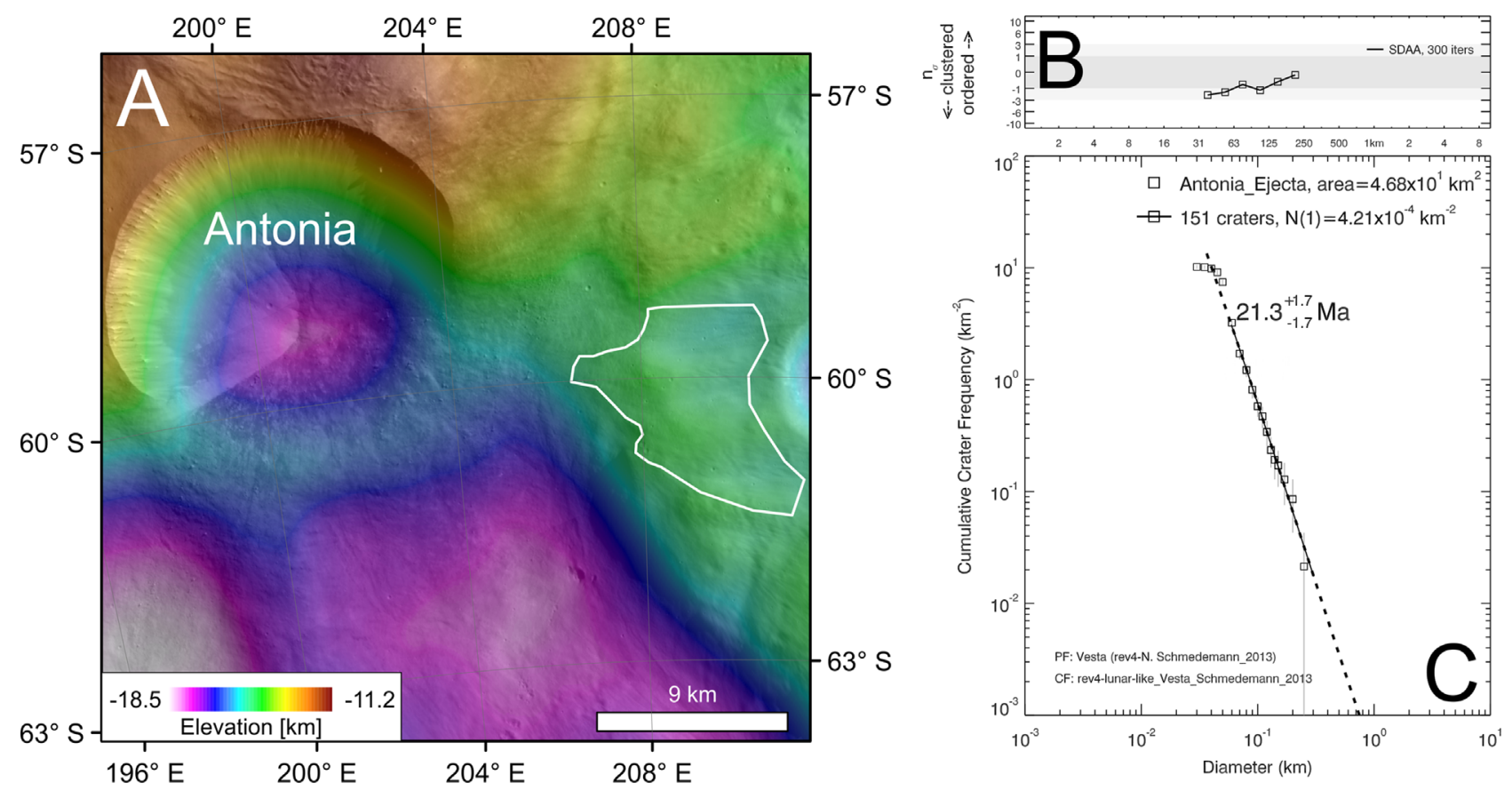

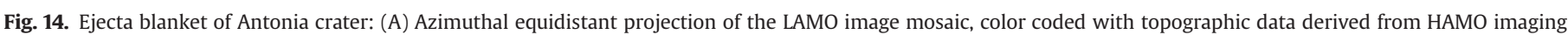
data. The counting area is given as a white solid outline. (B) Randomness analysis and (C) crater count of Antonia ejecta blanket - black squares.

Due to the shallow characteristics of O'Brien's chronology compared with the exponential slope of the lunar-like model chronology, we find increasingly different results for ages $>\sim 3 \mathrm{Ga}$. The most extreme examples are those areas which might give the lower limit of the formation age of the Veneneia basin. The lunar-like chronology give results in the range of about 3.7-3.8 Ga, while O'Brien's chronology gives model ages of about $4.5 \mathrm{Ga}$. Younger ages are relatively similar, because both chronologies are highly similar in their linear part from the present up to about $3 \mathrm{Ga}$.

Fig. 15 presents all measured ages and their respective locations on a global map of Vesta.

The oldest areas (heavily cratered terrains, North Pole area, ancient crater floor and Veneneia ejecta at Octavia) may show a crater distribution in equilibrium or quasi-equilibrium. Thus, for the measured crater sizes the derived age is a lower limit. If true, the formation age of Veneneia cannot be resolved and may also be older than $\sim 3.8 \mathrm{Ga}$. Only if very large craters and basins inside respectively larger areas are taken into account might higher crater retention ages be accessible. As mentioned in Section 2.3, if all large basins $\geq 100 \mathrm{~km}$ are taken into account and the corresponding surface area is the total area of Vesta, then the lunar-like chronology would provide a model age on the order of 3.9-4 Ga (SOM Section 3). In the heavily cratered terrain (HCT) area and in the North Pole area we find a resurfacing age of about 3.5 Ga. Similar ages can be found in the two LAMO measurements on top of the elevated terrain inside the ancient crater at $250^{\circ} \mathrm{E} /$ $30^{\circ} \mathrm{N}$ and in both HAMO measurements of the same region, the Rheasilvia ejecta blanket (oldest age at both areas of the Oppia ejecta) and as oldest age atop of the central peak of the Rheasilvia basin. On the Rheasilvia ejecta blanket and the basin's central peak ages occur in the range of 3.5-3.6 Ga that suggests the basin formed at about that time. Occurrences of similar ages in the northern hemisphere imply a major resurfacing event at that time over large areas. Such resurfacing could have its source in a tectonic response caused by the Rheasilvia impact. Hydrocode modeling of the Rheasilvia basin formation support the idea of global seismic effects from the Rheasilvia impact (Bowling et al.,
2013; Ivanov and Melosh, 2013). According to our results ages younger than $3.5 \mathrm{Ga}$ indicate events after the Rheasilvia formation. Thus, wide areas of the Rheasilvia basin floor do not reflect the basin formation age but later resurfacing events. While the Antonia ejecta blanketing ( $\sim 21 \mathrm{Ma}$ ) is only one example of many more of comparatively recent and local resurfacing events (e.g. Kneissl et al., 2014), there might be a number of older events that resurfaced large fractions of the Rheasilvia basin too. Close to or inside the Rheasilvia basin we find several ages clustering between $\sim 1.5$ and $\sim 2 \mathrm{Ga}$. It is not clear whether these events are truly separated in time or we are seeing one large event that resurfaced most of the Rheasilvia interior.

\section{Comparison of vestan crater retention ages with radiometric ${ }^{39} \mathrm{Ar}-{ }^{40} \mathrm{Ar}$ reset Ages of HED meteorites}

Radiometric ages of HED meteorites have been reported in a number of publications (e.g. Bogard, 1995, 2011; Bogard and Garrison, 2003). It has also been suggested that not all HED meteorites come from Vesta but that some HEDs may have their origin on probably five other Vesta-like parent bodies (Scott et al., 2009). These authors state that most of the anomalous eucrites show ages in excess of $4.45 \mathrm{Ga}$. The derivation of crater retention ages contains several different sources of systematic (modelbased) and stochastic (measurement-based) errors. The same applies to the independently working determination of radiometric ages with different sources of error (e.g. Bogard and Garrison, 2003). Despite the various sources of error it appears reasonable to compare the results of those independent techniques with each other in a similar representation, because there is the possibility that both techniques are able to record the same large impact events on Vesta. Bogard (2011) present a summed probability curve of 46 radiometric $\mathrm{Ar}-\mathrm{Ar}$ ages of HED meteorites. We use his plot and add probability curves of each measured crater retention age. We also plot summed curves derived from the individual curves of our measurements. All probability curves are given for both cases the lunar-like and O'Brien's chronology model. 
Table 6

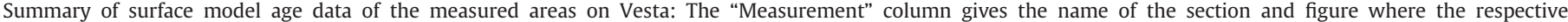

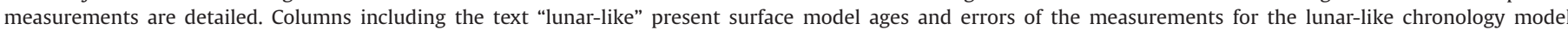

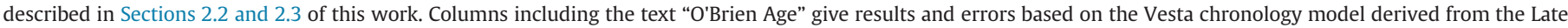

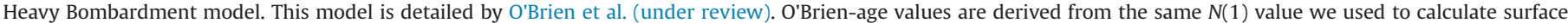

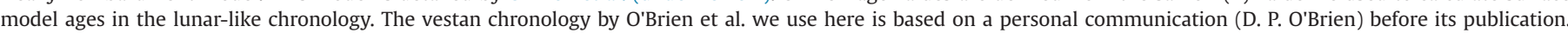
Thus, it may differ from the final version.

\begin{tabular}{|c|c|c|c|c|c|c|}
\hline Measurement & $\begin{array}{l}\text { Lunar-like } \\
\text { age [Ga] }\end{array}$ & $\begin{array}{l}\text { Lunar-like age “+” } \\
\text { Error [Ga] }\end{array}$ & $\begin{array}{l}\text { Lunar-like age “-” } \\
\text { Error [Ga] }\end{array}$ & $\begin{array}{l}\text { O'Brien } \\
\text { age [Ga] }\end{array}$ & $\begin{array}{l}\text { O'Brien age “+” } \\
\text { Error [Ga] }\end{array}$ & $\begin{array}{l}\text { O'Brien age “-" } \\
\text { Error [Ga] }\end{array}$ \\
\hline 3.2.1 Rheasilvia basin (Fig.5) & 1.77 & 0.21 & 0.21 & 1.72 & 0.2003 & 0.2017 \\
\hline $\begin{array}{l}\text { 3.2.2 Top of central peak of Rheasilvia base age } \\
\text { (Fig.6) }\end{array}$ & 3.51 & 0.077 & 0.16 & 4.37 & 0.1031 & 0.5627 \\
\hline $\begin{array}{l}\text { 3.2.2 Top of central peak of Rheasilvia } 1 . \\
\text { resurfacing (Fig.6) }\end{array}$ & 1.47 & 0.27 & 0.27 & 1.43 & 0.2526 & 0.2547 \\
\hline $\begin{array}{l}\text { 3.2.2 Top of central peak of Rheasilvia } 2 . \\
\text { Resurfacing (Fig.6) }\end{array}$ & 0.195 & 0.016 & 0.016 & 0.193 & 0.0158 & 0.0158 \\
\hline $\begin{array}{l}\text { 3.2.3 The Tuccia quadrangle - ridge and groove } \\
\text { terrain (Fig.7) }\end{array}$ & 1.85 & 0.22 & 0.22 & 1.79 & 0.2059 & 0.2074 \\
\hline 3.2.4 Floor of the Veneneia basin base age (Fig.8) & 3.13 & 0.2 & 0.53 & 3.13 & 0.5773 & 0.6264 \\
\hline $\begin{array}{l}\text { 3.2.4 Floor of the Veneneia basin 1. Resurfacing } \\
\text { (Fig.8) }\end{array}$ & 1.99 & 0.18 & 0.18 & 1.93 & 0.1647 & 0.1658 \\
\hline $\begin{array}{l}\text { 3.2.5 Heavily cratered terrain North of the equator } \\
\text { base age (Fig.9) }\end{array}$ & 3.75 & 0.071 & 0.15 & 4.52 & 0.0151 & 0.0423 \\
\hline $\begin{array}{l}\text { 3.2.5 Heavily cratered terrain North of the equator } \\
\text { 1. Resurfacing (Fig.9) }\end{array}$ & 3.5 & 0.046 & 0.065 & 4.32 & 0.1171 & 0.2118 \\
\hline 3.2.6 North pole area base age (Fig.10) & 3.75 & 0.04 & 0.055 & 4.52 & 0.0085 & 0.0129 \\
\hline 3.2.6 North pole area 1. Resurfacing (Fig.10) & 3.54 & 0.031 & 0.039 & 4.43 & 0.0311 & 0.1181 \\
\hline 3.2.7 Ancient crater at $250^{\circ} \mathrm{E} / 30^{\circ} \mathrm{N}-$ Floor (Fig.11) & 3.75 & 0.045 & 0.066 & 4.52 & 0.0097 & 0.016 \\
\hline 3.2.7 Ancient crater at $250^{\circ} \mathrm{E} / 30^{\circ} \mathrm{N}-$ ETN (Fig.11) & 3.55 & 0.089 & 0.23 & 4.44 & 0.0498 & 0.8097 \\
\hline 3.2.7 Ancient crater at $250^{\circ} \mathrm{E} / 30^{\circ} \mathrm{N}-$ ETS (Fig.11) & 3.58 & 0.037 & 0.05 & 4.47 & 0.0169 & 0.0485 \\
\hline $\begin{array}{l}\text { 3.2.7 Ancient crater at } 250^{\circ} \mathrm{E} / 30^{\circ} \mathrm{N} \text { - Floor-HAMO } \\
\text { (Fig.11) }\end{array}$ & 3.6 & 0.049 & 0.073 & 4.48 & 0.0179 & 0.0669 \\
\hline $\begin{array}{l}\text { 3.2.7 Ancient crater at } 250^{\circ} \mathrm{E} / 30^{\circ} \mathrm{N} \text { - Elevated } \\
\text { Terrain-HAMO (Fig.11) }\end{array}$ & 3.58 & 0.044 & 0.062 & 4.47 & 0.0186 & 0.0692 \\
\hline $\begin{array}{l}\text { 3.2.8 Oppia ejecta blanket northern part base age } \\
\text { (Fig.12) }\end{array}$ & 3.56 & 0.063 & 0.11 & 4.45 & 0.0319 & 0.3005 \\
\hline $\begin{array}{l}\text { 3.2.8 Oppia ejecta blanket northern part } 1 . \\
\text { Resurfacing (Fig.12) }\end{array}$ & 0.316 & 0.024 & 0.024 & 0.312 & 0.0232 & 0.0232 \\
\hline $\begin{array}{l}\text { 3.2.8 Oppia ejecta blanket southern part base age } \\
\text { (Fig.12) }\end{array}$ & 3.62 & 0.055 & 0.087 & 4.49 & 0.0173 & 0.0623 \\
\hline $\begin{array}{l}\text { 3.2.8 Oppia ejecta blanket southern part } 1 . \\
\text { Resurfacing (Fig.12) }\end{array}$ & 2.03 & 0.31 & 0.31 & 1.96 & 0.2886 & 0.2919 \\
\hline $\begin{array}{l}\text { 3.2.8 Oppia ejecta blanket southern part } 2 . \\
\text { Resurfacing (Fig.12) }\end{array}$ & 0.318 & 0.011 & 0.011 & 0.314 & 0.0104 & 0.0104 \\
\hline $\begin{array}{l}\text { 3.2.9 Octavia ejecta blanket south-east base age } \\
\text { (Fig.13) }\end{array}$ & 3.72 & 0.056 & 0.091 & 4.51 & 0.0125 & 0.0256 \\
\hline $\begin{array}{l}\text { 3.2.9 Octavia ejecta blanket south-east } 1 . \\
\text { Resurfacing (Fig.13) }\end{array}$ & 0.389 & 0.028 & 0.028 & 0.384 & 0.0280 & 0.0280 \\
\hline $\begin{array}{l}\text { 3.2.9 Octavia ejecta blanket south base age } \\
\text { (Fig.13) }\end{array}$ & 1.33 & 0.4 & 0.4 & 1.30 & 0.3795 & 0.3838 \\
\hline $\begin{array}{l}\text { 3.2.9 Octavia ejecta blanket south } 1 \text {. Resurfacing } \\
\text { (Fig.13) }\end{array}$ & 0.437 & 0.041 & 0.041 & 0.432 & 0.0405 & 0.0406 \\
\hline 3.2.10 Ejecta blanket of Antonia crater (Fig. 14) & 0.0213 & 0.0017 & 0.0017 & 0.0211 & 0.0017 & 0.0017 \\
\hline
\end{tabular}

We use the same time range Bogard (2011) used for his plot. The result is based on the age determination from the cumulative crater plots and is presented in Fig. 16. The shown probability curves represent the probability of any age from a given mean value of $N(1)$ and its symmetric gaussian error bars, which are projected onto the chronology function. In the non-linear parts of both chronology functions the model ages have asymmetric errors.

According to Bogard (2011), the radiometric ages in the range of 3.4-4.1 Ga are all derived from brecciated HED meteorites and presumably, represent shock-heating events from large impacts on the HED parent body. Bogard (2013) states “... quantitative Ar loss can occur in a $1 \mathrm{~km}$ half-thick slab at a temperature as low as $\sim 500 \mathrm{~K}$, whereas for a $10 \mathrm{~cm}$ half-thick slab a temperature of $\sim 1050 \mathrm{~K}$ would be required." $500 \mathrm{~K}$ is a value well in agreement with initial temperatures derived from recent hydrocode modeling for the floor of the Rheasilvia basin (Ivanov and Melosh, 2013). Also within hot ejecta blankets the $\mathrm{Ar}-\mathrm{Ar}$ chronometer can be reset as discussed for instance by Fernandes and Artemieva (2012).
The spectral characteristics of freshly exposed Rheasilvia ejecta (Le Corre et al., 2013), specific crater morphology and impact melt modeling (Williams et al., 2013) argue for high probability of impact melt occurrences on Vesta related to high energetic impacts. In addition, reset of the $\mathrm{Ar}-\mathrm{Ar}$ chronometer on meteorite parent bodies is not restricted to the dynamical situation in the early Solar System. It also happens in the current setup of the Solar System as illustrated by isotopic impact ages $<1.5 \mathrm{Ga}$ of chondrites (Bogard, 1995, 2011).

In both chronology models we see a prominent peak in the summed curves of the crater retention ages accompanied by a much smaller peak at slightly higher ages. The location of the prominent peak of both crater retention age models is intriguing. The O'Brien chronology suggests a high probability for a crater retention age close to a very prominent peak in the $\mathrm{Ar}-\mathrm{Ar}$ ages at $4.48 \mathrm{Ga}$. The corresponding measurements have been interpreted to show a relation to the formation of the Rheasilvia basin. Thus, it appears that the age of $\sim 4.48 \mathrm{Ga}$ for the vestan South Pole 


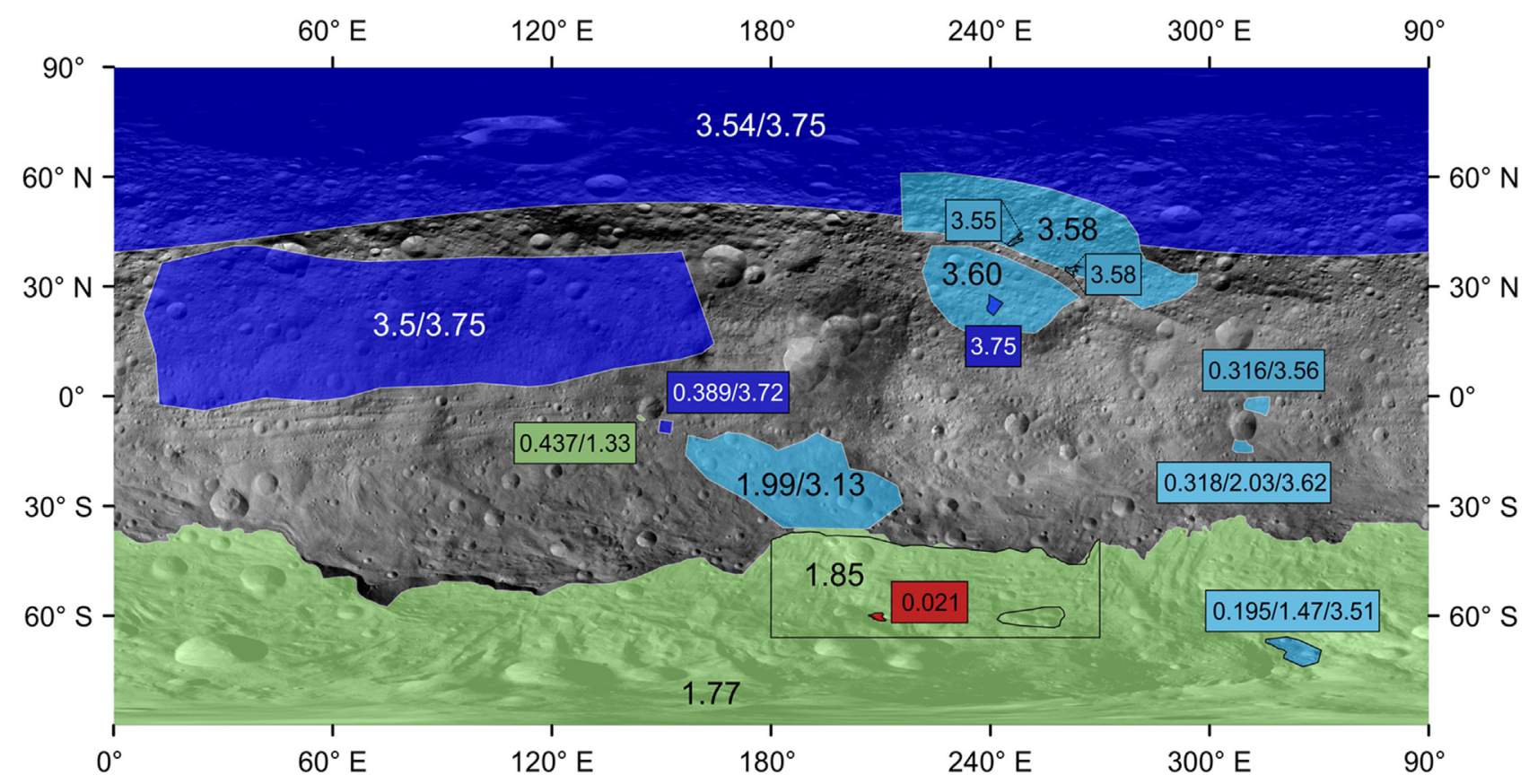

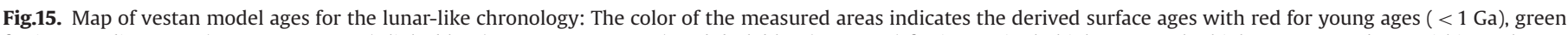

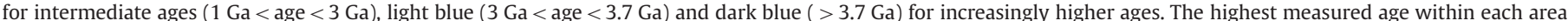

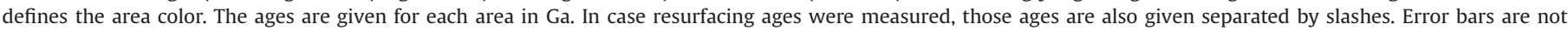

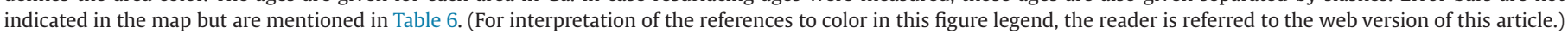

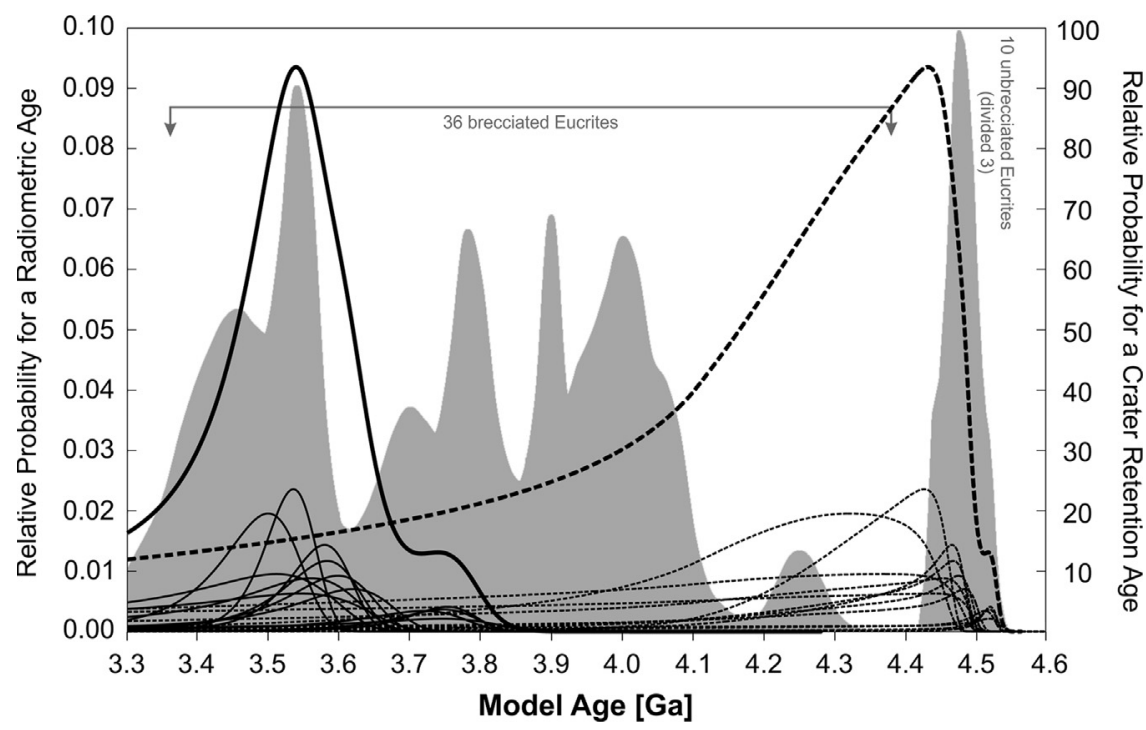

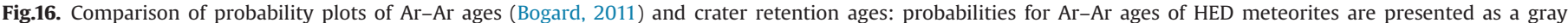

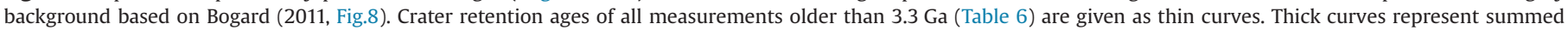
curves of the individual measurements. Solid curves - lunar-like chronology model, dashed curves - O'Brien chronology model.

depression suggested by Bogard and Garrison (2003) and Scott et al. (2009) is in agreement with crater retention ages of areas, which likely indicate the Rheasilvia basin formation age. The mentioned authors based their suggestion upon a hypothesis in order to explain why the narrow peak at $\sim 4.48 \mathrm{Ga}$ is defined by exclusively unbrecciated eucrites. The problem in their model is whether the eucrites could have escaped brecciation during the heavy bombardment in the early Solar System.

However, the majority of eucrites are brecciated as it is expected as a result of impact gardening. The $\mathrm{Ar}-\mathrm{Ar}$ chronometer of brecciated eucrites shows multiple large shock-heat events on Vesta that occurred basically in the time frame between 3.4 and $4.1 \mathrm{Ga}$ (Bogard, 2011). If Rheasilvia indeed formed $\sim 4.48 \mathrm{Ga}$ ago, it must have preserved a fresh morphology throughout this time of large impacts. Morphologically Rheasilvia is the youngest impact basin on Vesta. It is not superimposed by any other basin and its probable secondary craters cover large parts of the northern hemisphere and appear not to be superimposed by later basin forming events as well. Thus, Rheasilvia is expected to have formed towards the end of the time of large shock-heat events recorded in the brecciated eucrites 3.4-4.1 Ga ago. Indeed, for Rheasilvia formation related areas, the lunar-like chronology provides model ages with a high correlation to a shock-heat event recorded by the $\mathrm{Ar}-\mathrm{Ar}$ chronometer at $\sim 3.55 \mathrm{Ga}$.

The minor peak in the summed probability curve of the crater retention ages indicates an increased probability for a resurfacing event between 3.7 and $3.8 \mathrm{Ga}$ in the lunar-like model. In that time range the $\mathrm{Ar}-\mathrm{Ar}$ chronometer of HED meteorites recorded two 
shock-heat events. The respective minor peak of crater retention ages in the O'Brien chronology shows no corresponding peak in $\mathrm{Ar}-\mathrm{Ar}$ ages. The minor peak is derived from measurements, which probably indicate the lower limit of the formation age of the Veneneia basin. In case Rheasilvia formed only 200 to $300 \mathrm{Ma}$ after Veneneia, it might be possible that the $\mathrm{Ar}-\mathrm{Ar}$ chronometer of Veneneia impact heated material below the central peak of Veneneia remained open until it was excavated by the Rheasilvia impact. That requires large volumes of heated material from the Veneneia impact (e.g. Davison et al., 2010), Ar diffusion at relatively low temperatures and low cooling rates of only a few K/Ma within large volumes of heated material (Bogard, 2013). If true, probably a significant fraction of escaped material from the Rheasilvia impact could show the Rheasilvia impact age in the ArAr chronometer.

\section{Summary and discussion}

The purpose of this work was to adapt a reliable and well established technique on the Moon and Mars in order to derive surface model ages for geologic units on the Main Belt asteroids Gaspra, Ida, Lutetia and Vesta. We scaled the lunar production and chronology function (Neukum, 1984; Neukum and Ivanov, 1994) to the impact conditions of these asteroids and compare our results with either previous work, in the case of the three former asteroids or, in the case of Vesta, we compare the results of our lunar-like chronology with results of an alternative chronology (pers. comm. D. P. O'Brien) and the Ar-Ar age distribution of HED meteorites.

Production functions: There has been much discussion about the appropriate shape of a production function in order to fit measured crater distributions. On large bodies such as Mars or the Moon, small craters show a steep distribution on the order of -3 cumulative ( -4 differential). The questions remain: (i) does this distribution reflect the primary projectile distribution or is it contaminated by considerable amounts of distal non-clustered secondary craters (e.g. Shoemaker, 1965), and (ii) do resurfacing processes change the shape of the crater distribution resulting from just one prominent projectile distribution or are we dealing with multiple impactor populations with various slope characteristics changing early in solar system history and/or with the location in the solar system (Barlow and Strom, 1984; Neukum and Ivanov, 1994; Ivanov et al., 2002).

The low surface gravity on small asteroids such as Gaspra and Ida does not allow for the formation of secondary craters in large numbers because the secondary projectiles are easily lost to space. Thus, the observed steep crater distribution $(\sim-3 \mathrm{cum}$.) on Gaspra (Chapman et al., 1996b) is an indication that mainly primary projectiles are responsible for the steep crater distributions on the larger bodies and secondary craters only play a minor role (Neukum and Ivanov, 1994). Very early changes of the slope characteristic of the projectile distribution probably cannot be investigated on asteroidal surfaces at all, because the observable cratering record on even the largest bodies such as Vesta is limited to surface ages younger than $\sim 4 \mathrm{Ga}$ (lunar-like chronology) even for the largest and most durable impact structures.

The cratering record of Ida is close to an equilibrium distribution and no unambiguous results could be found for craters $<1 \mathrm{~km}$. If only fresh craters or craters $\geq 1 \mathrm{~km}$ are considered, we find a reasonable agreement with a lunar-like projectile distribution for Ida, which is similar to that observed on Gaspra. The larger asteroid Lutetia shows respectively larger craters too. Above about $1 \mathrm{~km}$ crater size, the lunar-like production function for Lutetia changes its slope. The measured crater distribution corresponds well to the production function, such that it also reflects the expected changes in slopes. Marchi et al. (2012b) did not count degraded craters and found a resurfacing-like kink in their crater distribution. They did not consider resurfacing effects as a reason but instead adjusted their crater production function by proposing a two layer model with different crater scaling behavior. We find the same kink if degraded craters are also excluded, but believe that the two-layer model is not required: the effect can be explained by crater degradation due to resurfacing processes.

Vesta shows a wide variety of crater size-frequency distributions, which indicate complex interactions of multiple processes. On relatively fresh surfaces we find a crater distribution in relatively good agreement with the derived vestan crater production function. However, we also find many crater distributions deficient in small craters. This effect is mostly interpreted as the result of resurfacing, where small craters are more easily obliterated than larger ones, for instance by gravity-driven mass wasting, ejecta blanketing or seismic shaking. On the other hand, in the narrow diameter range of $\sim 8$ $12 \mathrm{~km}$, we find a significantly steeper crater distribution than anticipated from the derived vestan production function. This phenomenon is primarily restricted to the northern vestan hemisphere. It is hypothesized that this is the result of Rheasilvia secondary cratering and/or Vestoid cratering.

We find good agreement with the conclusions of earlier work, that both Ida and Gaspra show a crater size-frequency distribution very similar to that of the lunar surface (Chapman et al., 1996a, 1996b; Ivanov et al., 2002). Furthermore, we also find the same similarity of the crater size-frequency distribution at the larger asteroids Lutetia and Vesta, extending the correspondence of the lunar crater size-frequency distribution to larger crater sizes and therefore larger projectile sizes. The responsible projectiles show a collisionally evolved characteristic very similar to that of the asteroid Main Belt. Among the investigated bodies only, Vesta is large enough to prove or disprove whether the impacting projectiles show an average Main Belt characteristic or a more pronounced deficiency in projectiles $\sim 25 \mathrm{~km}$ in diameter, typical for the inner and middle Main Belt (Tedesco et al., 2005; de Elía and Brunini, 2007). The respective crater size on Vesta corresponds to $\sim 140 \mathrm{~km}$. Craters of that size are mostly heavily degraded and the cratering record is incomplete, mainly due to the relatively late and large Rheasilvia impact. Thus, we do not see a clean cratering record in that size range and are unable to narrow down the source region of primary projectiles within the Main Belt without ambiguity based on our crater measurements.

Chronology functions and surface model ages: The chronology functions used for this work are based on an estimate of the current collision probabilities (Bottke et al., 1994). The lunar-like chronologies we use here are characterized by a linear correlation between surface age and cumulative crater frequency up to $\sim 3 \mathrm{Ga}$. It is not surprising that our measurements for fresh craters on the smaller asteroids Gaspra ( $270 \pm 0.07 \mathrm{Ma}$; resurfacing age) and Ida $(2.1 \pm 0.27 \mathrm{Ga}$; resurfacing age $)$ shows a general agreement with earlier work by other authors (Chapman et al., 1996a, 1996b). Those authors also assumed, as a first order approximation, a linear relationship between crater frequency and surface age and used an estimate of the current collision probabilities. Also the similarity of surface ages of Lutetia in our work and the result by Marchi et al. (2012b) is not a coincidence, because both groups used a lunar-like chronology scaled to the collision probability of Lutetia with other bodies. The differences to our approach are only the use of ArcGIS with the CraterTools plug-in for crater counting and a correction for projection errors caused by the deviations between the used reference body and the true surface topography. Good correlation to earlier results shows the reliability of our approach, which is then applied to the case of Vesta. Here however, we find significant deviation between our work and 
the work by Marchi et al. (2012c) and Schenk et al. (2012) with respect to the formation ages of the Veneneia and Rheasilvia basins. The differences are mostly based on the geologic interpretation of the used counting areas. Marchi et al. (2012c) and Schenk et al. (2012) use almost entirely fresh craters at the floor of both basins. For Rheasilvia, Marchi et al. (2012c) find $\sim 1 \mathrm{Ga}$ as the formation age, under the assumption that most of the obvious mass wasting occurred shortly after the basin formation. In contrast, after the crater relaxation movements have finished, we expect subsequent basin floor resurfacing, due to regolith slumping on slopes. This is a well-known phenomenon which may be triggered by subsequent impact cratering in the vicinity, and particularly when an impact occurs on the sloped terrain. For Veneneia, Schenk et al. (2012) states: "Crater counts also suggest a crater retention age for Veneneia of $2.1 \pm 0.2$ billion years (Fig. 4), predating Rheasilvia. Mantling of Veneneia by Rheasilvia ejecta may allow for an older age ..." ("Fig. 4" in their paper). The size and frequency of their measured craters in the range of roughly $10-17 \mathrm{~km}$ on the floor of Veneneia compares well to our measurement (Fig. 8), but we measured only fairly fresh craters, certainly younger than the Rheasilvia ejecta blanket. If the floor of the Veneneia basin was heavily modified by Rheasilvia's formation and they almost certainly measured craters younger than Rheasilvia ejecta, why do they still find an age $\sim 1$ Ga older than the proposed age for Rheasilvia? Both ages should be close unless the Rheasilvia ejecta blanket on the floor of Veneneia is older than the floor of Rheasilvia itself. Thus, we disagree with the interpretation of the formation ages for Rheasilvia and Veneneia given by Marchi et al. (2012c) and Schenk et al. (2012).

Our measurement of the eastern floor of Veneneia (Section 3.2.4) gives an age of $\sim 3.1 \mathrm{Ga}$, although our measured crater size-frequency distribution is very similar to that of Schenk et al. (2012) in the relevant size range. For the measured ages both chronologies are still in their linear sections and are very similar to each other since both groups based their individual chronologies on the current intrinsic collision probability of Vesta following Bottke et al. (1994). Thus, the differences in the model ages are mainly caused by different slopes of the production function. Where a given crater distribution is fitted at crater sizes $\geq 1 \mathrm{~km}$ (reference diameter for both chronologies), the steeper lunar-like distribution yields higher ages. If craters $<1 \mathrm{~km}$ are used for the fit the flatter model production function by Marchi et al. (2012c) yields higher ages. Whether a production function has the correct shape can be verified by cases where the same geologic unit/ event is dated similarly with a wide range of crater diameters. More comparisons between our lunar-like production function and the flatter model production function used by Marchi et al. (2012c) can be found in Kneissl et al. (2014).

It is difficult to find areas which give unambiguous crater data in order to determine the true formation ages of both prominent South Pole basins. Simply counting well defined craters on the basin floor, as has been done by Marchi et al. (2012c) and Schenk et al. (2012), does not account for the complex geologic processes acting in such areas over geologic timescales. Therefore, we tried to find areas in direct or indirect geologic relation to the formation ages of Veneneia and Rheasilvia which are not totally obscured by resurfacing processes. We determined 25 surface ages within 16 areas, which sometimes overlap in order to confirm results from various crater diameters or specifically to investigate cardinal points of basin morphology such as a central peak. We find several areas somehow connected to the formation of the Rheasilvia basin, which give results scattering in the range of roughly 3.5-3.6 Ga (cumulative fits). A summed probability curve from such measurements gives an age of $3.54{ }_{-0.09}^{+0.08} \mathrm{Ga}$ (errors derived from summation). Due to the global influence of the Rheasilvia impact as the last event of its size, the whole pre-Rheasilvia cratering record has been heavily obscured. Thus, it also erased evidence for the formation age of the Veneneia basin. We find some areas which still may indicate a formation age of Veneneia in the range of 3.73.8 Ga, but the respectively high crater frequencies may have been compromised by crater saturation already. A summed probability curve from the respective measurements gives an age of $3.74_{-0.08}^{+0.05} \mathrm{Ga}$. Due to the likely influence of cratering equilibrium/ saturation, the formation age of Veneneia is only a lower limit. Despite all the uncertainties, it is conceivable that Rheasilvia's formation could correspond to the youngest prominent peak in $\mathrm{Ar}-\mathrm{Ar}$ ages of brecciated HED meteorites (Bogard, 2011), leaving the minor peak in crater retention ages between 3.7 and $3.8 \mathrm{Ga}$ (Veneneia formation) to correspond to either a peak in $\mathrm{Ar}-\mathrm{Ar}$ ages at about $3.7 \mathrm{Ga}$ or $3.78 \mathrm{Ga}$. If we use the alternative chronology (pers. comm. D.P. O'Brien) with our measurements, we find a formation age of $4.43_{-0.28}^{+0.05}$ Ga for Rheasilvia and $4.52_{-0.02}^{+0.01}$ Ga for Veneneia. Given the relatively fresh appearance of the Rheasilvia basin, it appears somewhat unlikely that it survived for such a long time without being heavily modified by those large events recorded in the $\mathrm{Ar}-\mathrm{Ar}$ chronometer of brecciated eucrites in the time range of about 3.4-4.1 Ga (Bogard, 2011). For the purpose of comparison, large basins of all inner Solar System planets from more than $4.3 \mathrm{Ga}$ ago are also heavily degraded: for instance, the South-Pole Aitken basin on the lunar surface. Thus, it appears that results based on the alternative chronology for Vesta (O'Brien et al., under review) are inconsistent with observations in the inner Solar System. If we use the same comparison based on results of the lunar-like chronology for Vesta, we find a much better agreement with observations on the inner Solar System bodies.

In the literature, the proposed formation ages of the Rheasilvia basin range from $\leq 1 \mathrm{Ga}$ (Marzari et al., 1996) up to possibly 4.48 Ga (Bogard and Garrison, 2003; Bogard, 2011) with several estimates in the range of 3.5 and $3.8 \mathrm{Ga}$ (Bottke et al., 2005b; Moskovitz et al., 2008; Nesvorný et al., 2008; Roig et al., 2008; Neukum et al., 2011). Thus, our result for the age of Rheasilvia of about $3.5 \mathrm{Ga}$, which is derived from a lunar-like chronology, is well within previous expectations for the age of the vestan South Pole depression first observed in Hubble data. Based on results by Nesvorný et al. (2008) even the dynamical situation of the Vestoids is more consistent with our result for the age of Rheasilvia than an age as young as about $1 \mathrm{Ga}$.

If we compare the surface model age of Lutetia from all measured craters $\left(3.5_{-0.05}^{+0.04} \mathrm{Ga}\right)$, the surface model age of Ida from all measured craters $\left(3.6_{-0.1}^{+0.06} \mathrm{Ga}\right)$ and the surface model age of Gaspra from all measured craters $\left(2.9_{-1.1}^{+0.43} \mathrm{Ga}\right)$ with the youngest vestan impact basin Rheasilvia with $3.5_{-0.09}^{+0.08} \mathrm{Ga}$, the youngest lunar basin Orientale with $\sim 3.7$ Ga (Neukum et al., 2001; Fassett et al., 2012) and the youngest Martian basin Lyot with $\sim 3.4 \mathrm{Ga}$ (Werner, 2008), we find that only the tiny asteroid Gaspra is significantly younger than all other mentioned ages, which scatter within $~ 300 \mathrm{Ma}$. During that time span, the lunar chronology shows a transition from an exponential decay of impact rates towards a linear characteristic. It could be speculated that this transition marks the end of basin forming impacts on the larger bodies in the inner Solar System and in the asteroid Main Belt. The ages from Ida and Lutetia may also imply that around this time break-up events ceased, that could lead to basin-forming projectiles. This work will be extended in the future for instance with the youngest basin on Ceres, the next target of the Dawn mission, in order to understand what exactly changed in Solar System dynamics during the time frame of 3.4-3.7 Ga ago.

\section{Acknowledgments}

This work has been supported by the German Space Agency (DLR) on behalf of the Federal Ministry of Economics and 
Technology, grants 500W1101 (NS, TK, AN) and 50QM1301 (GM). We thank D.P. O'Brien for providing his preliminary solution for a vestan chronology based on the Late Heavy Bombardment model. We would like to thank the Dawn Flight Team for their work operating the spacecraft and returning the data used in this study. We also thank the Framing Camera team at the Max Planck Institute for Solar System Research and the German Aerospace Center (DLR) for processing the Dawn data and Sharon Ui for proofreading. We thank the anonymous reviewer and C. R. Chapman for their constructive comments.

\section{Appendix A. Supporting information}

Supplementary data associated with this article can be found in the online version at http://dx.doi.org/10.1016/j.pss.2014.04.004.

\section{References}

Alexander, C.M.O.D., Bowden, R., Fogel, M.L., Howard, K.T., Herd, C.D.K., Nittler, L.R., 2012. The provenances of asteroids, and their contributions to the volatile inventories of the terrestrial planets. Science.

Archinal, B.A., A'Hearn, M.F., Bowell, E., Conrad, A., Consolmagno, G.J., Courtin, R., Fukushima, T., Hestroffer, D., Hilton, J.L., Krasinsky, G.A., Neumann, G., Oberst, J., Seidelmann, P.K., Stooke, P., Tholen, D.J., Thomas, P.C., Williams, I.P., 2011. Report of the IAU working group on cartographic coordinates and rotational elements: 2009. Celestial Mech. Dyn. Astron. 109, 101-135.

Baldwin, R.B., 1964. Lunar crater counts. Astron. J. 69, 377.

Baldwin, R.B., 1974. Was there a "terminal lunar cataclysm" 3.9-4.0 × 109 years ago? Icarus 23, 157-166.

Baldwin, R.B., 2006. Was there ever a Terminal Lunar Cataclysm?: with lunar viscosity arguments. Icarus 184, 308-318.

Barlow, N.G., Strom, R.G., 1984. Martian crater size distributions and terrain age, NASA Technical Memorandum, NASA TM-86246, pp. 85-86.

Belton, M.J.S., Chapman, C.R., Klaasen, K.P., Harch, A.P., Thomas, P.C., Veverka, J., McEwen, A.S., Pappalardo, R.T., 1996. Galileo's encounter with 243 Ida: an overview of the imaging experiment. Icarus 120, 1-19.

Bierhaus, E.B., Dones, L., Alvarellos, J.L., Zahnle, K., 2012. The role of ejecta in the small crater populations on the mid-sized saturnian satellites. Icarus 218, 602-621.

Binzel, R.P., Xu, S., 1993. Chips off of asteroid 4 Vesta-evidence for the parent body of basaltic achondrite meteorites. Science0036-8075260 (5105), 186-191.

Bogard, D., 1995. Impact ages of meteorites: a synthesis. Meteoritics 30, 244

Bogard, D.D., 2011. K-Ar ages of meteorites: clues to parent-body thermal histories. Chem. der Erde-Geochem. 71 (3), 207-226.

Bogard, D.D., 2013. Analysis of thermal conditions required to reset $\mathrm{Ar}-\mathrm{Ar}$ ages. In: Proceedings of the Lunar and Planetary Institute Science Conference Abstracts, p. 1022.

Bogard, D.D., Garrison, D.H., 2003. ${ }^{39} \mathrm{Ar}-{ }^{40} \mathrm{Ar}$ ages of eucrites and thermal history of asteroid 4 Vesta. Meteorit. Planet. Sci. 38 (5), 669-710.

Bottke Jr., W.F., Morbidelli, A., Jedicke, R., Petit, J.-M., Levison, H.F., Michel, P., Metcalfe, T.S., 2002. Debiased orbital and absolute magnitude distribution of the near-earth objects. Icarus 156, 399-433.

Bottke, W.F., Durda, D.D., Nesvorny, D., Jedicke, R., Morbidelli, A., Vokrouhlicky, D., Levison, H., 2005a. The fossilized size distribution of the main asteroid belt. Icarus $175,111-140$.

Bottke, W.F., Durda, D.D., Nesvorný, D., Jedicke, R., Morbidelli, A, Vokrouhlicky, D., Levison, H.F., 2005b. Linking the collisional history of the main asteroid belt to its dynamical excitation and depletion [Erratum: 2006Icar..183..235B]. Icarus 179, 63-94.

Bottke Jr., W.F., Vokrouhlický, D., Rubincam, D.P., Nesvorný, D., 2006. The Yarkovsky and Yorp effects: implications for asteroid dynamics. Annu. Rev. Earth Planet. Sci. 34, 157-191.

Bottke, W.F., Nolan, M.C., Greenberg, R., Kolvoord, R.A., 1994. Velocity distributions among colliding asteroids. Icarus 107, 255-268.

Bottke, W.F., Vokrouhlicky, D., Chapman, C.R., Nesvorny, D., 2007. Gaspra's steep crater population was produced by a large recent breakup in the main asteroid belt. LPI Contribution no. 1338. In: Proceedings of the 38th Lunar and Planetary Science Conference, (Lunar and Planetary Science XXXVIII), March 12-16, 2007, League, Texas, p. 2165

Bottke, W.F., Vokrouhlický, D., Minton, D., Nesvorny, D., Morbidelli, A., Brasser, R., Simonson, B., Levison, H.F., 2012. An Archaean heavy bombardment from a destabilized extension of the asteroid belt. Nature 485 (78-81), 2012.

Bowling, T.J., Johnson, B.C., Melosh, H.J., Ivanov, B.A., O’Brien, D.P., Gaskell, R. Marchi, S., 2013. Antipodal terrains created by the Rheasilvia basin forming impact on asteroid 4 Vesta. J. Geophys. Res.: Planets 118, 1821-1834.

Britt, D.T., Yeomans, D., Housen, K., Consolmagno, G., 2002. Asteroid density, porosity, and structure. In: Bottke Jr., W.F., Cellino, A., Paolicchi, P., Binzel, R.P. (Eds.), Asteroids III. University of Arizona Press, Tucson, pp. 485-500.
Castillo-Rogez, J.C., Matson, D.L., Sotin, C., Johnson, T.V., Lunine, J.I., Thomas, P.C., 2007. Iapetus' geophysics: rotation rate, shape, and equatorial ridge. Icarus 190 179-202.

Chapman, C.R., Cohen, B.A., Grinspoon, D.H., 2007. What are the real constraints on the existence and magnitude of the late heavy bombardment? Icarus 189, 233-245.

Chapman, C.R., McKinnon, W.B., Matthews, M.S., 1986. Cratering of planetary satellites. In: Burns, J.A. (Ed.), Satellites, pp. 492-580.

Chapman, C.R., Ryan, E.V., Merline, W.J., Neukum, G., Wagner, R., Thomas, P.C. Veverka, J., Sullivan, R.J., 1996a. Cratering on Ida. Icarus 120, 77-86.

Chapman, C.R., Veverka, J., Belton, M.J.S., Neukum, G., Morrison, D., 1996b. Cratering on Gaspra. Icarus 120, 231-245.

Chesley, S.R., Ostro, S.J., Vokrouhlický, D., Čapek, D., Giorgini, J.D., Nolan, M.C., Margot, J.L., Hine, A.A., Benner, L.A.M., Chamberlin, A.B., 2003. Direct detection of the Yarkovsky effect by radar ranging to asteroid 6489 Golevka. Science 302, 1739-1742.

Cintala, M.J., Head, J.W., Veverka, J., 1978. Characteristics of the cratering process on small satellites and asteroids. In: Proceedings of the 9th Lunar and Planetary Science Conference, Houston, Texas, March 13-17, 1978. (A79-39253 16-91) Pergamon Press, Inc., New York, pp. 3803-3830.

Crater Analysis Techniques Working Group, Arvidson, R.E., Boyce, J., Chapman, C. Cintala, M., Fulchignoni, M., Moore, H., Neukum, G., Schultz, P., Soderblom, L., Strom, R., Woronow, A., Young, R., 1979. Standard techniques for presentation and analysis of crater size-frequency data. Icarus, 467-474.

Ćuk, M., Gladman, B.J., Stewart, S.T., 2010. Constraints on the source of lunar cataclysm impactors. Icarus 207, 590-594.

Daubar, I.J., McEwen, A.S., Byrne, S., Kennedy, M.R., Ivanov, B., 2013. The current martian cratering rate. Icarus 225, 506-516.

Davison, T.M., Collins, G.S., Ciesla, F.J., 2010. Numerical modeling of heating in porous planetesimal collisions. Icarus 208, 468-481.

de Elía, G.C., Brunini, A., 2007. Collisional and dynamical evolution of the main belt and NEA population. Astron. Astrophys. 466, 1159-1177.

Dohnanyi, J.S., 1971. Fragmentation and distribution of asteroids, NASA Special Publication 267 p. 263.

Farinella, P., Vokrouhlicky, D., Hartmann, W.K., 1998. Meteorite delivery via Yarkovsky orbital drift. Icarus 132, 378-387.

Fassett, C.I., Head, J.W., Kadish, S.J., Mazarico, E., Neumann, G.A., Smith, D.E., Zuber M.T., 2012. Lunar impact basins: stratigraphy, sequence and ages from superposed impact crater populations measured from Lunar Orbiter Laser Altimeter (LOLA) data. J. Geophys. Res. (Planets), 117.

Fernandes, V., Artemieva, N., 2012. Impact ejecta temperature profile on the Moon -what are the effects on the Ar-Ar dating method? In: Proceedings of the 43rd Lunar and Planetary Science Conference, held March 19-23, 2012, Woodlands, Texas. LPI Contribution no. 1659, id.1367.

Fernandes, V.A., Fritz, J., Weiss, B.P., Garrick-Bethell, I., Shuster, D.L., 2013. The bombardment history of the Moon as recorded by ${ }^{40} \mathrm{Ar}-{ }^{39} \mathrm{Ar}$ chronology. Meteorit. Planet. Sci., n/a-n/a.

Gladman, B.J., Davis, D.R., Neese, C., Jedicke, R., Williams, G., Kavelaars, J.J., Petit, J.-M., Scholl, H., Holman, M., Warrington, B., Esquerdo, G., Tricarico, P., 2009. On the asteroid belt's orbital and size distribution. Icarus 202, 104-118.

Gomes, R., Levison, H.F., Tsiganis, K., Morbidelli, A., 2005. Origin of the cataclysmic late heavy bombardment period of the terrestrial planets. Nature 435, 466-469.

Guillot, T., Gautier, D., 2007. 10.13-giant planets. In: Gerald, S. (Ed.), Treatise on Geophysics. Elsevier, Amsterdam, pp. 439-464.

Hartmann, W.K., 1975. Lunar "cataclysm”: a misconception? Icarus 24, 181-187.

Hartmann, W.K., 1984. Does crater "saturation equilibrium" occur in the solar system? Icarus 60, 56-74.

Hartmann, W.K., 2003. Megaregolith evolution and cratering cataclysm modelsLunar cataclysm as a misconception (28 years later). Meteorit. Planet. Sci. 38, 579-593.

Hartmann, W.K., Neukum, G., 2001. Cratering chronology and the evolution of Mars. Space Sci. Rev. 96, 165-194.

Hartmann, W.K., Werner, S.C., 2010. Martian Cratering 10. Progress in use of crater counts to interpret geological processes: examples from two debris aprons. Earth Planet. Sci. Lett. 294, 230-237.

Haskin, L.A., Korotev, R.L., Rockow, K.M., Jolliff, B.L., 1998. The case for an Imbrium origin of the Apollo Th-rich impact-melt breccias. Meteorit. Planet. Sci. 33 (5), 959-975.

Head, J.W., Fassett, C.I., Kadish, S.J., Smith, D.E., Zuber, M.T., Neumann, G.A Mazarico, E., 2010. Global distribution of large lunar craters: implications for resurfacing and impactor populations. Science 3291504.

Hiesinger, H., Head, J.W., Wolf, U., Jaumann, R., Neukum, G., 2002. Lunar mare basalt flow units: thicknesses determined from crater size-frequency distributions. Geophys. Res Lett. 29, 89-1-89-4 (CiteID 1248, DOI 1210).

Housen, K.R., Holsapple, K.A., 2011. Ejecta from impact craters. Icarus 211, 856-875. Housen, K.R., Holsapple, K.A., 2012. Craters without ejecta. Icarus 219, 297-306.

Inaba, S., Wetherill, G.W., Ikoma, M., 2003. Formation of gas giant planets: core accretion models with fragmentation and planetary envelope. Icarus 166, 46-62.

Ivanov, B., 2008. Size-Frequency Distribution Of Asteroids And Impact Craters: Estimates Of Impact Rate. In: Adushkin, V., Nemchinov, I. (Eds.), Catastrophic Events Caused by Cosmic Objects. Springer, Netherlands, pp. 91-116.

Ivanov, B.A., 2001. Mars/Moon cratering rate ratio estimates. Space Sci. Rev. 96 $87-104$.

Ivanov, B.A., 2006. Earth/Moon impact rate comparison: searching constraints for lunar secondary/primary cratering proportion. Icarus 183, 504-507.

Ivanov, B.A., Hartmann, W.K., 2007. 10.06-exogenic dynamics, cratering and surface ages. In: Gerald, S. (Ed.), Treatise on Geophysics. Elsevier, Amsterdam, pp. 207-242. 
Ivanov, B.A., Melosh, H.J., 2013. Two-dimensional numerical modeling of the Rheasilvia impact formation. J. Geophys. Res. (Planets) 118, 1545-1557.

Ivanov, B.A., Neukum, G., Bottke Jr., W.F., Hartmann, W.K., 2002. The comparison of size-frequency distributions of impact craters and asteroids and the planetary cratering rate. In: Bottke Jr., W.F., Cellino, A., Paolicchi, P., Binzel, R.P. (Eds.), Asteroids III. University of Arizona Press, Tucson, pp. 89-101.

Ivanov, B.A., Neukum, G., Wagner, R., 2001. Size-frequency distributions of planetary impact craters and asteroids. In: Marov, Mikhail Ya, Rickman, Hans (Eds.), Collisional processes in the solar system, 261. Kluwer Academic Publishers, Dordrecht (Astrophysics and space science library).

Jaumann, R., Williams, D.A., Buczkowski, D.L., Yingst, R.A., Preusker, F., Hiesinger, H. Schmedemann, N., Kneissl, T., Vincent, J.B., Blewett, D.T., Buratti, B.J., Carsenty, U., Denevi, B.W., De Sanctis, M.C., Garry, W.B., Keller, H.U., Kersten, E., Krohn, K. Li, J.Y., Marchi, S., Matz, K.D., McCord, T.B., McSween, H.Y., Mest, S.C., Mittlefehldt, D.W., Mottola, S., Nathues, A., Neukum, G., O'Brien, D.P., Pieters, C.M., Prettyman, T.H., Raymond, C.A., Roatsch, T., Russell, C.T., Schenk, P., Schmidt, B. E., Scholten, F., Stephan, K., Sykes, M.V., Tricarico, P., Wagner, R., Zuber, M.T. Sierks, H., 2012. Vesta's shape and morphology. Science 336, 687.

Jones, T.D., Lebofsky, L.A., Lewis, J.S., Marley, M.S., 1990. The composition and origin of the C, P, and D asteroids: water as a tracer of thermal evolution in the outer belt. Icarus $88,172-192$.

Kirchoff, M.R., Chapman, C.R., Marchi, S., Curtis, K.M., Enke, B., Bottke, W.F., 2013. Ages of large lunar impact craters and implications for bombardment during the Moon's middle age. Icarus 225, 325-341.

Kneissl, T., Schmedemann, N., Reddy, V., Williams, D.A., Walter, S., Neesemann, A. Michael, G., Jaumann, R., Krohn, K., Preusker, F., Roatsch, T., Le Corre, L., Nathues, A Hoffmann, M., Schäfer, M., Buczkowski, D., Garry, W.B., Yingst, R.A., Mest, S., Russell, C.T., Raymond, C.A., 2014. Morphology and formation ages of mid-sized postRheasilvia craters-geology of quadrangle Tuccia, Vesta. Icarus.

Kneissl, T., van Gasselt, S., Neukum, G., 2011. Map-projection-independent crater size-frequency determination in GIS environments-new software tool for ArcGIS. Planet. Space Sci. 59, 1243-1254.

Kreslavsky, M.A., 2007. Statistical characterization of spatial distribution of impact craters: implications to present-day cratering rate on mars. LPI Contributions $1353,3325$.

Le Corre, L., Reddy, V., Schmedemann, N., Becker, K.J., O'Brien, D.P., Yamashita, N., Peplowski, P.N., Prettyman, T.H., Li, J.-Y., Cloutis, E.A., Denevi, B.W., Kneissl, T., Palmer, E., Gaskell, R.W., Nathues, A., Gaffey, M.J., Mittlefehldt, D.W., Garry, W.B., Sierks, H., Russell, C.T., Raymond, C.A., De Sanctis, M.C., Ammanito, E., 2013. Olivine or impact melt: nature of the "Orange" material on Vesta from Dawn. Icarus 226, 1568-1594.

Le Feuvre, M., Wieczorek, M.A., 2011. Nonuniform cratering of the Moon and a revised crater chronology of the inner Solar System. Icarus 214, 1-20.

Lowry, S.C., Fitzsimmons, A., Pravec, P., Vokrouhlický, D., Boehnhardt, H., Taylor, P. A., Margot, J.-L., Galád, A., Irwin, M., Irwin, J., Kusnirák, P., 2007. Direct detection of the asteroidal YORP effect. Science 316, 272.

Mahaney, W.C., Kalm, V., Kapran, B., Hewitt, K., 2009. Clast fabric and mass wasting on Asteroid 25143-Itokawa: correlation with talus and other periglacial features on Earth. Sediment. Geol. 219, 44-57.

Malhotra, R., Strom, R.G., 2011. Comment on "Constraints on the source of lunar cataclysm impactors" (Cuk et al., 2010, Icarus 207, 590-594). Icarus 216, 359-362.

Malin, M.C., Edgett, K.S., Posiolova, L.V., McColley, S.M., Dobrea, E.Z.N., 2006. Present-day impact cratering rate and contemporary gully activity on Mars. Science 314, 1573-1577.

Marchi, S., Bottke, W.F., Kring, D.A., Morbidelli, A., 2012a. The onset of the lunar cataclysm as recorded in its ancient crater populations. Earth Planet. Sci. Lett. 325, 27-38.

Marchi, S., Massironi, M., Vincent, J.B., Morbidelli, A., Mottola, S., Marzari, F. Küppers, M., Besse, S., Thomas, N., Barbieri, C., Naletto, G., Sierks, H., 2012b. The cratering history of asteroid (21) Lutetia. Planet. Space Sci. 66, 87-95.

Marchi, S., McSween, H.Y., O'Brien, D.P., Schenk, P., De Sanctis, M.C., Gaskell, R. Jaumann, R., Mottola, S., Preusker, F., Raymond, C.A., Roatsch, T., Russell, C.T. 2012c. The violent collisional history of asteroid 4 Vesta. Science 336, 690.

Marchi, S., Mottola, S., Cremonese, G., Massironi, M., Martellato, E., 2009. A new chronology for the Moon and Mercury. Astron. J. 137, 4936-4948.

Marzari, F., Cellino, A., Davis, D.R., Farinella, P., Zappala, V., Vanzani, V., 1996. Origin and evolution of the Vesta asteroid family. Astron. Astrophys. 316, 248-262.

Massironi, M. Cremonese, G., Marchi, S., Martellato, E., Mottola, S., Wagner, R. 2009. Mercury's geochronology revised by applying model production function to Mariner 10 data: geological implications. Publication. Geophys. Res. Lett. 36 (21) (CiteID L21204).

McCord, T.B., Adams, J.B., Johnson, T.V., 1970. Asteroid Vesta: spectral reflectivity and compositional implications. Science 168, 1445-1447.

McEwen, A.S., Bierhaus, E.B., 2006. The importance of secondary cratering to age constraints on planetary surfaces. Annu. Rev. Earth Planet. Sci. 34, 535-567.

McSween, H., Mittlefehldt, D., Beck, A., Mayne, R., McCoy, T., 2011. HED meteorites and their relationship to the geology of Vesta and the Dawn Mission. Space Sci. Rev. 163, 141-174.

Michael, G.G., Neukum, G., 2010. Planetary surface dating from crater sizefrequency distribution measurements: Partial resurfacing events and statistica age uncertainty. Earth Planet. Sci. Lett. 294, 223-229.

Michael, G.G., Platz, T., Kneissl, T., Schmedemann, N., 2012. Planetary surface dating from crater size-frequency distribution measurements: spatial randomness and clustering. Icarus 218, 169-177.

Morbidelli, A., Levison, H.F., Tsiganis, K. Gomes, R., 2005. Chaotic capture of Jupiter's Trojan asteroids in the early solar system. Nature 435, 462-465.
Morbidelli, A., Marchi, S., Bottke, W.F., Kring, D.A., 2012. A sawtooth-like timeline for the first billion years of lunar bombardment. Earth Planet. Sci. Lett. 355, 144-151.

Morbidelli, A., Vokrouhlický, D., 2003. The Yarkovsky-driven origin of near-Earth asteroids. Icarus 163, 120-134

Moskovitz, N.A., Jedicke, R., Gaidos, E., Willman, M., Nesvorný, D., Fevig, R., Ivezić, Ž., 2008. The distribution of basaltic asteroids in the Main Belt. Icarus 198, 77-90.

Moskovitz, N.A., Willman, M., Burbine, T.H., Binzel, R.P., Bus, S.J., 2010. A spectroscopic comparison of HED meteorites and V-type asteroids in the inner Main Belt. Icarus 208, 773-788.

Nesvorný, D., Roig, F., Gladman, B., Lazzaro, D., Carruba, V., Mothé-Diniz, T., 2008. Fugitives from the Vesta family. Icarus 193, 85-95.

Neukum, G., 1984. Meteorite bombardment and dating of planetary surfaces. National Aeronautics and Space Administration, Thesis-February 1983, Washington, DC. Translated into English of "Meteoritenbombardement und Datierung Planetarer Oberflaechen” Munich, February 1983, pp. 1-186.

Neukum, G., Basilevsky, A.T., Kneissl, T., Chapmam, M.G., van Gasselt, S., Michael, G., Jaumann, R., Hoffmann, H., Lanz, J.K., 2010. The geologic evolution of Mars: episodicity of resurfacing events and ages from cratering analysis of image data and correlation with radiometric ages of Martian meteorites. Earth Planet. Sci. Lett. 294, 204-222.

Neukum, G., Basilevsky, A.T., Kneissl, T., Michael, G.G., Ivanov, B.A., 2012. On the history of early meteoritic bombardment of the Moon: did the lunar terminal cataclysm occur? Workshop on the Early Solar System Bombardment II, held 1-3 February 2012, in Houston, Texas. LPI Contribution no. 1649, pp. 55-56.

Neukum, G., Dietzel, H., 1971. On the development of the crater population on the moon with time under meteoroid and solar wind bombardment. Earth Planet. Sci. Lett. 12, 59-66.

Neukum, G., Hiller, K., 1981. Martian ages. J. Geophys. Res. 86, 3097-3121.

Neukum, G., Ivanov, B.A., 1994. Crater size distributions and impact probabilities on Earth from lunar, terrestrial-planet, and asteroid cratering data. In: Gehrels, Tom, Matthews, M.S., Schumann, A. (Eds.), Hazards due to Comets and Asteroids, Space Science Series, 1994. University of Arizona Press, Tucson, AZ, p. 359.

Neukum, G., Ivanov, B.A., Hartmann, W.K., 2001. Cratering records in the inner solar system in relation to the lunar reference system. Space Sci. Rev. 96, 55-86.

Neukum, G., Koenig, B., Arkani-Hamed, J., 1975. A study of lunar impact crater sizedistributions. The Moon 12, 201-229.

Neukum, G., Schenk, P., Schmedemann, N., Michael, G., Jaumann, R., Scully, J., Russell, C. T., O'Brien, D.P., Hiesinger, H., Nathues, A., Wagner, R., Marchi, S., 2011. Chronology and cratering at Vesta: first results from Dawn's survey orbit. EPSC-DPS Joint Meeting 2011, held 2-7 October, 2011 in Nantes, France. <A href= “〈http://meetings.copernicus.org/epsc-dps2011〉" > 〈http://meetings.copernicus.org/ epsc-dps2011 $\rangle<|\mathrm{A}\rangle, 501$.

Neukum, G., Wagner, R., Wolf, U., Denk, T., 2006. The cratering record and cratering chronologies of the Saturnian satellites and the origin of impac-tors: results from Cassini ISS data. European Planetary Science Congress, Berlin, Germany, 18-22 September 2006, pp. 610.

Nimmo, F., Korycansky, D.G., 2012. Impact-driven ice loss in outer solar system satellites: consequences for the late heavy bombardment. Icarus 219, 508-510.

O'Brien, D.P., Greenberg, R., 2005. The collisional and dynamical evolution of the main-belt and NEA size distributions. Icarus 178, 179-212.

O'Brien, D.P., Marchi, S., Schenk, P.M., Morbidelli, A., Bottke, W.F., et al., Constraining the cratering chronology of Vesta. Planetary and Space Sciences, under review.

Otto, K.A., Jaumann, R., Krohn, K., Matz, K.-D., Preusker, F., Roatsch, T., Schenk, P. Scholten, F., Stephan, K., Raymond, C.A., Russell, C.T., 2013. Mass-wasting features and processes in Vesta's south polar basin Rheasilvia. J. Geophys. Res.: Planets 118, 2279-2294.

Pätzold, M., Andert, T.P., Asmar, S.W., Anderson, J.D., Barriot, J-P., Bird, M.K. Häusler, B., Hahn, M., Tellmann, S., Sierks, H., Lamy, P., Weiss, B.P., 2011. Asteroid 21 Lutetia: low mass, high density. Science 334, 491-492.

Pike, R.J., 1980. Formation of complex impact craters: evidence from Mars and other planets. Icarus 43, 1-19.

Raymond, S.N., O'Brien, D.P., Morbidelli, A., Kaib, N.A., 2009. Building the terrestrial planets: constrained accretion in the inner Solar System. Icarus 203, 644-662.

Richardson, J.E., 2009. Cratering saturation and equilibrium: a new model looks at an old problem. Icarus 204, 697-715.

Richardson, J.E., 2013. Three-dimensional modeling of crater degradation via the effects of impact induced seismic shaking, with comparison to crater count data. LPI Contributions 1719, 2397.

Roatsch, T., Kersten, E., Matz, K.-D., Preusker, F., Scholten, F., Elgner, S., Jaumann, R., Raymond, C.A., Russell, C.T., 2013. High-resolution Vesta low altitude mapping orbit atlas derived from Dawn framing camera images. Planet. Space Sci. 85 293-298.

Roatsch, T., Kersten, E., Matz, K.D., Preusker, F., Scholten, F., Jaumann, R., Raymond, C.A., Russell, C.T., 2012. High resolution Vesta high altitude mapping orbit (HAMO) atlas derived from Dawn framing camera images. Planet. Space Sci. 73, $283-286$.

Roig, F., Nesvorný, D., Gil-Hutton, R., Lazzaro, D., 2008. V-type asteroids in the middle main belt. Icarus 194, 125-136.

Russell, C.T., Barucci, M.A., Binzel, R.P., Capria, M.T., Christensen, U., Coradini, A., De Sanctis, M.C., Feldman, W.C., Jaumann, R., Keller, H.U., Konopliv, A.S., McCord, T.B., McFadden, L.A., McKeegan, K.D., McSween, H.Y., Mottola, S. Nathues, A., Neukum, G., Pieters, C.M., Prettyman, T.H., Raymond, C.A., Sierks, H., Smith, D.E., Spohn, T., Sykes, M.V., Vilas, F., Zuber, M.T., 2007. Exploring the asteroid belt with ion propulsion: Dawn mission history, status and plans. Adv. Space Res. 40, 193-201. 
Russell, C.T., Raymond, C.A., 2011. The Dawn mission to Vesta and Ceres. Space Sci. Rev. 163, 3-23.

Russell, C.T., Raymond, C.A., Coradini, A., McSween, H.Y., Zuber, M.T., Nathues, A., De Sanctis, M.C., Jaumann, R., Konopliv, A.S., Preusker, F., Asmar, S.W., Park, R.S., Gaskell, R., Keller, H.U., Mottola, S., Roatsch, T., Scully, J.E.C., Smith, D.E., Tricarico, P., Toplis, M.J., Christensen, U.R., Feldman, W.C., Lawrence, D.J., McCoy, T.J., Prettyman, T.H., Reedy, R.C., Sykes, M.E., Titus, T.N., 2012. Dawn at Vesta: testing the protoplanetary paradigm. Science 336, 684.

Schenk, P., O'Brien, D.P., Marchi, S., Gaskell, R., Preusker, F., Roatsch, T., Jaumann, R. Buczkowski, D., McCord, T., McSween, H.Y., Williams, D., Yingst, A., Raymond, C., Russell, C., 2012. The geologically recent giant impact basins at Vesta's south pole. Science 336, 694.

Schmedemann, N., Kneissl, T., Michael, G., Neukum, G., Nathues, A., Sierks, H. Wagner, R., Krohn, K., Reddy, V., Hiesinger, H., Jaumann, R., Raymond, C.A., Russell, C.T., 2012. Crater size-frequency distribution (CSFD) and chronology of Vesta-crater counts matching HED ages. In: Proceedings of the 43rd Lunar and Planetary Science Conference, held March 19-23, 2012 at The Woodlands, Texas. LPI Contribution no. 1659, id.2544.

Scott, E.R.D., Greenwood, R.C., Franchi, I.A., Sanders, I.S., 2009. Oxygen isotopic constraints on the origin and parent bodies of eucrites, diogenites, and howardites. Geochim. Cosmochim. Acta 73 (19), 5835-5853.

Shoemaker, E.M., 1965. Preliminary analysis of the fine structure of the lunar surface in mare cognitum. The Nature of the Lunar Surface: Proceedings of the 1965 IAU-NASA Symposium held at Goddard Space Flight Center, April 15-16, 1965. Sponsored by IAU Commission 17 (the Moon) and NASA. Wilmot N. Hess, Donald H. Menz, John A. O'Keefe (Eds.). Library of Congress Catalog Card Number 65-27671; QB591.C748 1965 c.2., Johns Hopkins Press, Baltimore, MD, USA, 1965, pp. 23.

Spaute, D., Weidenschilling, S.J., Davis, D.R., Marzari, F., 1991. Accretional evolution of a planetesimal swarm: 1. A new simulation. Icarus 92, 147-164.

Strom, R.G., 1977. Origin and relative age of lunar and Mercurian intercrater plains. Phys. Earth Planet. Inter. 15, 156-172.

Strom, R.G., Malhotra, R., Ito, T., Yoshida, F., Kring, D.A., 2005. The origin of planetary impactors in the inner solar system. Science 309 (1847-1850), 2005.

Tedesco, E.F., Cellino, A., Zappal*, V., 2005. The statistical asteroid model. I. The Main-Belt population for diameters greater than $1 \mathrm{~km}$. Astron. J. 129, 2869-2886.

Tedesco, E.F., Veeder, G.J., Fowler, J.W., Chillemi, J.R., 1992. The IRAS Minor Planet Survey. Publication: the IRAS Minor Planet Survey. In: Tedesco, E.R., Veeder, G.J., Fowler, J.W., Chillemi, J.R. (Eds.), Philliips Laboratory, Hanscom Air Force Base, MA (December 1992. PL-TR-92-2049).

Tera, F., Papanastassiou, D.A., Wasserburg, G.J., 1974. Isotopic evidence for a terminal lunar cataclysm. Earth Planet. Sci. Lett. 22, 1-21.

Thomas, N., Barbieri, C., Keller, H.U., Lamy, P., Rickman, H., Rodrigo, R., Sierks, H., Wenzel, K.P., Cremonese, G., Jorda, L., Küppers, M., Marchi, S., Marzari, F. Massironi, M., Preusker, F., Scholten, F., Stephan, K., Barucci, M.A., Besse, S., ElMaarry, M.R., Fornasier, S., Groussin, O., Hviid, S.F., Koschny, D., Kührt, E., Martellato, E., Moissl, R., Snodgrass, C., Tubiana, C., Vincent, J.B., 2012. The geomorphology of (21) Lutetia: results from the OSIRIS imaging system onboard ESA's Rosetta spacecraft. Planet. Space Sci. 66, 96-124.
Thomas, P.C., Belton, M.J.S., Carcich, B., Chapman, C.R., Davies, M.E., Sullivan, R. Veverka, J., 1996. The shape of Ida. Icarus 120, 20-32.

Thomas, P.C., Binzel, R.P., Gaffey, M.J., Storrs, A.D., Wells, E.N., Zellner, B.H., 1997. Impact excavation on Asteroid 4 Vesta: hubble space telescope results. Science 277, 1492-1495.

Thomas, P.C., Veverka, J., Simonelli, D., Helfenstein, P., Carcich, B., Belton, M.J.S. Davies, M.E., Chapman, C., 1994. The shape of Gaspra. Icarus 107, 23-36.

Tsiganis, K., Gomes, R., Morbidelli, A., Levison, H.F., 2005. Origin of the orbita architecture of the giant planets of the Solar System. Nature 435, 459-461.

van der Bogert, C.H., Hiesinger, H., McEwen, A.S., Dundas, C., Bray, V., Robinson, M.S. Plescia, J.B., Reiss, D., Klemm, K., Lroc, T., 2010. Discrepancies between crater sizefrequency distributions on ejecta and impact melt pools at lunar craters: an effect of differing target properties? In: Proceedings of the 41st Lunar and Planetary Science Conference, held March 1-5, 2010 in The Woodlands, Texas. LPI Contribution no. 1533, p. 2165.

Vasavada, A.R., Bandfield, J.L., Greenhagen, B.T., Hayne, P.O., Siegler, M.A., Williams, J.-P., Paige, D.A., 2012. Lunar equatorial surface temperatures and regolith properties from the Diviner Lunar Radiometer experiment. J. Geophys. Res. Planets 117, E00H18.

Weidenschilling, S.J., 1974. A model for accretion of the terrestrial planets. Icarus 22, 426-435.

Weidenschilling, S.J., 1976. Accretion of the terrestrial planets II. Icarus 27, 161-170.

Weidenschilling, S.J., 1977. The distribution of mass in the planetary system and solar nebula. Astrophys. Space Sci. 51, 153-158.

Weidenschilling, S.J., 2011. Initial sizes of planetesimals and accretion of the asteroids. Icarus 214, 671-684.

Werner, S.C., 2005. Major Aspects of the Chronostratigraphy and Geologic Evolutionary History of Mars (Ph.D. thesis) p. 27.

Werner, S.C., 2008. The early martian evolution-constraints from basin formation ages. Icarus 195, 45-60.

Werner, S.C., Harris, A.W., Neukum, G., Ivanov, B.A., 2002. The near-Earth asteroid size-frequency distribution: a snapshot of the Lunar impactor size-frequency distribution. Icarus 156, 287-290.

Werner, S.C., Ivanov, B.A., Neukum, G., 2009. Theoretical analysis of secondary cratering on Mars and an image-based study on the Cerberus Plains. Icarus 200, 406-417.

Wetherill, G.W. 1975. Late heavy bombardment of the moon and terrestrial planets. In: Proceedings of the Lunar and Planetary Science Conference, pp. 1539-1561.

Wilhelms, D.E., McCauley, J.F., Trask, N.J., 1987. The geologic history of the moonWashington: U.S. G.P.O.; Denver, CO (Federal Center, Box 25425, Denver 80225): for sale by the books and open-file reports section. U.S. Geological Survey, 1987.

Williams, D.A., O’Brien, D.P., Schenk, P.M., Denevi, B.W., Carsenty, U., Marchi, S. Scully, J.E.C., Jaumann, R., De Sanctis, M.C., Palomba, E., Ammannito, E., Longobardo, A., Magni, G., Frigeri, A., Russell, C.T., Raymond, C.A., Davison, T. M., 2013. Lobate and flow-like features on asteroid Vesta. Planetary and Space Science, http://dx.doi.org/10.1016/j.pss.2013.06.017.

Zahnle, K., Schenk, P., Dones, L., Levison, H., 2003. Cratering rates in the outer solar system. In: Proceedings of the 34th Annual Lunar and Planetary Science Conference, March 17-21, 2003, League City, Texas. Abstract no. 1522. 